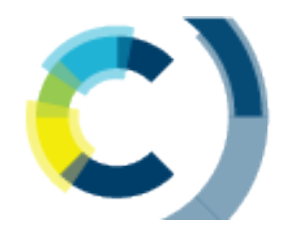

\title{
Green
}

\section{Successful Development of a Method for the Incorporation of Fmoc-Arg(Pbf)-OH in Solid-Phase Peptide Synthesis using N Butylpyrrolidinone (NBP) as a Green Solvent}

\begin{tabular}{|r|l|}
\hline Journal: & Green Chemistry \\
\hline Manuscript ID & Draft \\
\hline Article Type: & Paper \\
\hline Author: & n/a \\
\hline Complete List of Authors: & $\begin{array}{l}\text { Albericio, Fernando; University of KwaZulu-Natal, Chemistry \& Physics; } \\
\text { University of Barcelona, Organic Chemistry } \\
\text { de la Torre, Beatriz; University of KwaZulu-Natal College of Health } \\
\text { Sciences, } \\
\text { Kumar, Ashish; University of KwaZulu-Natal, Chemistry \& Physics } \\
\text { Alhassan, Mahama; University of KwaZulu-Natal, Chemistry \& Physics } \\
\text { Bucher, Christoph; Novartis Pharma AG }\end{array}$ \\
\hline
\end{tabular}




\section{Green Chemistry}

\section{ARTICLE}

\section{Successful Development of a Method for the Incorporation of Fmoc-Arg(Pbf)-OH in Solid-Phase Peptide Synthesis using N-Butylpyrrolidinone (NBP) as a Green Solvent}

Received 00th January 20xx, Accepted 00th January 20xx DOI: $10.1039 / x 0 x \times 00000 x$

\author{
Beatriz G. de la Torre, ${ }^{* a}$ Ashish Kumar, ${ }^{a, b}$ Mahama Alhassan, ${ }^{b}$ Christoph Bucher, ${ }^{,}$Fernando \\ Albericio, ${ }^{* b, d, e}$ John Lopez ${ }^{* c}$,
}

\begin{abstract}
NBP has proved an excellent alternative solvent to the hazardous DMF for SPPS. Here we studied the incorporation of Fmoc$\operatorname{Arg}(\mathrm{Pbf})-\mathrm{OH}$, one of the most problematic amino acids, into a growing peptide chain. The poor performance of this amino acid is attributed to the formation of a fully inactive $\delta$-lactam, which causes a reduction in yield and very often the concomitant formation the corresponding des-Arg peptides. This problem is exacerbated when NBP is used as solvent, presumably because of its high viscosity, which impairs the penetration of the coupling cocktail into the resin. To tackle this issue, we propose the following strategy for the safe introduction of Fmoc- $\mathrm{Arg}(\mathrm{Pbf})-\mathrm{OH}$ in SPPS at $45^{\circ} \mathrm{C}$, keeping excesses to a minimum: 1.75 equiv. of the protected amino acids, 1.8 equiv. of DIC, and 1.5 equiv. of OxymaPure. The cornerstone of the strategy is to carry out in situ activation. In this regard, Fmoc-Arg(Pbf)-OH and OxymaPure dissolved in NBP were added to peptidyl-resin, allow to reach the $45^{\circ} \mathrm{C}$, then half the DIC was added and left for $30 \mathrm{~min}$, followed by the other half and some extra Fmoc-Arg(Pbf)- $\mathrm{OH}$. During the entire process, the temperature was kept at $45^{\circ} \mathrm{C}$, with the double purpose of reducing the viscosity of NBP, thus facilitating the penetration of the coupling cocktail into the resin, and speeding up the coupling itself. It is envisaged that this strategy could be widely used to improve the performance of SPPS, including the industrial preparation of peptides using this approach.
\end{abstract}

\section{Introduction}

The number of peptide-based drugs approved by the two most important regulatory agencies, namely the Food and Drug Administration (FDA) and the European Medicines Agency (EMA), has risen notably in recent years. ${ }^{1-4}$ With respect to the validation of the role of the peptides in the drug arena, more important than the number of peptides on the market (more than $60^{1}$ ) is the number of peptides in clinical phases (more than $\left.170^{1}\right)$. From a structural point of view, peptides either on the market or in active development are characterized by increasing complexity. Thus, those containing more than 30 amino acids, several cycles, and/or pending fatty or polyethylenglycol chains are common in the various drug discovery phases. Indeed, this is exemplified by the peptides approved by the FDA in 2017, an exceptional year for the peptide harvest, with six peptide approvals. $^{5}$

There is no doubt that the solid-phase peptide synthesis (SPPS) strategy developed by the Nobel Laurate R. Bruce Merrifield has

${ }^{a}$ KwaZulu-Natal Research Innovation and Sequencing Platform (KRISP), School of Laboratory Medicine and Medical Sciences, College of Health Sciences, University of KwaZulu-Natal, Durban 4041, South Africa.

bPeptide Science Laboratory, School of Chemistry and Physics, University of KwaZulu-Natal, Durban 4001, South Africa.

'Novartis Pharma AG, Novartis Campu Campus, WSJ-145-5.51, CH-4056 Basel, Switzerland.

${ }^{d}$ CIBER-BBN, Networking Centre on Bioengineering, Biomaterials and Nanomedicine, Barcelona Science Park, University of Barcelona, 08028 Barcelona, Spain.

e Department of Organic Chemistry, University of Barcelona, 08028 Barcelona, Spain. been key for peptides to be considered alternative drugs to the so-called small molecules. ${ }^{6}$ Although in its early days SPPS was heavily criticized by Merrifield's colleagues, ${ }^{7}$ the power of this approach has been widely exploited, first as a potent research tool and later as the strategy of choice for the production of most of the peptides on the market. ${ }^{8,} 9$ In this regard, the establishment of the multi kilogram-scale production of enfuvirtide (T-20 or Fuzeon), a first-in-class membrane fusion inhibitor for the treatment of HIV, is considered the validation of SPPS for the production of peptides. ${ }^{10}$ Enfuvirtide is a 36amino acid peptide that was synthesized by a hybrid solid phase-solution strategy, in which three protected peptides were prepared in solid phase and then assembled in solution. Although this compound did not become the expected commercial blockbuster, its development marked a breakthrough. At that time, it became clear that almost any peptide could be synthetically affordable, mostly using SPPS. As a minor, but important, consequence of the bulk synthesis of enfuvirtide was that the high consumption of protected amino acids and chlorotrityl chloride (CTC) resin-the solid support used for the preparation of the protected fragments-leaded the cost of these materials fell, thereby bringing about a certain democratization of the peptide field.

Interesting, the most expensive protected amino acid currently on the market, Fmoc-Arg(Pbf)-OH, was one of the exceptions in the enfuvirtide sequence. However, to be fully honest, it is also important to clarify that the synthesis of Fmoc- $\mathrm{Arg}(\mathrm{Pbf})-\mathrm{OH}$ is probably the most complex of all common protected amino acids. ${ }^{11}$ 
From a sustainability perspective, SPPS has a number of pros and cons. The pros include the following: (i) easy manipulations, as all reactions take place in the same reactor, and therefore no isolation of intermediates is needed and no major cleaning procedures are required; (ii) easy work-up, because the excess of reagents and soluble side-products are removed by filtration and washings, thereby allowing the use of an excess of reagents, thereby facilitating high conversion yields; (iii) use of solvents of high boiling point; and (iv) possibility of partial and even full automatization. On the other hand, the cons encompass the following: (i) the same use of reagent excess, although this is mitigated in large scale production, where only slight excesses of the reagents are used; (ii) very poor atom economy [e.g., 0.24 for Fmoc-Arg(Pbf)-OH and 0.19 for Fmoc-Gly-OH]; and (iii) the large volume of solvents used for washing the resin after each synthetic step.

In this last regard, the large consumption of solvents and the fact that $\mathrm{N}, \mathrm{N}$-dimethylformamide (DMF) has been by far the most widely used solvent in SPPS are the main drawbacks in greening the strategy. DMF is reprotoxic and, like other polar aprotic solvents, such as N-methylpyrrolidone (NMP), and dimethylacetamide (DMA), has been labeled by REACH-ECHA as a Substance of Very High Concern (SVHC) and is therefore a focus for replacement. To tackle this issue, several pharmaceutical industries participating in the ACS Green Chemistry Institute Pharmaceutical Roundtable have shown interest in identifying green solvents for SPPS. ${ }^{12}$ In recent years, our groups have been working independently to identify an alternative solvent to DMF. In this regard, one group has developed strategies in which 2-methyltetrahydrofurane (MeTHF) and $\gamma$-valerolactone (GVL) have been shown to be suitable alternatives to DMF. ${ }^{13-16}$ The other group has proposed the use of $\mathrm{N}$-butylpyrrolidone (NBP) for the same purpose. ${ }^{17} \mathrm{NBP}$ is a polar aprotic solvent with a high boiling point $\left(243^{\circ} \mathrm{C}\right)$. According to OECD test methods, NBP is non-mutagenic, nonreprotoxic and biodegradable and is therefore friendlier than other amide-based solvents of related structure, such as DMF and NMP.

In our previous work, ${ }^{17}$ and after a careful evaluation of 34 nonhazardous solvents, NBP was shown to outperform the other solvents tested in terms of resin swelling, Fmoc removal efficiency, and coupling yield and thus emerges as a strong candidate to replace DMF. NBP was successfully applied for the semi-industrial synthesis of the linear precursor of the octapeptide Octreotide. Although the purity of the crude peptide synthesized with NBP was slightly inferior to that prepared with DMF ( $80 \%$ vs. $86 \%)$, the impurity profile was similar in both cases. This observation reinforces the notion of the appropriateness of NBP to achieve greener SPPS.

Here, in an attempt to generalize the use of NBP, we studied the compatibility of this solvent with the coupling of Fmoc-Arg(Pbf)$\mathrm{OH}$. Arg is an important residue and has been object of several methodological studies. ${ }^{18}$ The relevance of this amino acid lies in its presence in many peptide-based APIs, such as Etelcalcetide, Abaloparatide, and Semaglutide, among others approved by the FDA in recent years. ${ }^{5}$ Arg is also key for the development of the so-called Cell-Penetrating Peptides
(CPPs). ${ }^{19}$ It is important to note that Fmoc-Arg(Pbf)-OH, which is the most used building block for the introduction of Arg into SPPS, is the most expensive of all protected proteinogenic amino acids and, like all Arg derivatives, it undergoes an important side-reaction upon activation. In this regard, once the carboxylic group of the protected Arg is activated, $\delta$-lactam formation takes place, with the corresponding formation of a six-member ring (Scheme 1), which blocks the incorporation of Arg into the growing peptide sequence. Although this sidereaction does not modify the final peptide, it often leads to the formation of deletion peptides, in which Arg is missing. To mitigate this, repetitive couplings of protected Arg are carried out, a procedure that increases the cost of the whole process. This drawback is exacerbated during the industrial preparation of Arg-containing peptides, where minimum excesses are used and repetitive couplings have a penalty in time, solvent consumption, and cost.

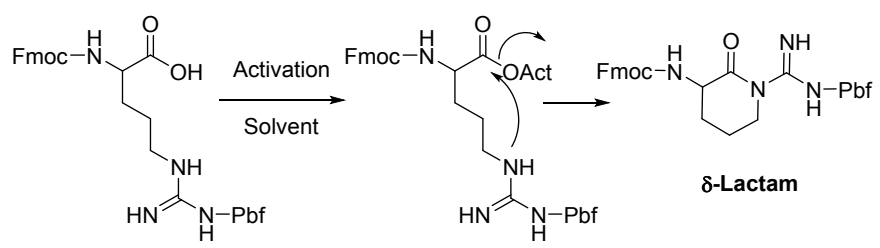

Scheme 1. Activation of Fmoc- $\mathrm{Arg}(\mathrm{Pbf})-\mathrm{OH}$ with the concomitant formation of $\delta$-lactam.

\section{Results and discussion}

The present study was carried using DIC and OxymaPure as coupling cocktail in DMF and NBP as solvents. OxymaPure ${ }^{20}$ is the additive of choice for the most peptide syntheses and has been proposed by the ACS Green Chemistry Institute Pharmaceutical Roundtable as the greenest aditive. ${ }^{12}$

To study the activation kinetics of $\mathrm{Fmoc}-\mathrm{Arg}(\mathrm{Pbf})-\mathrm{OH}$ and the subsequent ?-lactam formation, an equimolar mixture of the three reagents, Fmoc-Arg(Pbf)-OH, DIC, and OxymaPure, was prepared in the two solvents, DMF and NBP, at room temperature (RT). In the absence of any nucleophile, the behavior observed in both solvents was very similar (Figure 1A). The activation in DMF was slightly faster than in NBP, as was ?-lactam formation. When the same experiment was carried out at $60^{\circ} \mathrm{C}$ (Figure 1B), the activation and ?-lactam formation were much faster than at RT, as expected. Furthermore, the previous tendency was inversed and although both solvents gave similar results, the complete conversion of $\mathrm{Fmoc}-\mathrm{Arg}(\mathrm{Pbf})-\mathrm{OH}$ into its $\delta$-lactam was slightly faster in NBP.

Next, the previous experiment at RT was repeated in the presence of a nucleophile, the peptide H-Gly-Phe-Leu anchored to a Rink amide polystyrene resin. This reaction mixture allowed us to study the competition between coupling and lactam formation by the HPLC analysis of the supernatant and the composition of the peptide after cleavage from the resin (Scheme 2). 


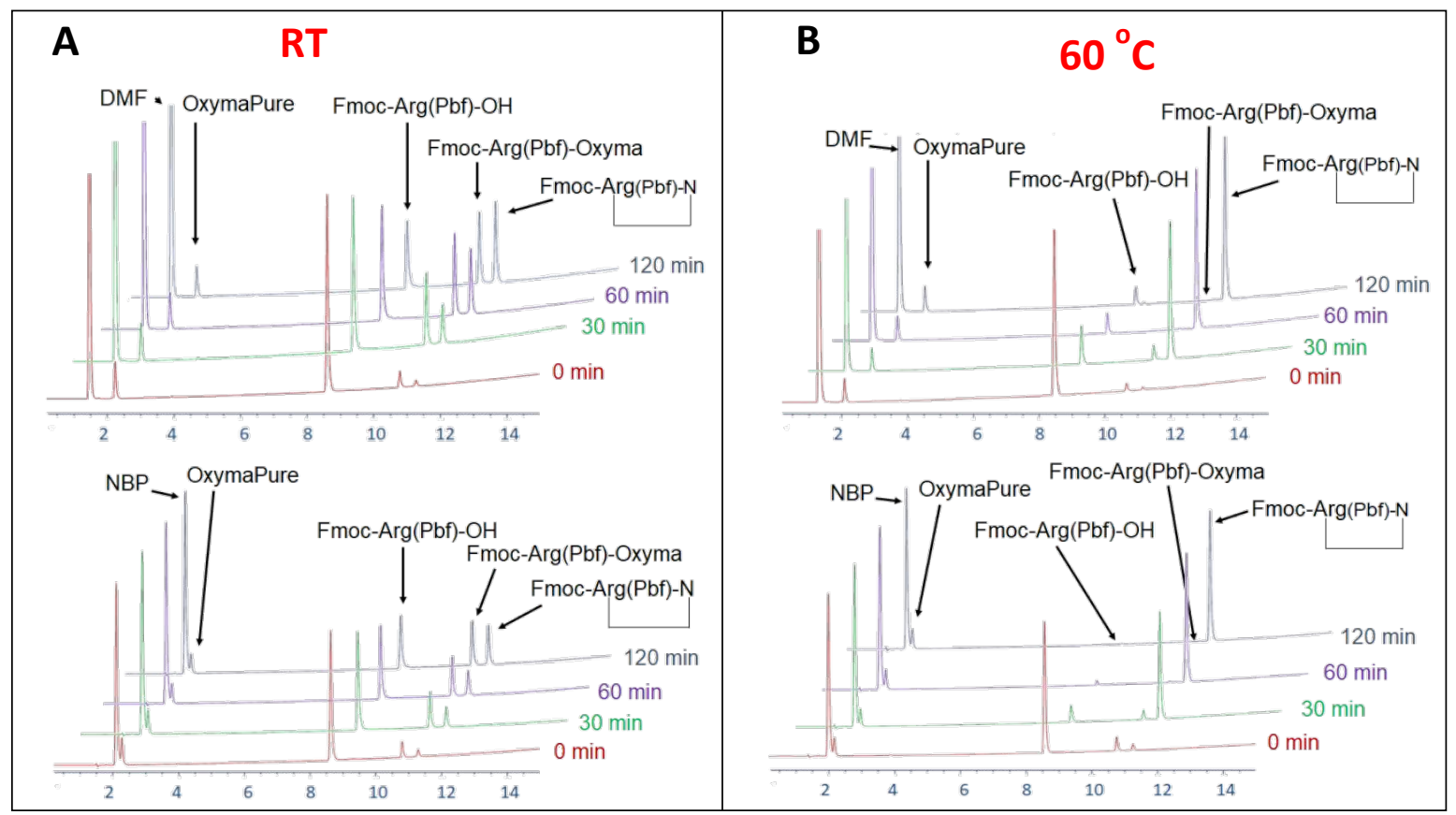

Figure 1. HPLC analysis of the activation kinetics and ? -lactamization of Fmoc-Arg(Pbf)-OH with DIC and OxymaPure in DMF and NBP at A) RT and B) $60^{\circ} \mathrm{C}$ (see methods section for HPLC conditions). Note: The peak corresponding to Fmoc-Arg(Pbf)-OH could be due to unreacted amino acid or mostly to instability of the activated species [Fmoc-Arg(Pbf)-Oxyma] under aqueous HPLC conditions.

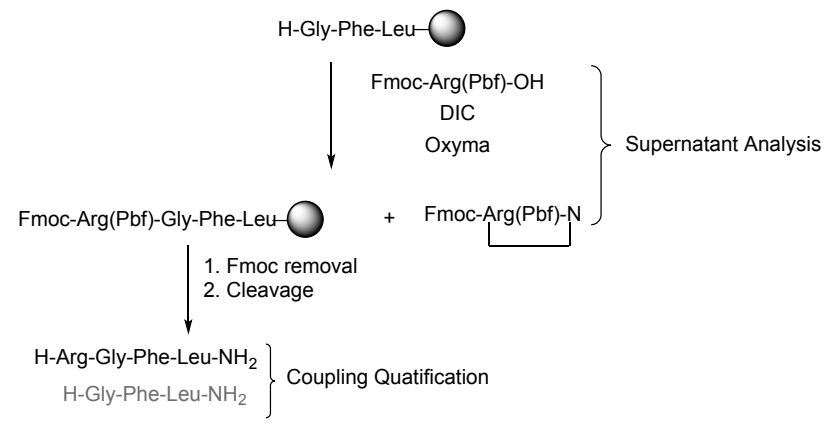

Scheme 2. Reaction of the activated Fmoc-Arg(Pbf)-OH with a tripeptidyl resin.

For these sets of experiments, Fmoc-Arg(Pbf)-OH and OxymaPure were dissolved in the corresponding solvent, and DIC was added at a ratio of $1: 1: 1$ at a concentration $0.075 \mathrm{M}$. Next, 1.5 equiv. of the mixture was added to the resin, which had been previously washed with the solvent, which was removed by filtration. HPLC analysis of the supernatant showed almost negligible formation of ?-lactam when DMF was used, in comparison with NBP (Figure 2). In contrast, analysis of the cleaved peptide showed more than $98 \%$ incorporation of $\operatorname{Arg}(\mathrm{Pbf})$ when DMF was used, but less of $50 \%$ in the case of NBP (Figure 2).
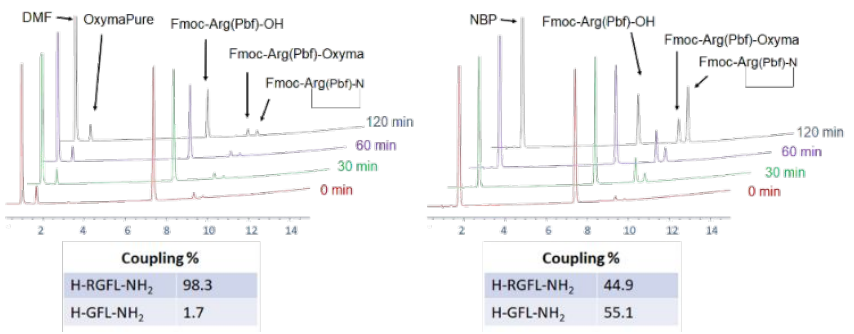

Figure 2. HPLC analysis of the reaction described in Scheme 2 using DMF and NBP as solvents at RT (See note at Figure 1 legend and Material and Methods section for HPLC conditions).

As pointed out in the previous study carried out with $\mathrm{NBP}^{17}$ the higher viscosity of NBP vs. DMF $\left(4.0 \mathrm{cP}\right.$ vs. $0.8 \mathrm{CP}$ at $\left.25^{\circ} \mathrm{C}\right)$ could explain the different performance of the coupling reaction, since the viscosity of the solvent may prevent the active species from reaching the functional groups inside the resin. Thus, with this idea in mind, we repeated the same experiment at $45^{\circ} \mathrm{C}$ in order to reduce the effect of viscosity during the coupling. Analysis of the supernatants revealed that both solvents rendered similar results in terms of ?lactam formation (Figure 3). However, analysis of the cleaved peptide showed an almost quantitative incorporation of the protected amino acid in the case of DMF and a substantial improvement in case of NBP, from $44.9 \%$ to $85.5 \%$ of coupling (Figure 3).
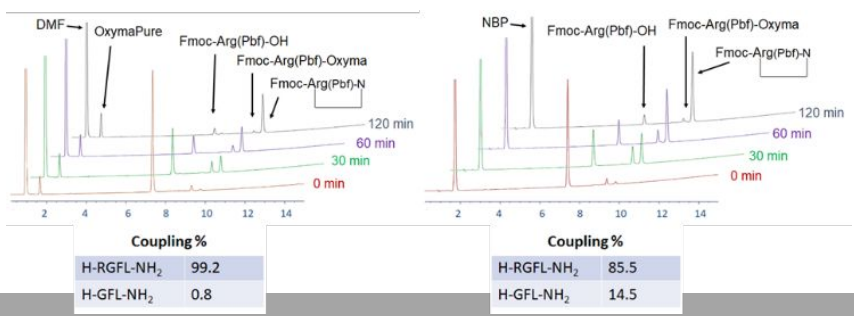
Figure 3. HPLC analysis of the reaction described in Scheme 2 using DMF and NBP as solvents at $45^{\circ} \mathrm{C}$. (See note at Figure 1 legend and Material and methods section for HPLC conditions)

Although these results represented a notable improvement, they were far from optimal. In order to fine-tune the coupling reaction in NBP to achieve quantitative incorporation of Fmoc-Arg(Pbf)-OH into the peptidyl-resin, the next experiments were performed only in NBP. Thus, in view of the favorable effect of heating in the previous experiment, the temperature was increased to $60^{\circ} \mathrm{C}$. Interestingly, analysis of the supernatant showed that no active species remained after $30 \mathrm{~min}$ of reaction and therefore no further coupling was possible after that time. Nevertheless, analysis of the cleaved peptide showed that the incorporation of the protected Arg reached 93\% (Figure 4A).

Although these results showed a satisfactory increase in the coupling reaction, the lack of active species in a short time meant that the reaction had reached a plateau, where no further coupling was possible. Thus, as this temperature $\left(60^{\circ} \mathrm{C}\right)$ on the one hand showed rapid consumption of reagent in both the coupling and side reaction, and on the other hand could be considered a limit for industrial purposes, the fine-tuning should be attempted using a strategy other than merely an increase in temperature.

As mentioned earlier, the higher viscosity of NBP may explain its poorer performance in coupling because of difficulty reaching the reactive points inside the resin. In this regard, it was envisaged that $\delta$-lactam formation was taking place and was accelerated during the time taken to reach the temperature after the addition of the solution mixture to the resin at RT. With this idea in mind, we attempted a new strategy. Using $25 \%$ of the total NBP needed for the coupling, the resin was swelled and allowed to equilibrate at $45^{\circ} \mathrm{C}$, followed by the addition of the mixture solution containing Fmoc$\operatorname{Arg}(\mathrm{Pbf})-\mathrm{OH}, \mathrm{OxymaPure}$, and $\mathrm{DIC}(1: 1: 1,1.5$ equiv.) in the remaining $75 \%$ volume of NBP $(0.075 \mathrm{M}$ final concentration). This procedure was the so-called pre-conditioning strategy.
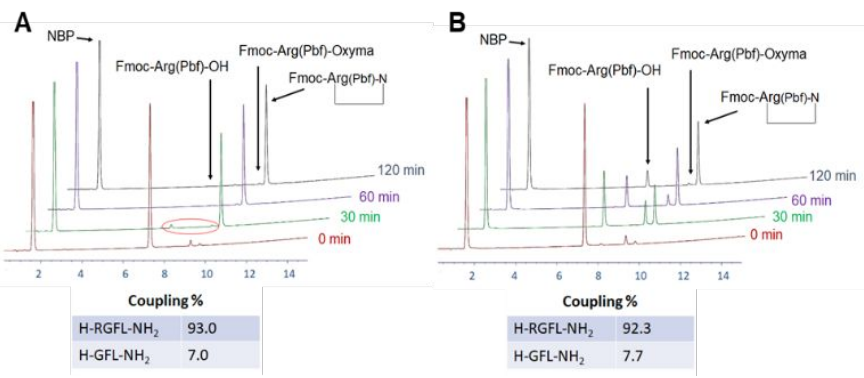

Figure $4 \mathrm{HPLC}$ analysis of the reaction described in Scheme 2 using NBP as solvent. A) at $60^{\circ} \mathrm{C}$. B) pre-conditioning at $45^{\circ} \mathrm{C}$ (See note at Figure 1 legend and Material and methods section for HPLC conditions).

Under this new strategy, analysis of the reaction supernatant showed that not all activated protected amino acid had been consumed after $2 \mathrm{~h}$, either because of coupling or conversion to $\delta$ lactam, as indicated by the presence of Fmoc-Arg(Pbf)-OH, which derived from the active species hydrolyzed in the reaction mixture or during the HPLC analysis (Figure 4B).

Analysis of the peptide after cleavage from the resin showed a coupling of $92.3 \%$. On the other hand, these pre-conditioning conditions at $45^{\circ} \mathrm{C}$ almost matched $(92.3 \%$ vs. $93.0 \%)$ the best coupling performance, which were achieved at $60^{\circ} \mathrm{C}$ and clearly better than the previous experiment at $45^{\circ} \mathrm{C}(85.5 \%$ vs $92.3 \%$, Figure 3 ). The observation that no full conversion to ?-lactam had been reached using the last conditions supported the idea that fine tuning them could render better results. In other words, the presence of some Fmoc-Arg(Pbf)-OH, presumably from Fmoc-Arg(Pbf)-Oxyma, indicated that the coupling was able to proceed further.
A

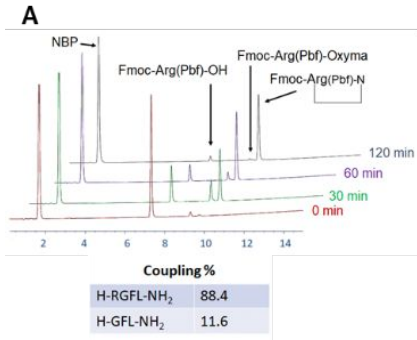

B

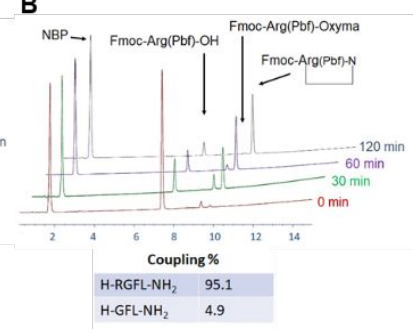

Figure 5. HPLC analysis of the reaction described in Scheme 2 using NBP as solvent by "in situ" activation. A) at concentration 0.075M. B) at concentration $0.15 \mathrm{M}$. (See note at Figure 1 legend and Material and methods section for HPLC conditions).

In view of the improved results achieved by pre-conditioning the temperature of the resin, the further experiments were carried out doing this pre-conditioning, adding a solution with the total amount (1:1, 1.5 equiv) of $\mathrm{Fmoc}-\mathrm{Arg}(\mathrm{Pbf})-\mathrm{OH}$ and OxymaPure to the resin to the resin. Then, once the mixture had reached $45^{\circ} \mathrm{C}$, the total amount of DIC (1.5 equiv.) was added. This procedure, which was called "in situ" activation, was run as described previously at reagents concentration of $0.075 \mathrm{M}$ but also using the same equivalents at a concentration of $0.15 \mathrm{M}$, a value very similar to that used in production mode SPPS. These assays highlighted the importance of concentration, since the one performed at $0.075 \mathrm{M}$ gave a coupling yield of $88.4 \%$ while that at $0.15 \mathrm{M}$ was $95.1 \%$. In both cases, but particularly in the second, a large amount of unreacted $\mathrm{Fmoc}-\mathrm{Arg}(\mathrm{Pbf})-\mathrm{OH}$ was found when the supernatants were analyzed (Figure 5). Therefore, to force the coupling of the remaining protected amino acid, the next experiment was performed by increasing the amount of coupling reagent, i.e. DIC, leading the ratio of reagents $\mathrm{Fmoc}-\mathrm{Arg}(\mathrm{Pbf})-\mathrm{OH} / \mathrm{DIC} / \mathrm{Oxyma}$ to 1:1.2:1 (this means 1.8 equiv of DIC with respect to the growing peptide)following the same procedure as before. This increment in the DIC ratio yielded a satisfactory improvement of the result, raising coupling to $98.2 \%$ (Figure $6 \mathrm{~A}$ ). This result could be because using this in situ activation, the formation of the Oxyma ester take place when the peptidyl-resin is already solvated in NBP at $45^{\circ} \mathrm{C}$, and therefore the peptide bond formation does not compete so unfavorably with ? -lactam formation. 
Encouraged by this result, the next experiment was designed under the same concentration, temperature and equivalent as before but modifying the way in which DIC was added. The total amount DIC was divided into two equal portions, the first was added when the peptidyl-resin and Fmoc-Arg(Pbf)-OH/OxymaPure mixture in the NBP solution reached $45^{\circ} \mathrm{C}$ and the second after $30 \mathrm{~min}$ of reaction. In this case, the coupling achieved was slightly better (98.7\%) (Figure 6B). Although slight, this increase was considered positive because it

$$
\text { A }
$$

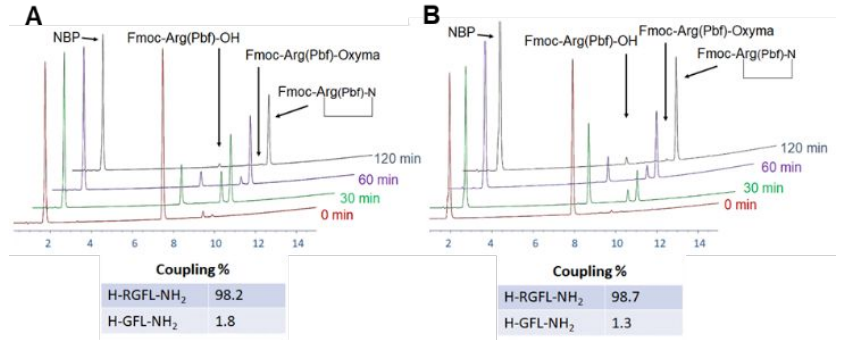

showed that this strategy does not impair the process and thus finetuning can be attempted.

Figure 6. HPLC analysis of the reaction described in Scheme 2 using NBP as solvent by "in situ" activation using Fmoc-Arg(Pbf)OH/DIC/Oxyma 1:1.2:1 ratio. A) DIC addition in one portion. B) DIC addition in two portions. (See note at Figure 1 legend and Material and methods section for HPLC conditions).

The rationale behind this experiment was to protect $\mathrm{Fmoc}-\mathrm{Arg}(\mathrm{Pbf})$ $\mathrm{OH}$ from conversion into ?-lactam. As shown in Scheme 3, when half the DIC (0.90 equiv.) is added, a part of Fmoc- $\mathrm{Arg}(\mathrm{Pbf})-\mathrm{OH}$ is converted to its $\mathrm{O}$-acyl isourea derivative [Fmoc- $\mathrm{Arg}(\mathrm{Pbf})$-DliU], ( $\max$ 0.90 equiv.), which immediately reacts with the rest of the Fmoc$\mathrm{Arg}(\mathrm{Pbf})-\mathrm{OH}$ ( 0.60 equiv.) to give the symmetrical anhydride [[Fmoc$\left.\operatorname{Arg}(\mathrm{Pbf})]_{2} \mathrm{O}\right]$ and/or with OxymaPure to give the Oxyma active ester [Fmoc-Arg(Pbf)-Oxyma]. Then, the [Fmoc-Arg(Pbf) $]_{2} \mathrm{O}$ can react either with the amino peptidyl-resin or with Oxyma pure. In either case, one molecule of $\mathrm{Fmoc}-\mathrm{Arg}(\mathrm{Pbf})-\mathrm{OH}$ ( 0.60 equiv.) will be released and this will be activated when the second portion of DIC is added (Blue pathway in Scheme 3).

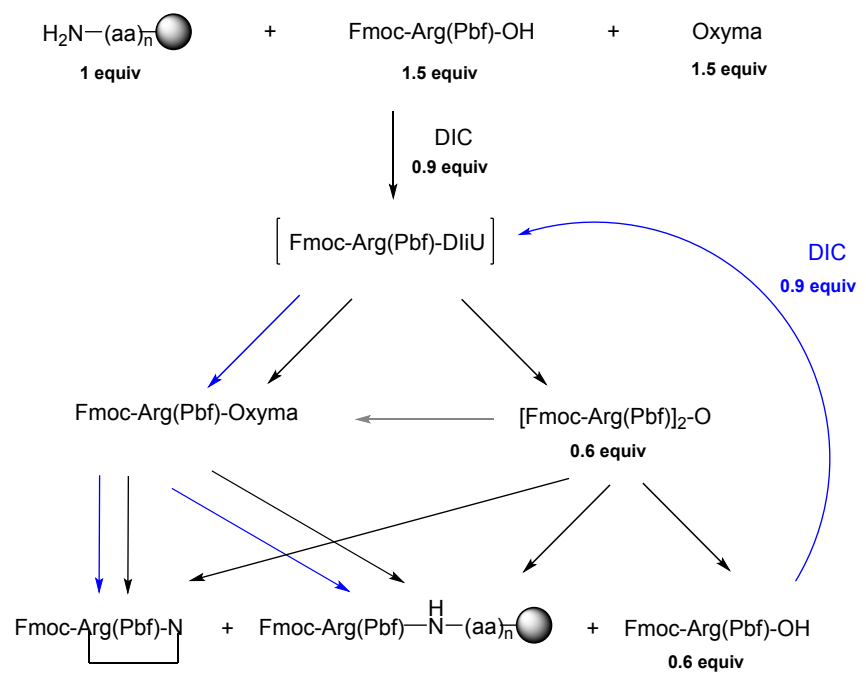

Scheme 3. Synthetic pathway involved in the coupling of Fmoc$\operatorname{Arg}(\mathrm{Pbf})-\mathrm{OH}$.
Finally, the same strategy was attempted but adding some fresh Fmoc-Arg(Pbf)-OH together with the second portion of DIC. Only

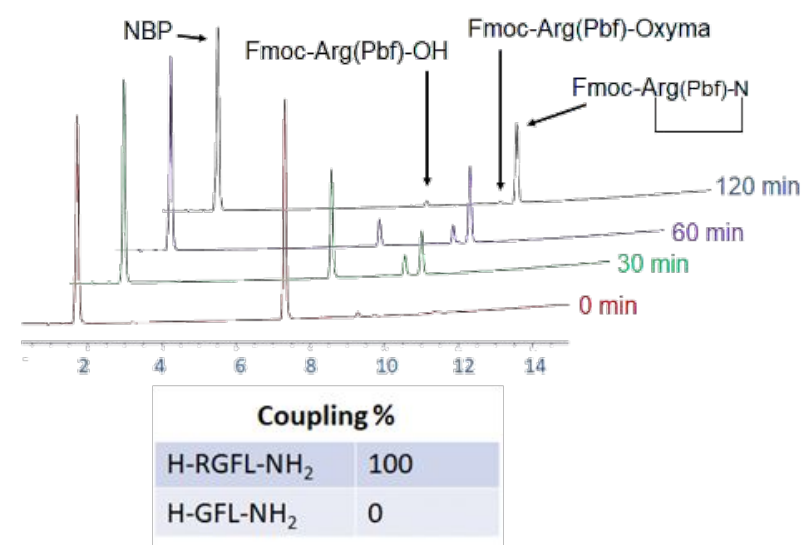

0.25 equiv. of the protected amino acid with respect to the growing peptide was added, resulting in a total usage of 1.75 equiv. of the protected Arg. In this case, the coupling reaction reached $100 \%$.

Figure 7. HPLC analysis of the reaction described in Scheme 2 using NBP as solvent by "in situ" activation using Fmoc-Arg(Pbf)$\mathrm{OH} / \mathrm{DIC} / O x y m a$ 1:1.2:1 ratio. DIC addition in two portions, 0.25 equiv of protected Arg in the second DIC addition. (See note at Figure 1 legend and Material and methods section for HPLC conditions).

To validate this procedure, first the assay was performed studying the coupling over a more hindered amino acid, since Gly lacks of side chain. The choice was Val because it is a $\beta$-branched amino acid, thus the coupling reaction is more difficult. The tripeptide H-Val-Phe-Leu$\mathrm{NH}$-Rink-amide-resin was prepared and Fmoc-Arg(Pbf)-OH was coupled following the last protocol described above. Incorporation exceeded $99 \%$. Next, the strategy was applied to the synthesis of the lineal precursor of the cyclic RGD peptide. The sequence H-DfKRG$\mathrm{OH}$ was synthesized on chlorotrityl (CTC)-resin and the peptide was obtained with excellent purity without the presence of the des-Arg

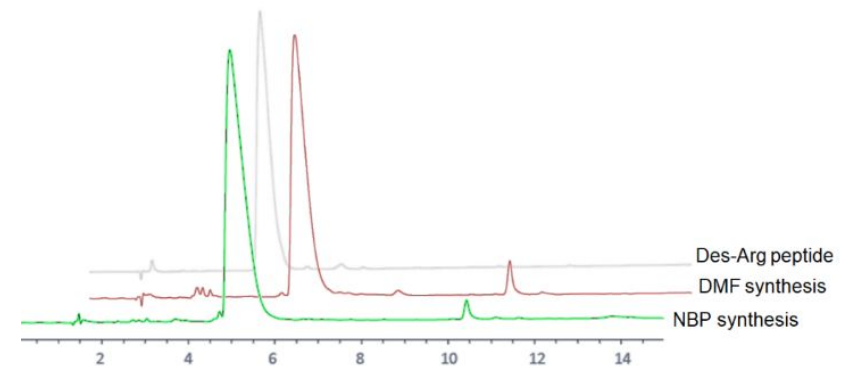

peptide. The quality of the crude was comparable to that obtained by standard protocols using DMF as solvent (Figure 8 ).

Figure 8. HPLC Analysis of the lineal precursor of the cyclic RGD peptide. Green: total synthesis in NBP, Arg coupled under methodology developed. Red: standard synthesis in DMF. Grey: DesArg peptide synthesized as standard.

\section{Experimental}

Materials 
All reagents and solvents were purchased from commercial suppliers and used without further purification. Fmoc amino acids, Fmoc-Rink Amide AM resin (loading $0.74 \mathrm{mmol} / \mathrm{g}$ ) and 2-CTC resin were purchased from Iris Biotech. NBP (TamiSolveNxG) was purchased from Eastman, OxymaPure was a gift from Luxembourg Biotech., and $\mathrm{N}, \mathrm{N}$-Diisopropylethylamine (DIEA) and 4-methylpiperidine were supplied by Sigma-Aldrich. Organic solvents Dimethylformamide (DMF), 2-Methyltetrahydrofuran (2-MeTHF), cyclopenthylmethyl eter (CPME) and HPLC quality acetonitrile $\left(\mathrm{CH}_{3} \mathrm{CN}\right)$ were purchased from Merck. Milli-Q water was used for RP-HPLC analyses. Analytical HPLC was performed on an Agilent 1100 system using a Phenomenex Aeris ${ }^{\mathrm{TM}} \mathrm{C} 18(3.6 \mu \mathrm{m}, 4.6 \times 150 \mathrm{~mm})$ column, with flow rate of 1.0 $\mathrm{mL} / \mathrm{min}$ and UV detection at $220 \mathrm{~nm}$. Chemstation software was used for data processing. Buffer A: $0.1 \%$ TFA in $\mathrm{H}_{2} \mathrm{O}$; buffer $\mathrm{B}: 0.1 \%$ TFA in $\mathrm{CH}_{3} \mathrm{CN}$. LC-MS was performed on a Shimadzu 2020 UFLC-MS using an YMC-Triart C18 $(5 \mu \mathrm{m}, 4.6 \times 150 \mathrm{~mm})$ column. Buffer A: 0.1\% formic acid in $\mathrm{H}_{2} \mathrm{O}$; buffer $\mathrm{B}$ : $0.1 \%$ formic acid in $\mathrm{CH}_{3} \mathrm{CN}$.

\section{$\delta$-Lactam formation study}

1. $0.1 \mathrm{M}$ solution of Fmoc-Arg(Pbf)-OH/DIC/OxymaPure (1:1:1) was prepared in NBP or DMF, $10-\mu \mathrm{L}$ sample was taking at $0,30,60$, and
120 min. mixture and diluted to $1 \mathrm{~mL}$ with $\mathrm{CH}_{3} \mathrm{CN}$. From this solution $1 \mu \mathrm{L}$ was injected and analyzed by analytical RP-HPLC. General Method: $30-95 \%$ B into A in 15 min.

2. From the different coupling conditions (Table 1) $10-\mu \mathrm{L}$ sample of the supernatant was taking at $0,30,60$, and 120 min. mixture and diluted to $1 \mathrm{~mL}$ with $\mathrm{CH}_{3} \mathrm{CN}$. From this solution $1 \mu \mathrm{L}$ was injected and analyzed by analytical RP-HPLC. General Method: $30-95 \%$ B into A in $15 \mathrm{~min}$.

\section{SPPS}

All peptides were assembled manually in plastic syringes fitted with a polyethylene porous disc, according to $\mathrm{Fmoc} / \mathrm{tBu}$ methodology by mean of DIC/ Oxyma as coupling agents. Fmoc removal was carried out by treatment with a solution of $20 \%$ of 4 -methylpiperidine. The solvent was NBP and DMF for comparison.

The tripeptidylresin (H-GFL-resin) was prepared on Rink-amide-PS (loading $0.74 \mathrm{mmol} / \mathrm{g}$ ), coupling of each residue was performed using a ratio Fmoc-AA-OH/DIC/Oxyma 1:1:1, 1.5 equiv. in NBP or DMF at $45^{\circ} \mathrm{C}$ for $1 \mathrm{~h}$. The different conditions for coupling of Fmoc-Arg (Pbf)$\mathrm{OH}$ are detailed in Table 1. Peptides were cleaved from the resin, by treating with TFA/TIS/H2O (95:2.5:2.5) for $1 \mathrm{~h}$ at $\mathrm{rt}$ and precipitated with chilled CPME. HPLC analysis method for coupling quantification was $10-25 \%$ B into $A$.

Table 1: Coupling conditions for Fmoc-Arg(Pbf)-OH

\begin{tabular}{|c|c|c|c|c|}
\hline Entry & $\begin{array}{c}\text { Fmoc-Arg(Pbf)-OH/DIC/ Oxyma } \\
\text { Ratio }\end{array}$ & Eq. & $\begin{array}{c}{[\mathrm{Fmoc}-\operatorname{Arg}(\mathrm{Pbf})-\mathrm{OH}]} \\
\text { (M) }\end{array}$ & $\mathrm{T}\left({ }^{\circ} \mathrm{C}\right)$ \\
\hline 1 & 1:1:1 & 1.5 & 0.075 & RT \\
\hline 2 & 1:1:1 & 1.5 & 0.075 & $45^{\circ} \mathrm{C}$ \\
\hline 3 & 1:1:1 & 1.5 & 0.075 & $60^{\circ} \mathrm{C}$ \\
\hline 4 & $1: 1: 1^{1}$ & 1.5 & 0.075 & $45^{\circ} \mathrm{C}$ \\
\hline 5 & $1: 1: 1^{2}$ & 1.5 & 0.075 & $45^{\circ} \mathrm{C}$ \\
\hline 6 & $1: 1: 1^{2}$ & 1.5 & 0.15 & $45^{\circ} \mathrm{C}$ \\
\hline 7 & $1: 1.2: 1^{2}$ & 1.5:1.8:1.5 & 0.15 & $45^{\circ} \mathrm{C}$ \\
\hline 8 & $1: 1.2: 1^{3}$ & 1.5:1.8:1.5 & 0.15 & $45^{\circ} \mathrm{C}$ \\
\hline 9 & $1: 1.2: 1^{4}$ & $1.75: 1.8: 1.5$ & 0.15 & $45^{\circ} \mathrm{C}$ \\
\hline \multicolumn{5}{|c|}{$\begin{array}{l}\text { Pre-conditioned resin in Fmoc- } \mathrm{Arg}(\mathrm{Pbf})-\mathrm{OH} / \mathrm{Oxyma} \text { in solvent at } 45^{\circ} \mathrm{C} \text {, followed by DIC addition. } \\
\text { Pre-conditioned resin in Fmoc- } \mathrm{Arg}(\mathrm{Pbf})-\mathrm{OH} / \mathrm{Oxyma} \text { in solvent at } 45^{\circ} \mathrm{C} \text {, followed by addition: } \\
\quad \mathrm{t}=00.6 \text { equiv of DIC } \\
\mathrm{t}=30 \text { min } 0.6 \text { equiv of } \mathrm{DIC}\end{array}$} \\
\hline \multicolumn{5}{|c|}{$\begin{array}{l}{ }^{4} \text { Pre-conditioned resin in Fmoc- } \mathrm{Arg}(\mathrm{Pbf})-\mathrm{OH} / \mathrm{Oxymas} \text { in solvent at } 45^{\circ} \mathrm{C} \text {, followed by addition: } \\
\qquad \mathrm{t}=0 \text { 0 } 0.6 \text { equiv of } \mathrm{DIC} \\
\mathrm{t}=30 \text { min } 0.25 \text { eq. } \mathrm{Fmoc}-\mathrm{Arg}(\mathrm{Pbf})-\mathrm{OH}+0.6 \text { equiv of } \mathrm{DIC}\end{array}$} \\
\hline
\end{tabular}

RGD and desArgRGD peptides were synthesized on 2-CTC resin $(0.05$ $\mathrm{mmol}$ scale, loading $0.56 \mathrm{mmol} / \mathrm{g}$ ). The incorporation of the first amino acid was done with Fmoc-Gly-OH (1.5 equiv) in 2-Me-THF in presence of base DIEA (10 equiv) for 2 hours, then capping with 
$\mathrm{MeOH}(50 \mu \mathrm{L})$ for $30 \mathrm{~min}$ at room temperature. The rest of amino acids were coupled as described above and Arg was incorporated following the conditions Entry 9 described in Table 1. Peptides were cleaved from the resin, by treating with TFA/TIS/H2O (95:2.5:2.5) for $1 \mathrm{~h}$ at $\mathrm{rt}$ and precipitated with chilled CPME. HPLC analysis method was $5-20 \%$ B into $A$. The peptides were characterized by LCMS.

\section{Conclusions}

The potential suitability of NBP as a green solvent candidate to substitute the DMF in SPPS was reported in a previous study. In the present work, we focused on the coupling of Fmoc-Arg(Pbf)-OH, a highly problematic amino acid because of its ability to react intramolecularly, leading to its $\delta$-lactam derivative. The kinetic comparison of $\delta$-lactamization in DMF and NBP in the presence of a stoichiometric ratio of DIC/oxymapure proved only slightly superior in DMF than NBP at RT but was much faster in the green solvent when the temperature was raised to $60^{\circ} \mathrm{C}$. When the assay was performed in the presence of the nucleophile, the peptidyl-resin, to study the competition between $\delta$-lactam formation and incorporation of the protected amino acid to the resin, the results revealed the key role of solvent viscosity for the coupling reaction. While at RT, the incorporation of the protected Arg was almost quantitative in DMF and less than $50 \%$ in NBP, at $45^{\circ} \mathrm{C}$ the incorporation using NBP exceeded $85 \%$.

After systematically modifying the different parameters that could influence the coupling reaction, such as temperature, concentration or mode of addition of the reagents, a successful protocol was established. Thus, the optimal temperature was fixed at $45^{\circ} \mathrm{C}$, the concentration of the reagents at $0.15 \mathrm{M}$ and the Fmoc-Arg(Pbf)$\mathrm{OH} / \mathrm{DIC} / \mathrm{oxymapure}$ ratio at 1:1.2:1. The procedure involved preconditioning of the NBP solution containing the protected Arg, oxymapure and the peptidyl-resin at the fixed temperature, then addition of half the DIC and after $30 \mathrm{~min}$ the other half, together with 0.25 equiv. of $\mathrm{Fmoc}-\operatorname{Arg}(\mathrm{Pbf})-\mathrm{OH}$.

Thus, using NBP as a solvent, it was possible to safely introduce Fmoc-Arg(Pbf)-OH in SPPS under conditions compatible with industrial requirements, namely moderate temperature and keeping excesses to a minimum: 1.75 equiv. For the protected amino acids, 1.8 equiv. of DIC, and 1.5 equiv. Of oxymapure. It is envisaged that this procedure will be further applied for the incorporation of other protected amino acids in a SPPS approach.

Finally, this work has evidenced that the study of the supernatant of a SPPS reaction can bring key information to fine-tune the overall process.

\section{Conflicts of interest}

There are no conflicts to declare

\section{Acknowledgements}

The work was funded in part by the following: the National Research Foundation (NRF) (\# 105892 and Blue Sky's Research Programme \# 110960) and the University of KwaZulu-Natal (South Africa); and the Spanish Ministry of Science, Innovation, and Universities (CTQ2015-67870-P) and the Generalitat de Catalunya (2017 SGR 1439) (Spain).

\section{Notes and references}

1. A. Henninot, J. C. Collins and J. M. Nuss, J. Med. Chem., 2017, 61, 1382-1414.

2. J. L. Lau and M. K. Dunn, Bioorg. Med. Chem., 2018, 26, 27002707.

3. B. G de la Torre and F. Albericio, Molecules, 2019, 24, 809.

4. D. Al Shaer, O. Al Musaimi, F. Albericio and B. G. de la Torre, Pharmaceuticals, 2019, 12, 52.

5. O. Al Musaimi, D. Al Shaer, B. de la Torre and F. Albericio, Pharmaceuticals, 2018, 11, 42.

6. R. B. Merrifield, J. Am. Chem. Soc., 1963, 85, 2149-2154.

7. G. R. Marshall, Pept. Sci., 2008, 90, 190-199.

8. T. Bruckdorfer, O. Marder and F. Albericio, Curr. Pharm. Biotechnol., 2004, 5, 29-43.

9. A. A. Zompra, A. S. Galanis, O. Werbitzky and F. Albericio, Future Med. Chem., 2009, 1, 361-377.

10. B. L. Bray, Nat. Rev. Drug Discov., 2003, 2, 587.

11. L. A. Carpino, H. Shroff, S. A. Triolo, E.-S. M. Mansour, H. Wenschuh and F. Albericio, Tetrahedron Lett., 1993, 34, 78297832.

12. A. Isidro-Llobet, M. N. Kenworthy, S. Mukherjee, M. E. Kopach, K. Wegner, F. Gallou, A. G. Smith and F. Roschangar, J. Org. Chem., 2019, 84, 4615-4628.

13. Y. E. Jad, G. A. Acosta, S. N. Khattab, B. G. de la Torre, T. Govender, H. G. Kruger, A. El-Faham and F. Albericio, Amino Acids, 2016, 48, 419-426.

14. A. Kumar, Y. E. Jad, J. M. Collins, F. Albericio, B. G. de la Torre, ACS Sustain. Chem. Eng., 2018, 6, 8034-8039.

15. O. Al Musaimi, Y. E. Jad, A. Kumar, A. El-Faham, J. M. Collins, A. Basso, B. G. de la Torre and F. Albericio, Org. Process Res. Dev, 2018, 22, 1809-1816.

16. A. Kumar, A. Thompson-Adewumi, K. Nandhini, J. M. Collins, F. Albericio and B. G. de la Torre, Org. Process Res. Dev, 2019, 23 1096-1100.

17. J. Lopez, S. Pletscher, A. Aemissegger, C. Bucher and F. Gallou, Org. Process Res. Dev, 2018, 22, 494-503.

18. T.-L. Hwang, K. Ranganathan, Y.-Q. Fang, R. D. Crockett, S. Osgood and S. Cui, Org. Process Res. Dev, 2018, 22, 1007-1014.

19. C. Allolio, A. Magarkar, P. Jurkiewicz, K. Baxová, M. Javanainen, P. E. Mason, R. Šachl, M. Cebecauer, M. Hof and D. Horinek, Proc. Natl. Acad. Sci., 2018, 115, 11923-11928.

20. R. Subirós-Funosas, R. Prohens, R. Barbas, A. El-Faham and F. Albericio, Chem. Eur. J., 2009, 15, 9394-9403. 


\section{Supporting Information}

\section{Successful Development of a Method for the Incorporation of Fmoc- $\operatorname{Arg}(\mathbf{P b f})-\mathrm{OH}$ in Solid-Phase Peptide Synthesis using $N$-Butylpyrrolidinone (NBP) as a Green Solvent}

Beatriz G. de la Torre, *a Ashish Kumar, ${ }^{\mathrm{a}, \mathrm{b}}$ Mahama Alhassan, ${ }^{\mathrm{b}}$ Christoph Bucher, ${ }^{\mathrm{c}}$ Fernando Albericio, ${ }^{* b, d, e}$ John Lopez *c,

${ }^{a}$ KwaZulu-Natal Research Innovation and Sequencing Platform (KRISP), School of Laboratory Medicine and Medical Sciences, College of Health Sciences, University of KwaZulu-Natal, Durban 4041, South Africa

beptide Science Laboratory, School of Chemistry and Physics, University of KwaZulu-Natal, Durban 4001, South Africa

'Novartis Pharma AG, Novartis Campu Campus, WSJ-145-5.51, CH-4056 Basel, Switzerland;

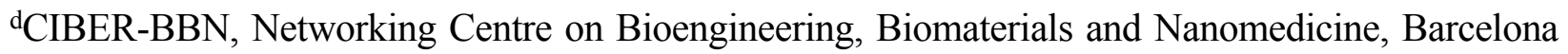
Science Park, University of Barcelona, 08028 Barcelona, Spain

eDepartment of Organic Chemistry, University of Barcelona, 08028 Barcelona, Spain 


\section{Table of content \\ 1 General Information \\ 2 HPLC: Supernatant analysis of $\boldsymbol{\delta}$-lactamization study [Fmoc- \\ : $\quad \mathrm{S} 1-\mathrm{S} 15$ \\ $\operatorname{Arg}(\mathrm{Pbf})-\mathrm{OH}$ : DIC : OxymaPure] in different conditions after 0, 30, 60, $120 \mathrm{~min}$ \\ 3 HPLC-Coupling quantification of Tripeptide (H-GFL-NH $)_{2}$ vs \\ : $\quad$ S16-S24 \\ Tetrapeptide (H-RGFL-NH2) after $120 \mathrm{~min}$ in different conditions \\ 4 HPLC: SPPS of Linear precursor of RGD Peptide (H-DfKRG-OH) : $\quad$ S25-S27}

\section{General Information}

All reagents and solvents were purchased from commercial suppliers and used without further purification. Fmoc amino acids, Fmoc-Rink Amide AM resin (loading $0.74 \mathrm{mmol} / \mathrm{g}$ ) and 2-CTC resin were purchased from Iris Biotech. NBP (TamiSolveNxG) was purchased from Eastman, OxymaPure was a gift from Luxembourg Biotech., and N,N-Diisopropylethylamine (DIEA) and 4-methylpiperidine were supplied by Sigma-Aldrich. Organic solvents Dimethylformamide (DMF), 2-Methyltetrahydrofuran (2-MeTHF), cyclopenthylmethyl eter (CPME) and HPLC quality acetonitrile $\left(\mathrm{CH}_{3} \mathrm{CN}\right)$ were purchased from Merck. Milli-Q water was used for RP-HPLC analyses. Analytical HPLC was performed on an Agilent 1100 system using a Phenomenex Aeris ${ }^{\mathrm{TM}} \mathrm{C} 18(3.6 \mu \mathrm{m}, 4.6 \times 150 \mathrm{~mm})$ column, with flow rate of $1.0 \mathrm{~mL} / \mathrm{min}$ and $\mathrm{UV}$ detection at $220 \mathrm{~nm}$

\section{HPLC Method:}

Supernatant analysis of $\delta$-lactamization study: $30-95 \%$ B into A in 15 min.

Coupling quantification: $10-25 \% \mathrm{~B}$ into $\mathrm{A}$

Linear precursor of RGD Peptide: 5-20\% B into A 
HPLC-S1. Fmoc-Arg(Pbf)-OH : DIC : OxymaPure [1:1:1], at RT, in DMF, in absence of nucleophile.

$0 \min$

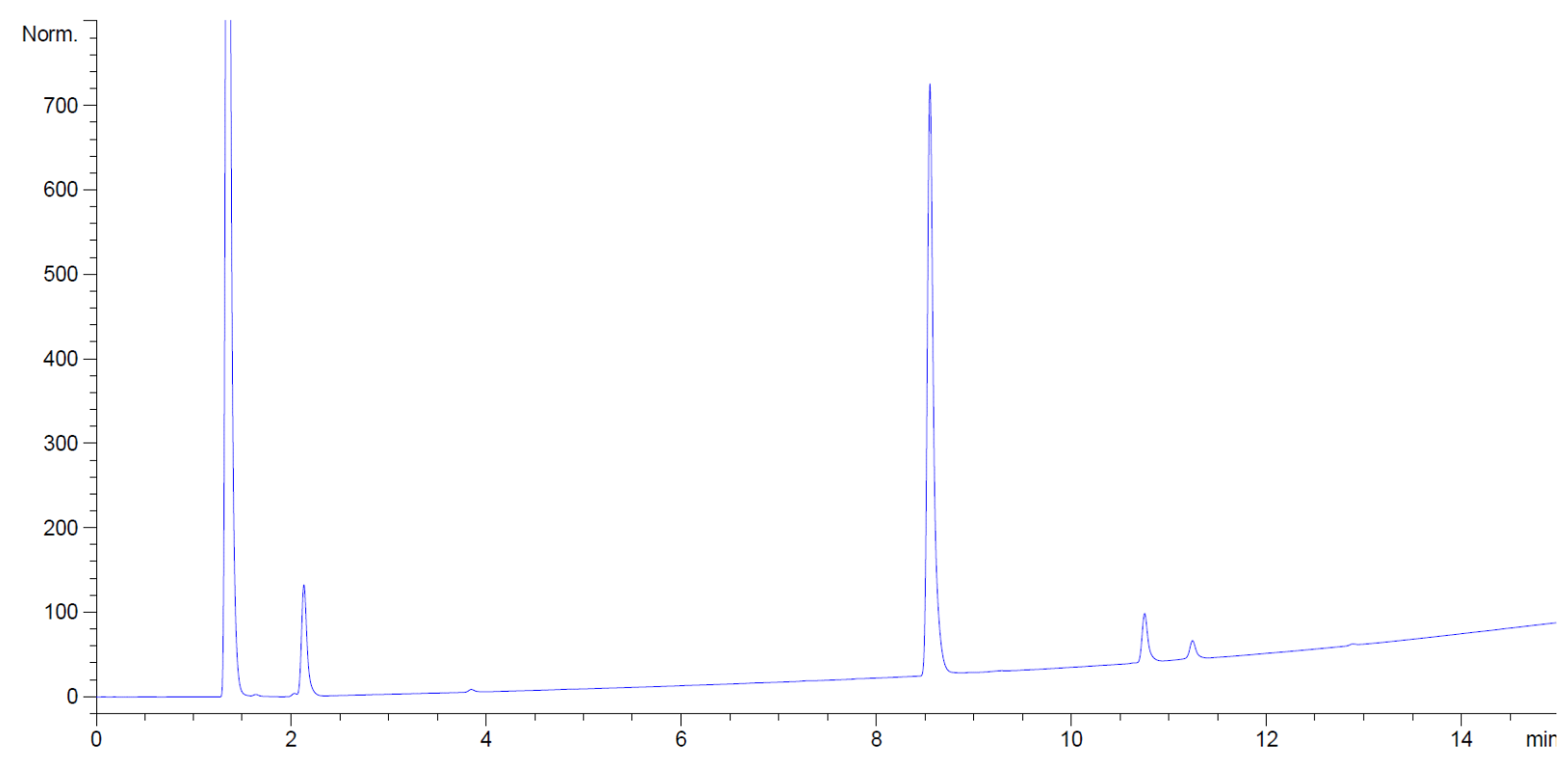

$30 \min$

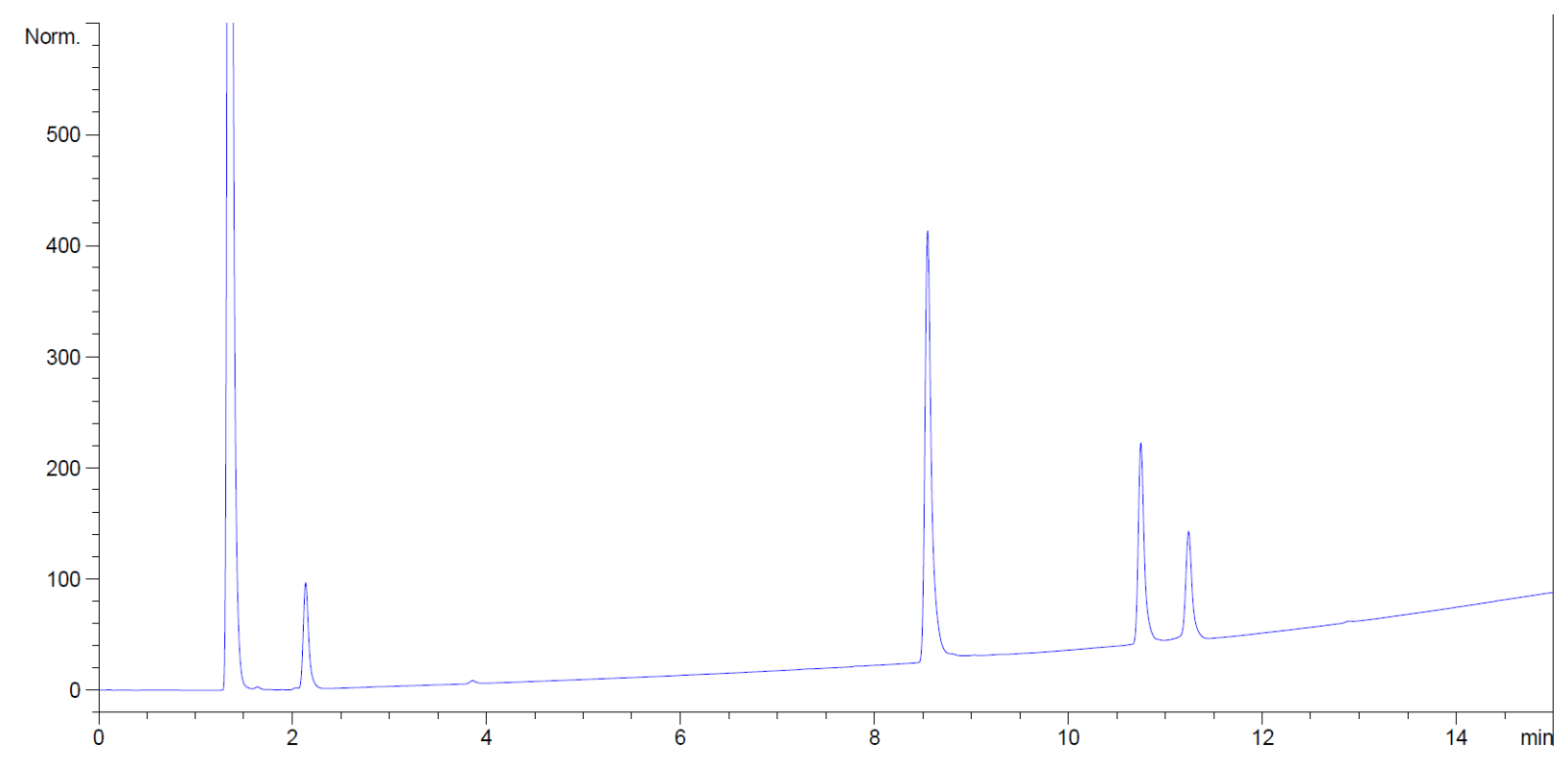


$60 \mathrm{~min}$

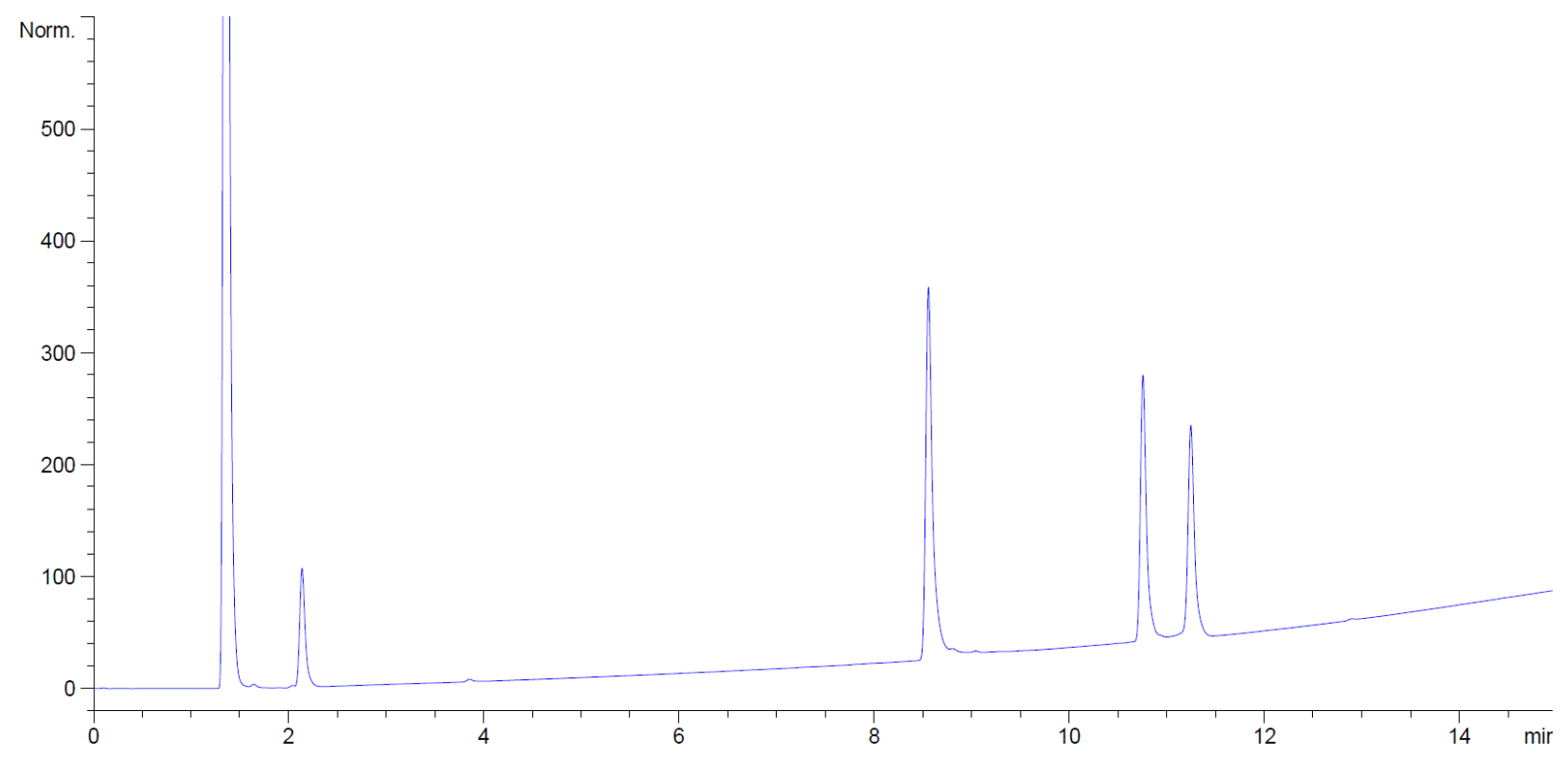

\section{$120 \mathrm{~min}$}

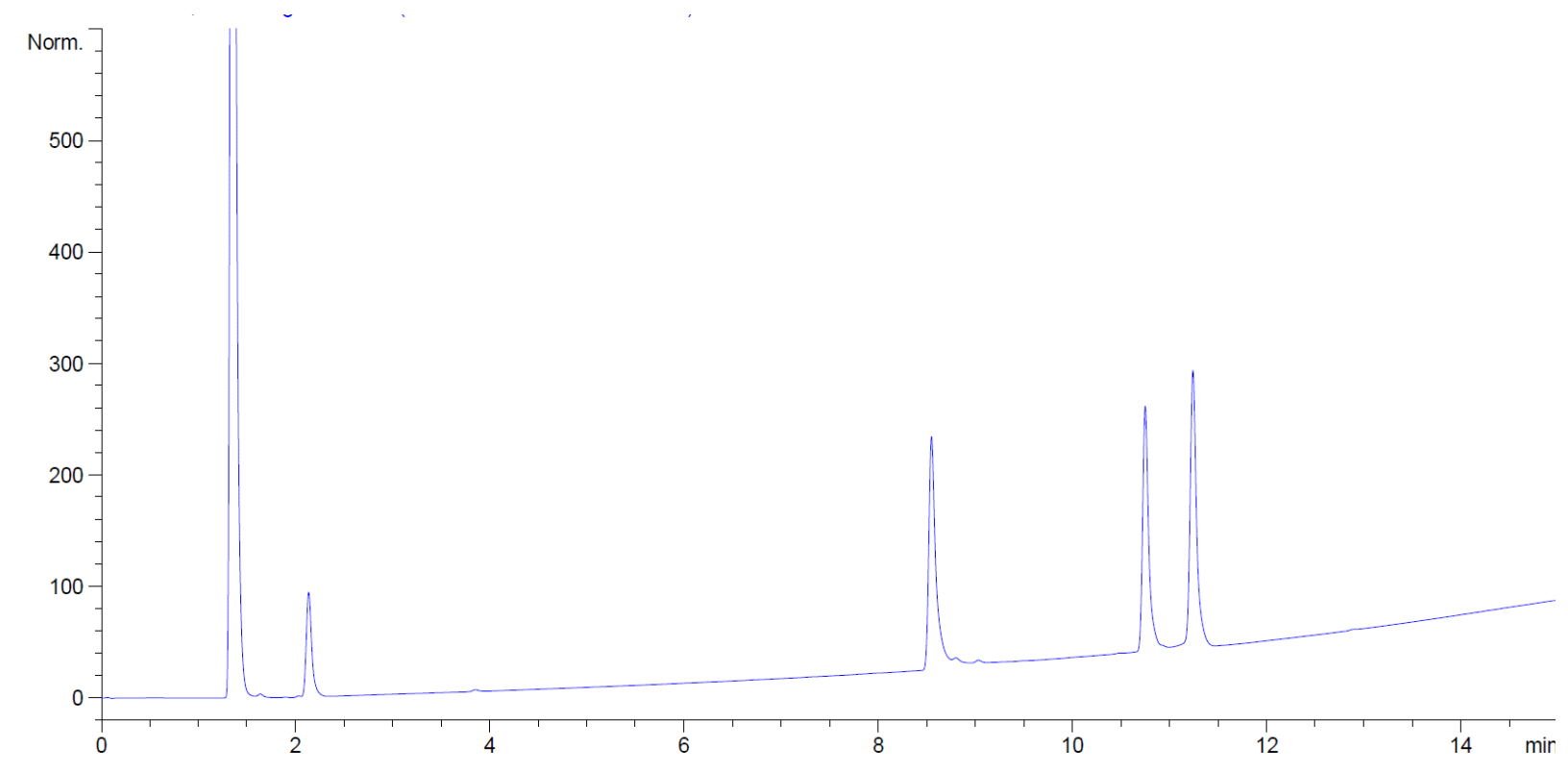


HPLC-S2. Fmoc-Arg(Pbf)-OH : DIC : OxymaPure [1:1:1], at RT, in NBP, in absence of nucleophile.

$0 \min$

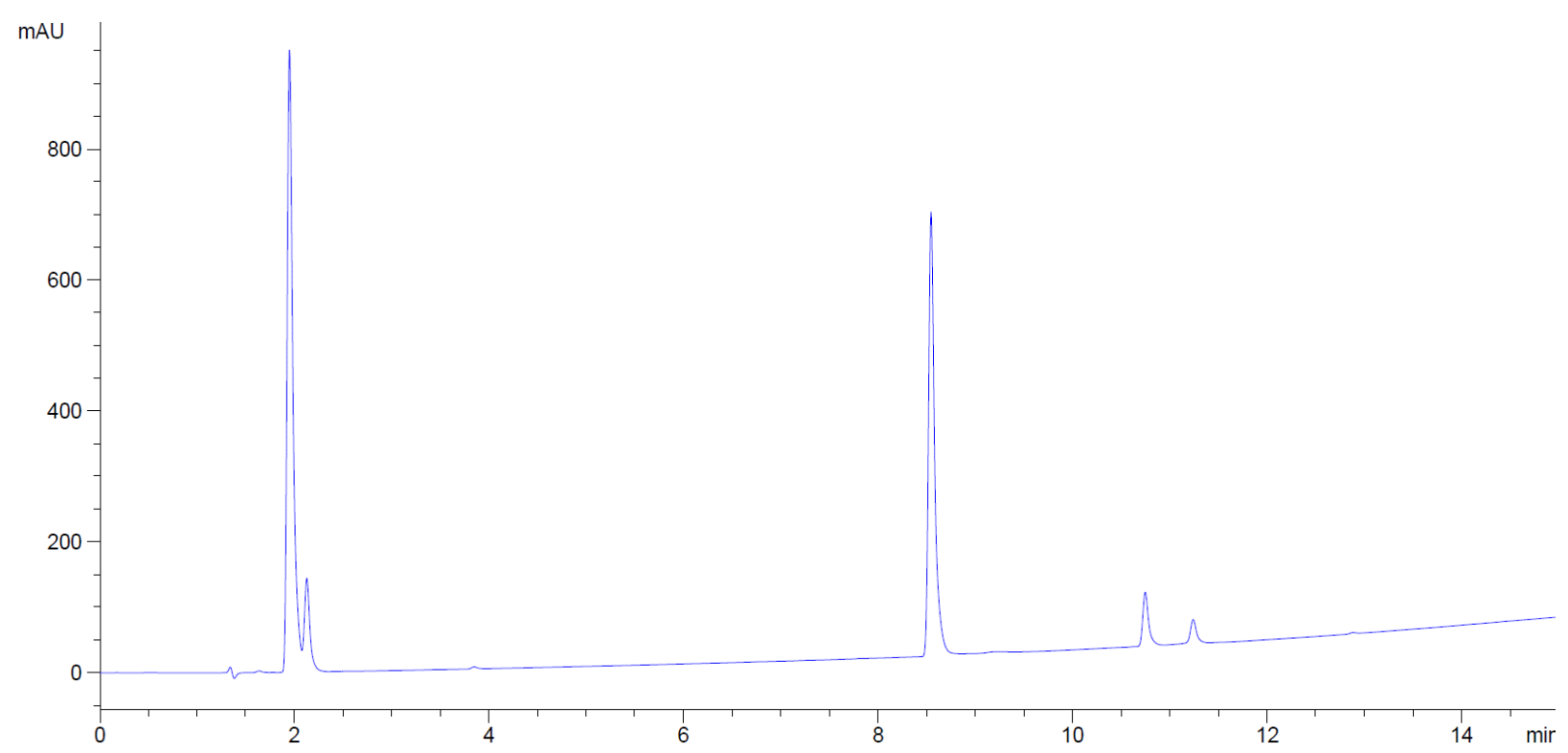

$30 \min$

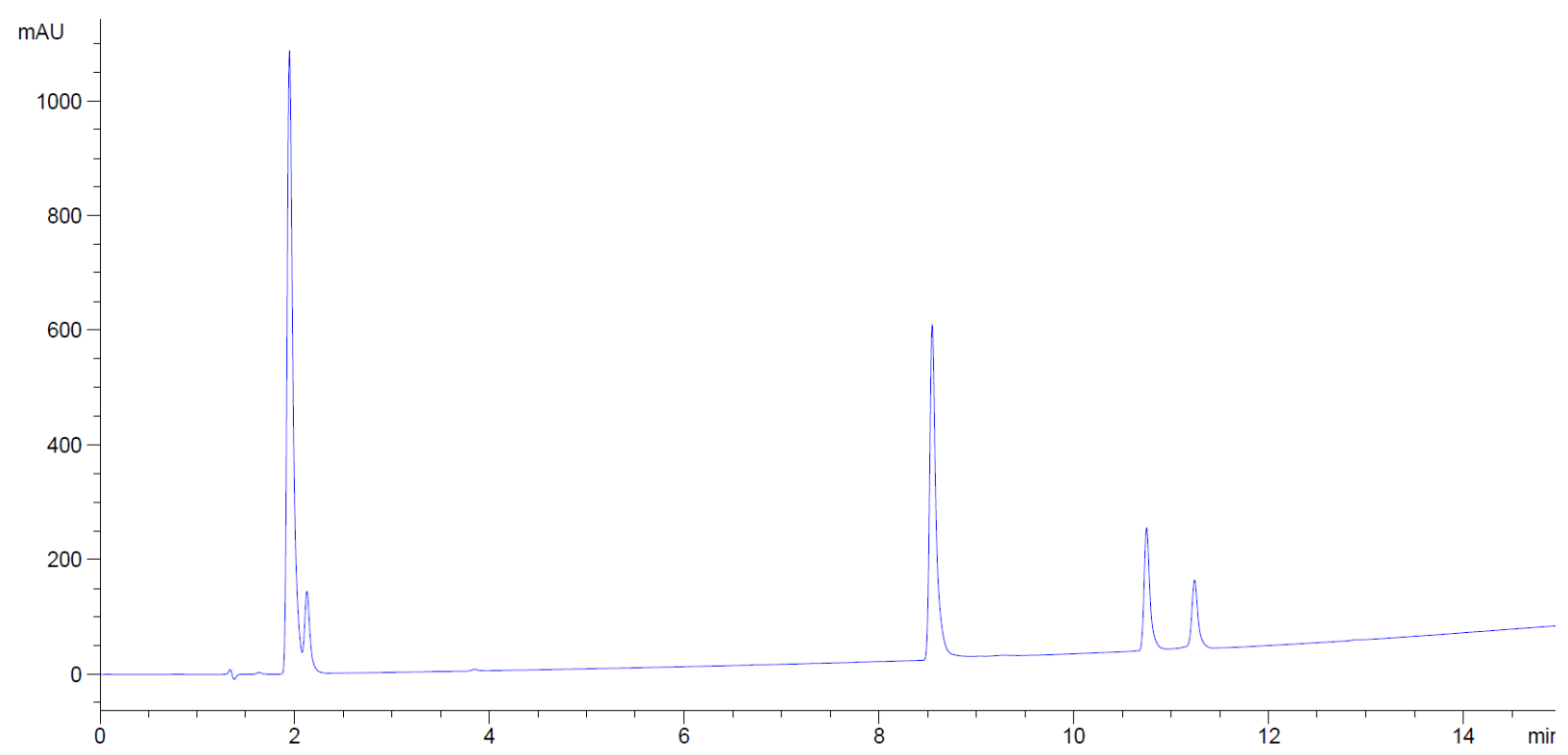

$60 \min$ 


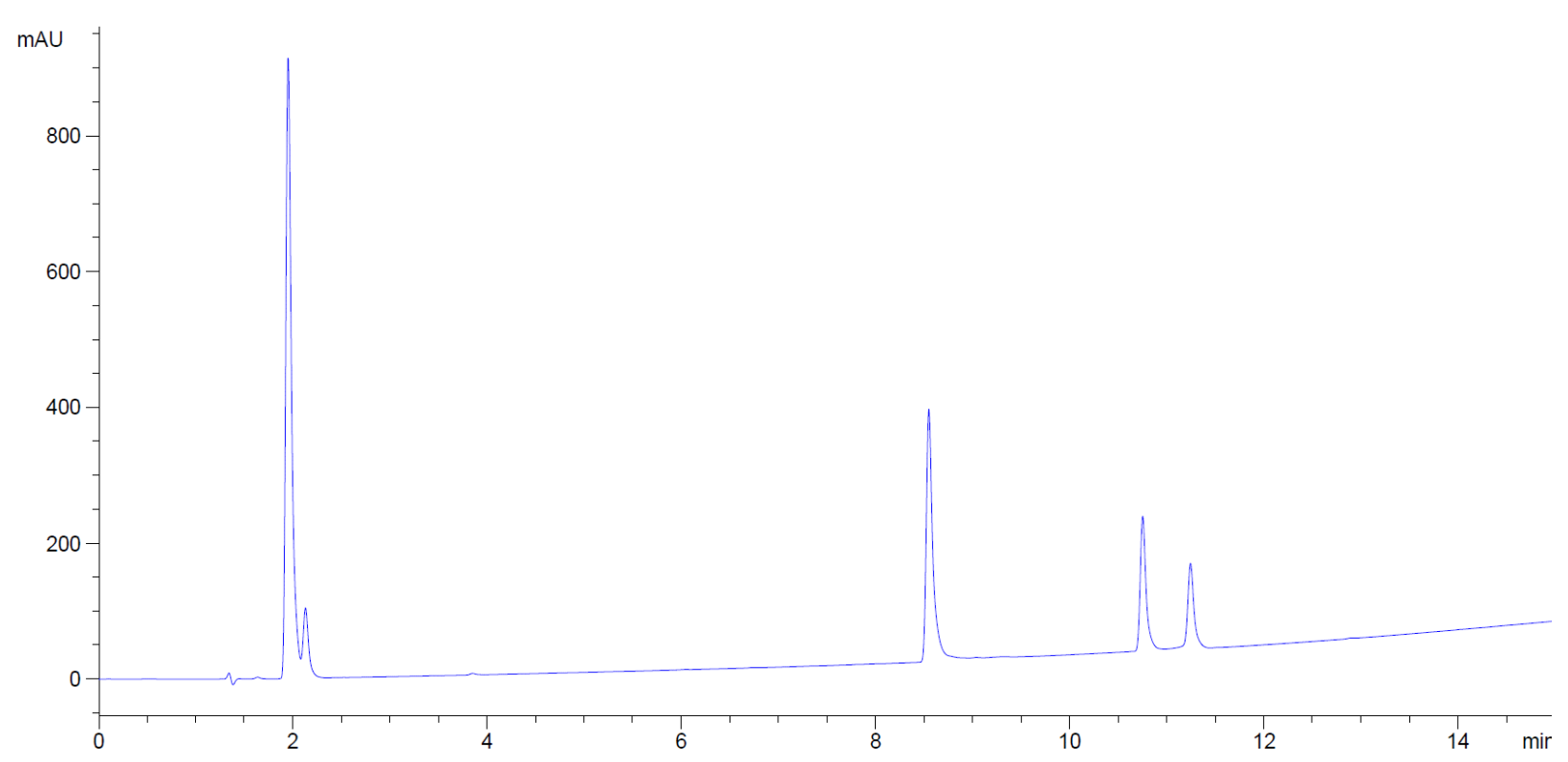

$120 \mathrm{~min}$

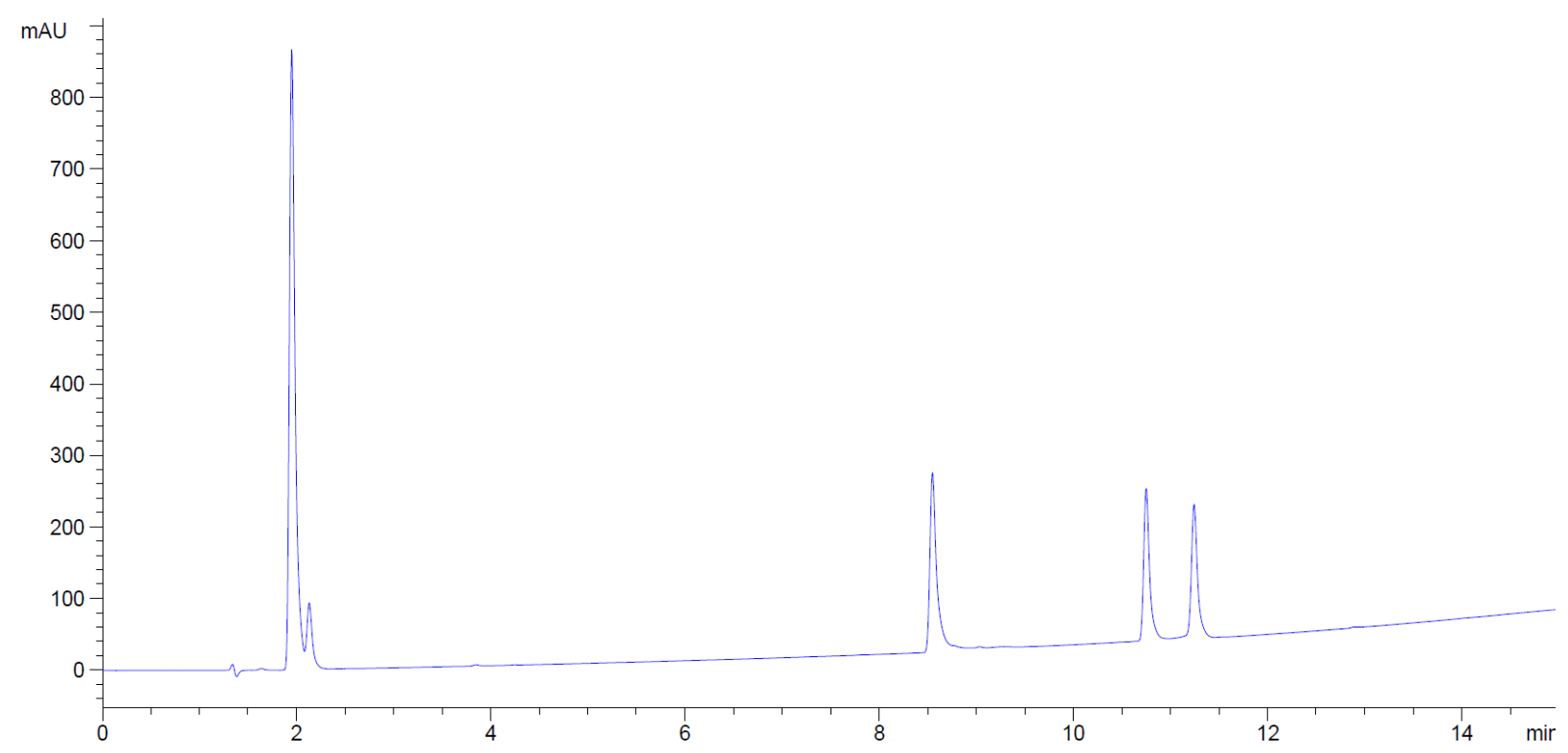

HPLC-S3. Fmoc-Arg(Pbf)-OH : DIC : OxymaPure [1:1:1], at $60^{\circ} \mathrm{C}$, in DMF, in absence of nucleophile. 
0 min

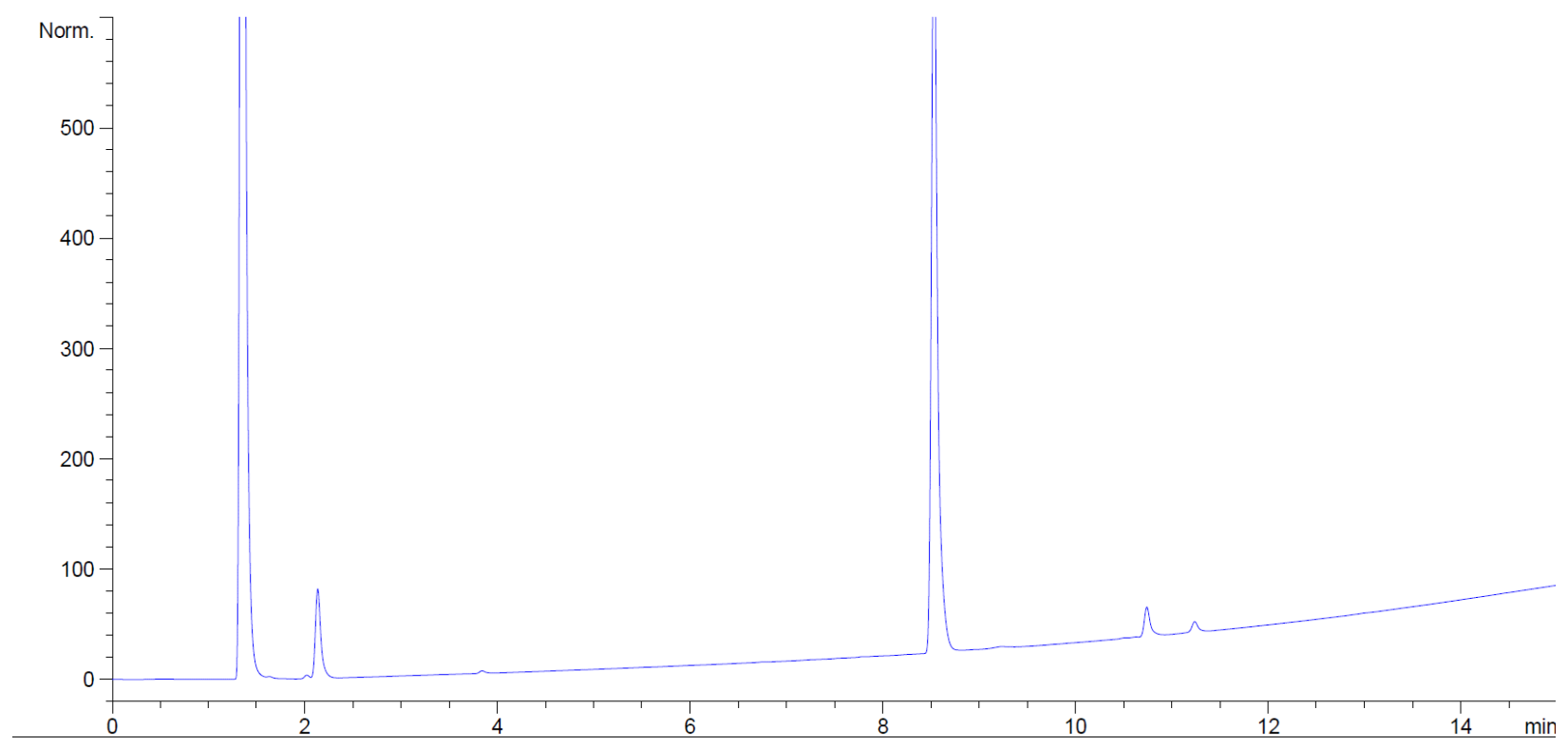

$30 \mathrm{~min}$

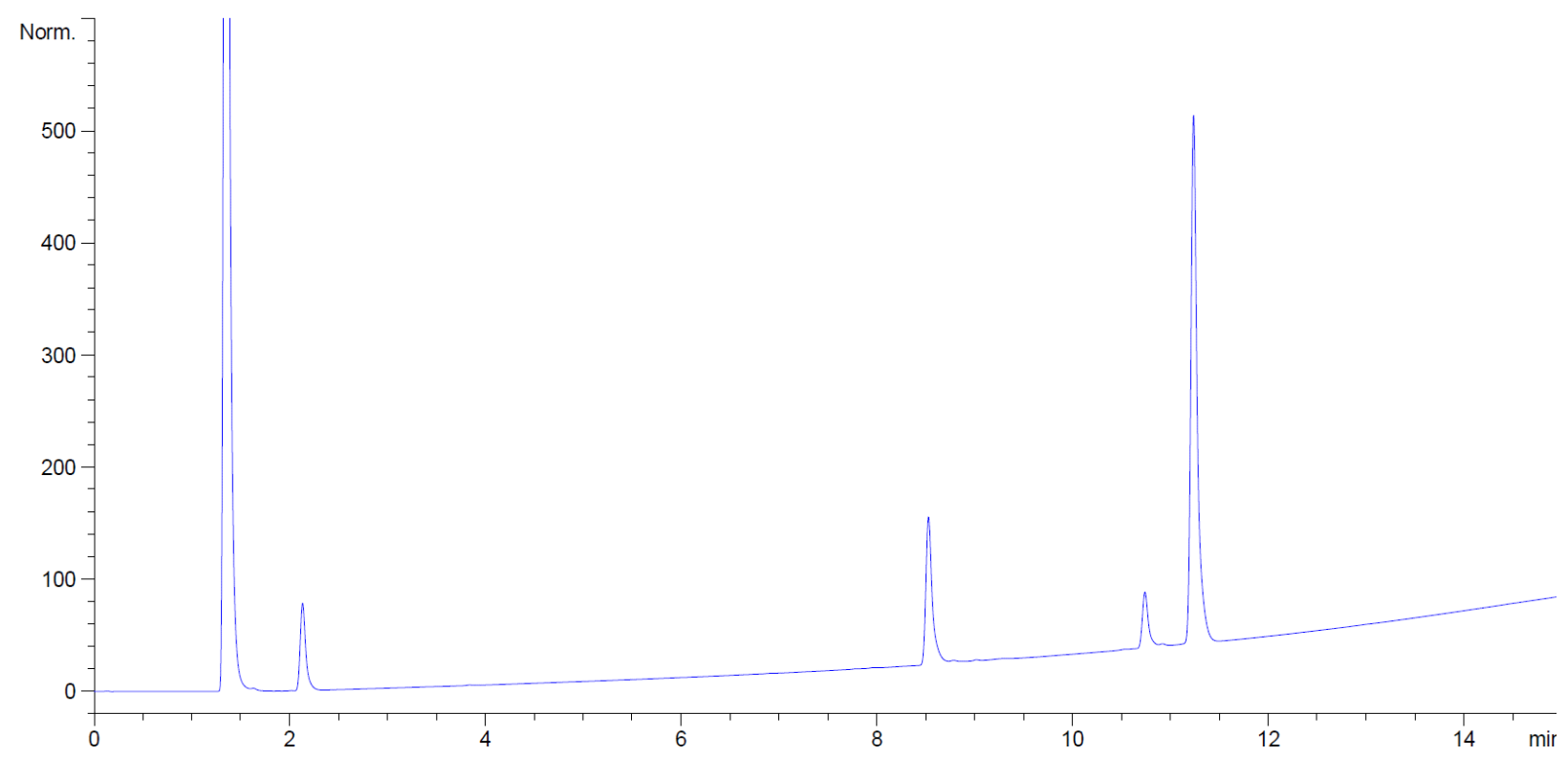

$60 \mathrm{~min}$ 


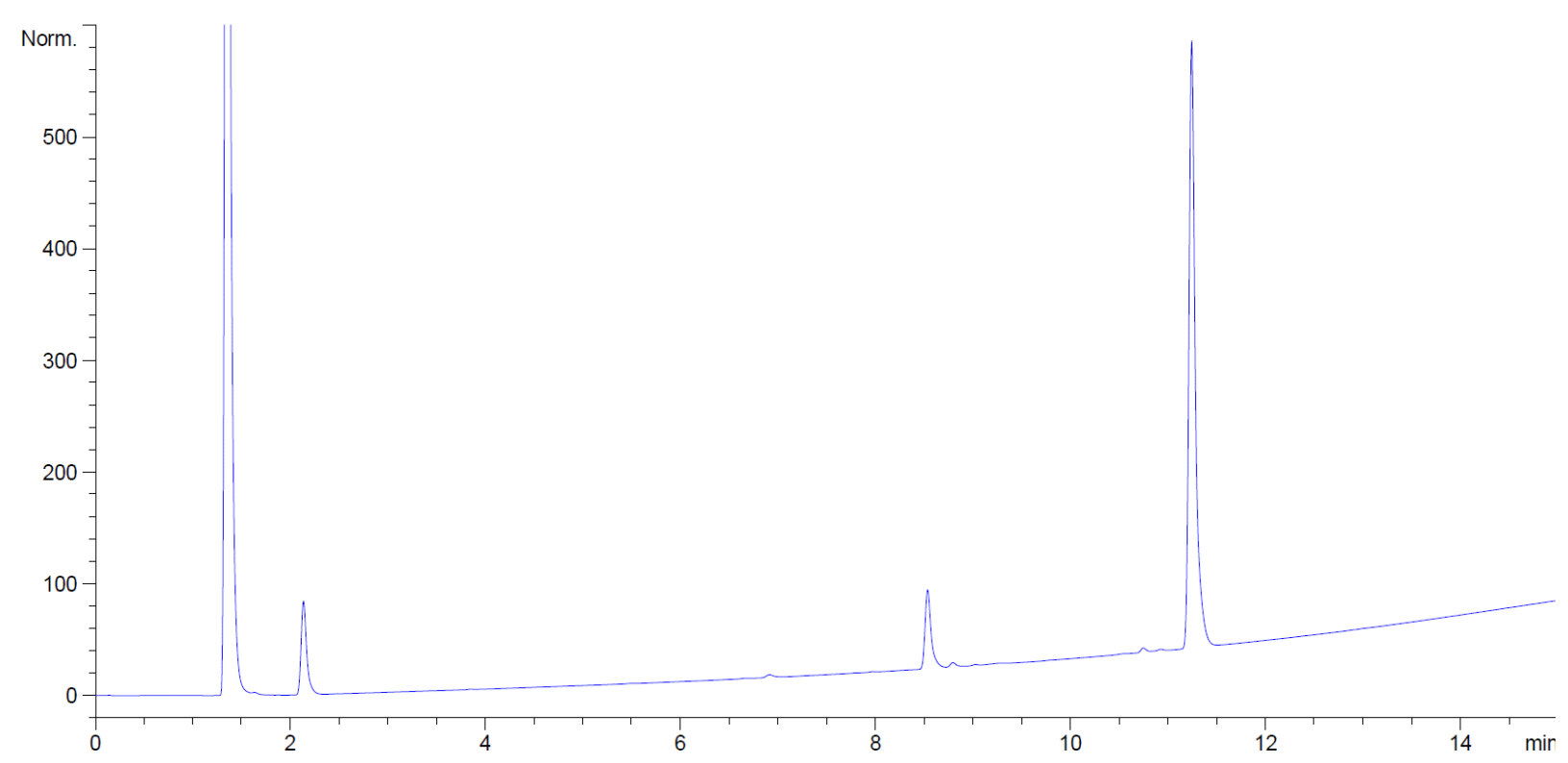

$120 \mathrm{~min}$

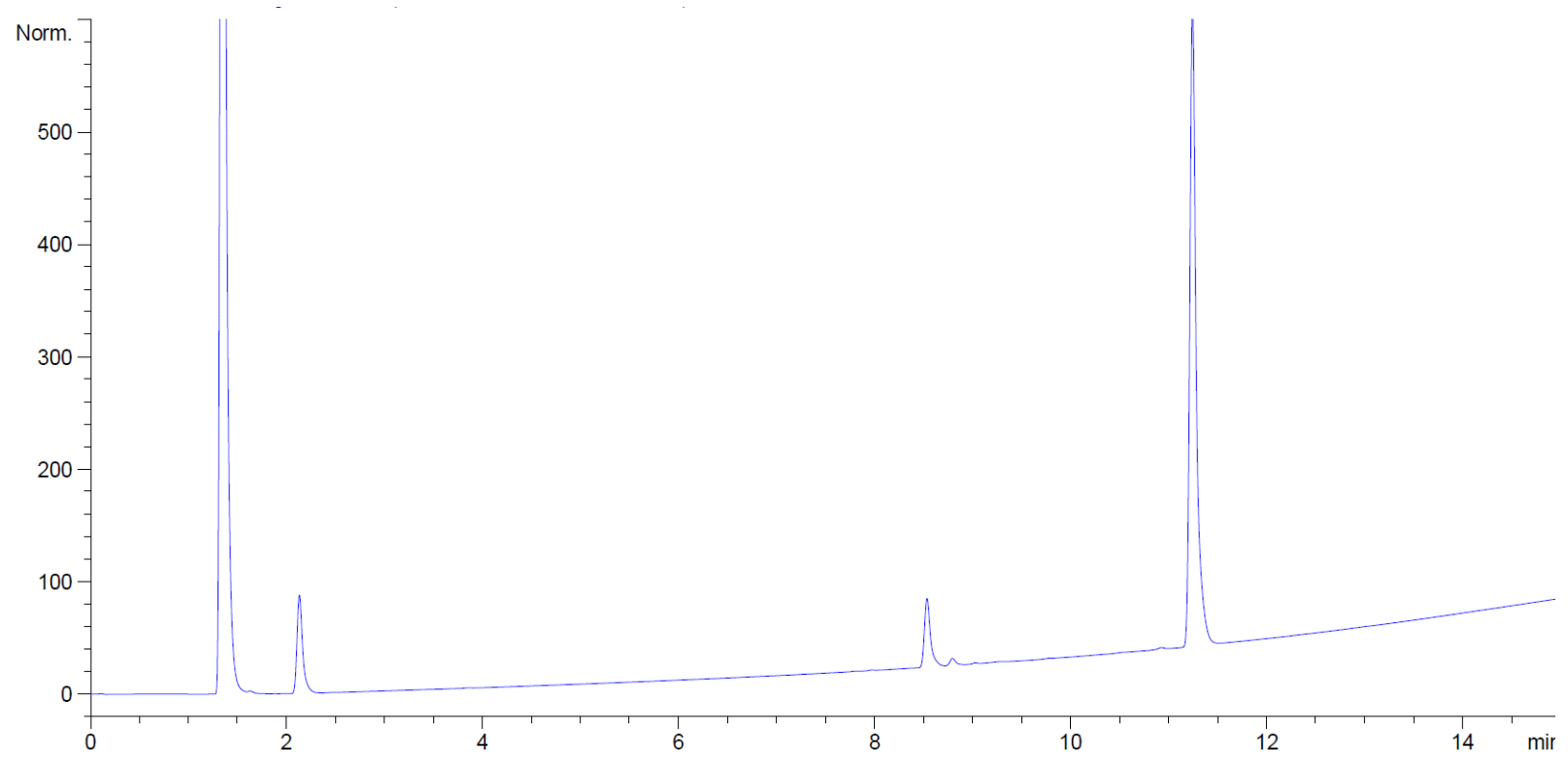

HPLC-S4. Fmoc-Arg(Pbf)-OH : DIC : OxymaPure [1:1:1], at $60^{\circ} \mathrm{C}$, in NBP, in absence of nucleophile. 


\section{0 min}

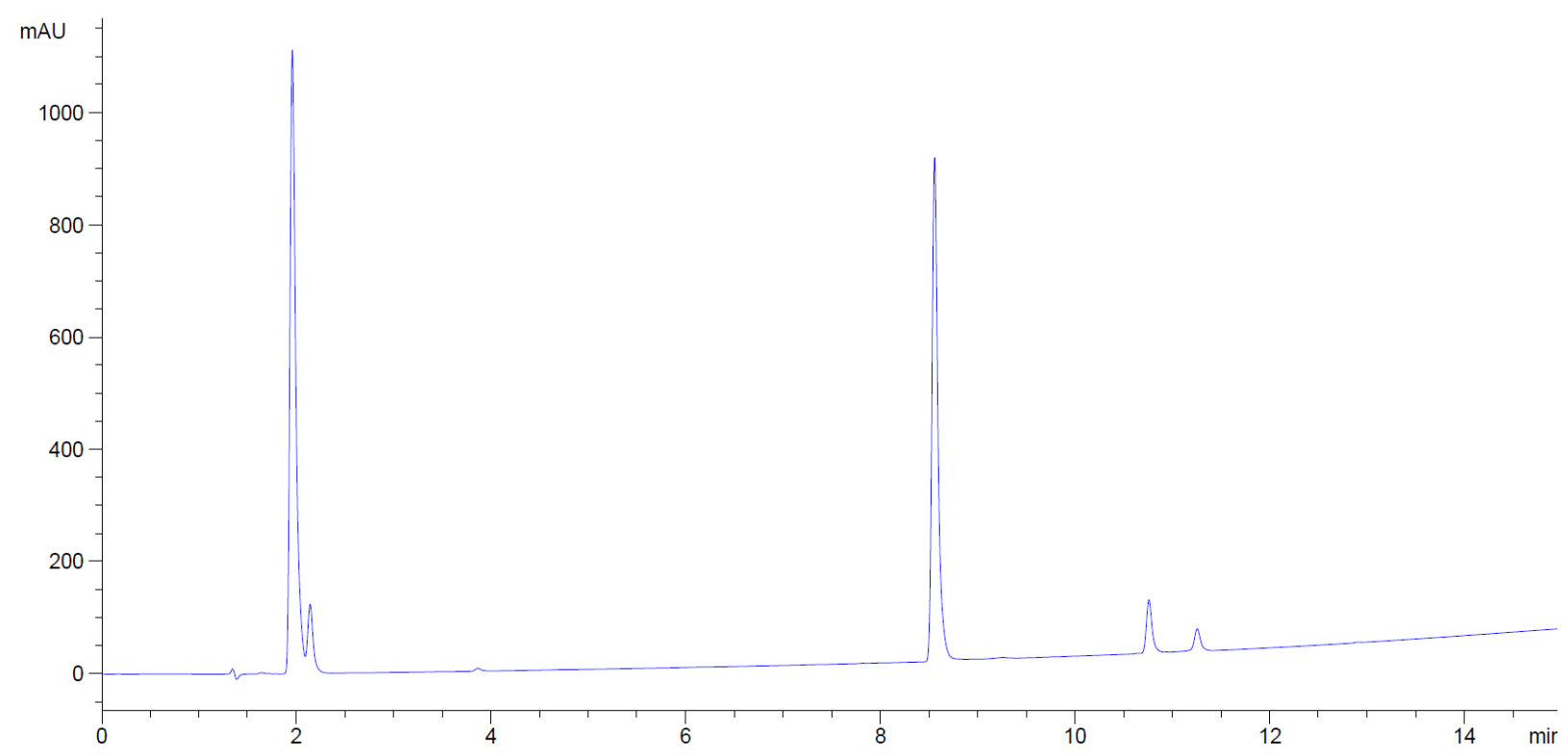

$30 \mathrm{~min}$

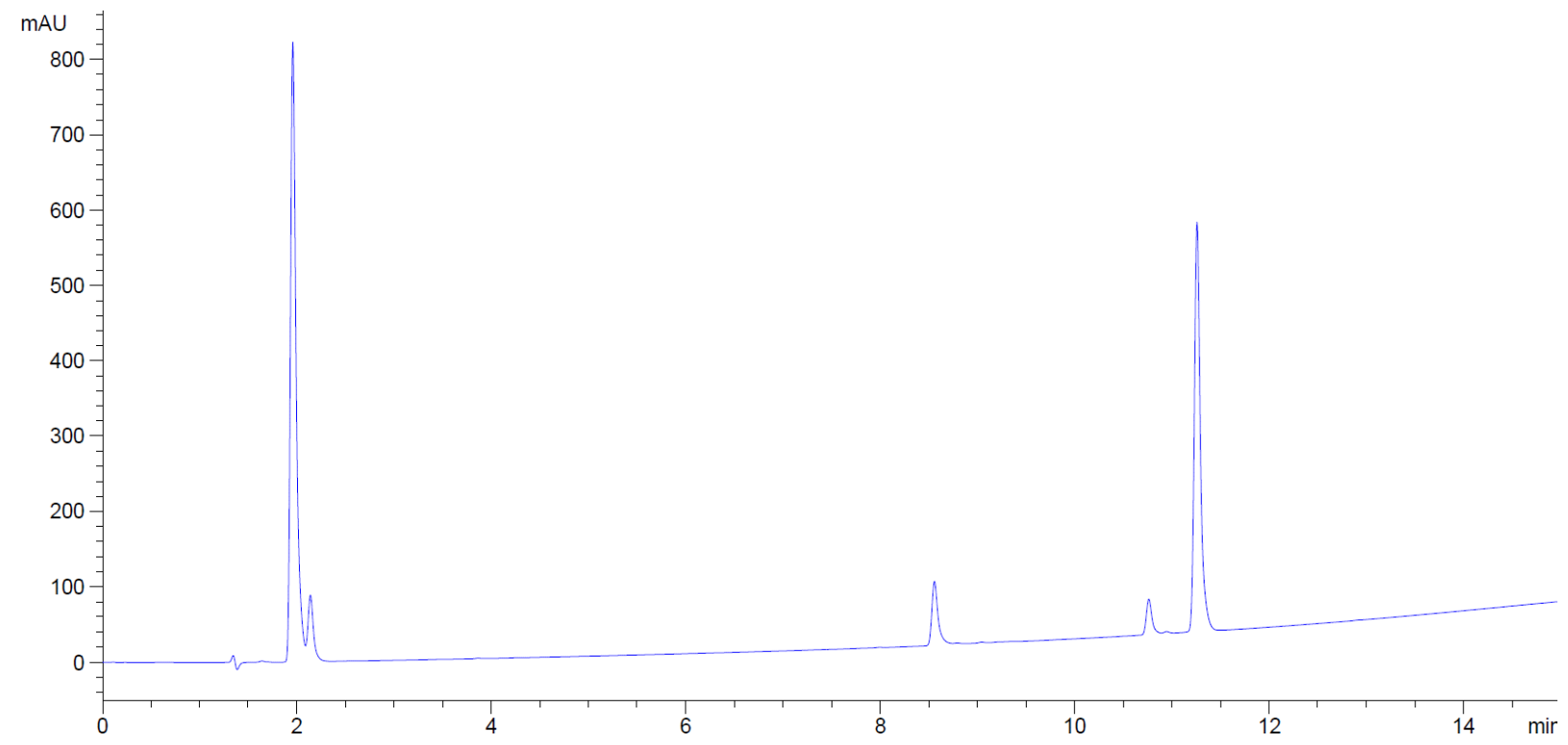

$60 \mathrm{~min}$ 


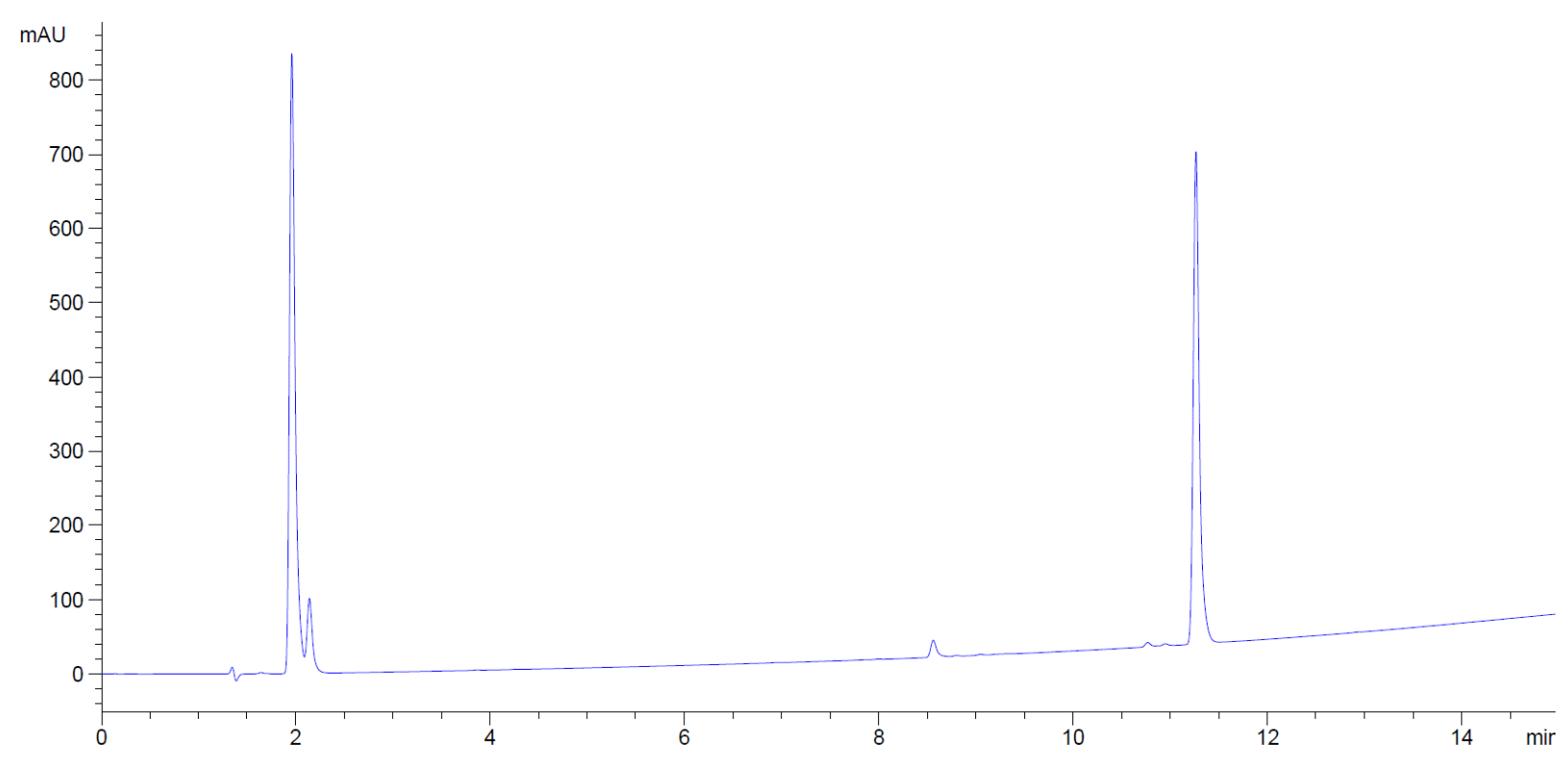

\section{$120 \mathrm{~min}$}

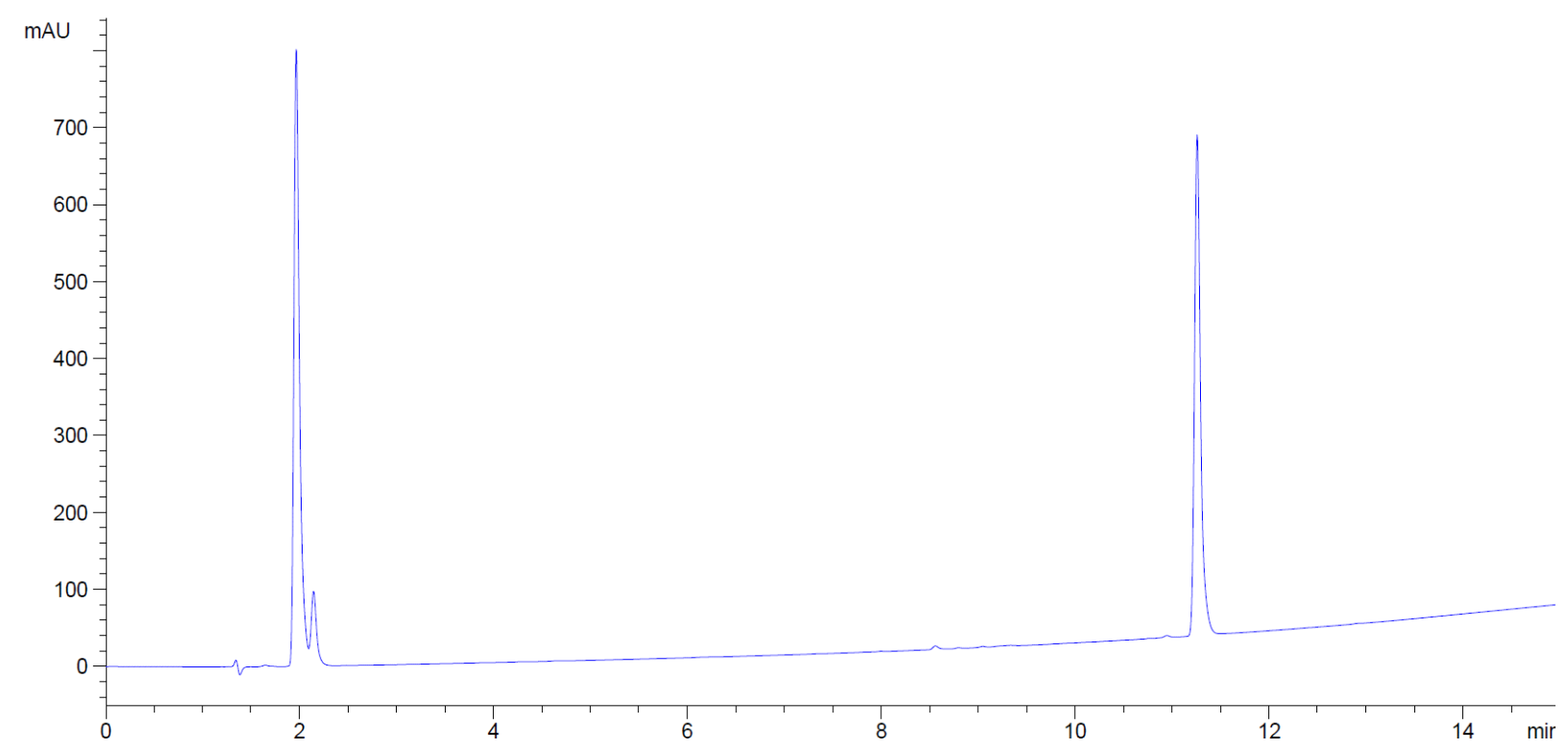

HPLC-S5. Fmoc-Arg(Pbf)-OH : DIC : OxymaPure [1:1:1], at RT, in DMF, in presence of nucleophile (tripeptidyl resin), $0.075 \mathrm{M}$. 


\section{0 min}

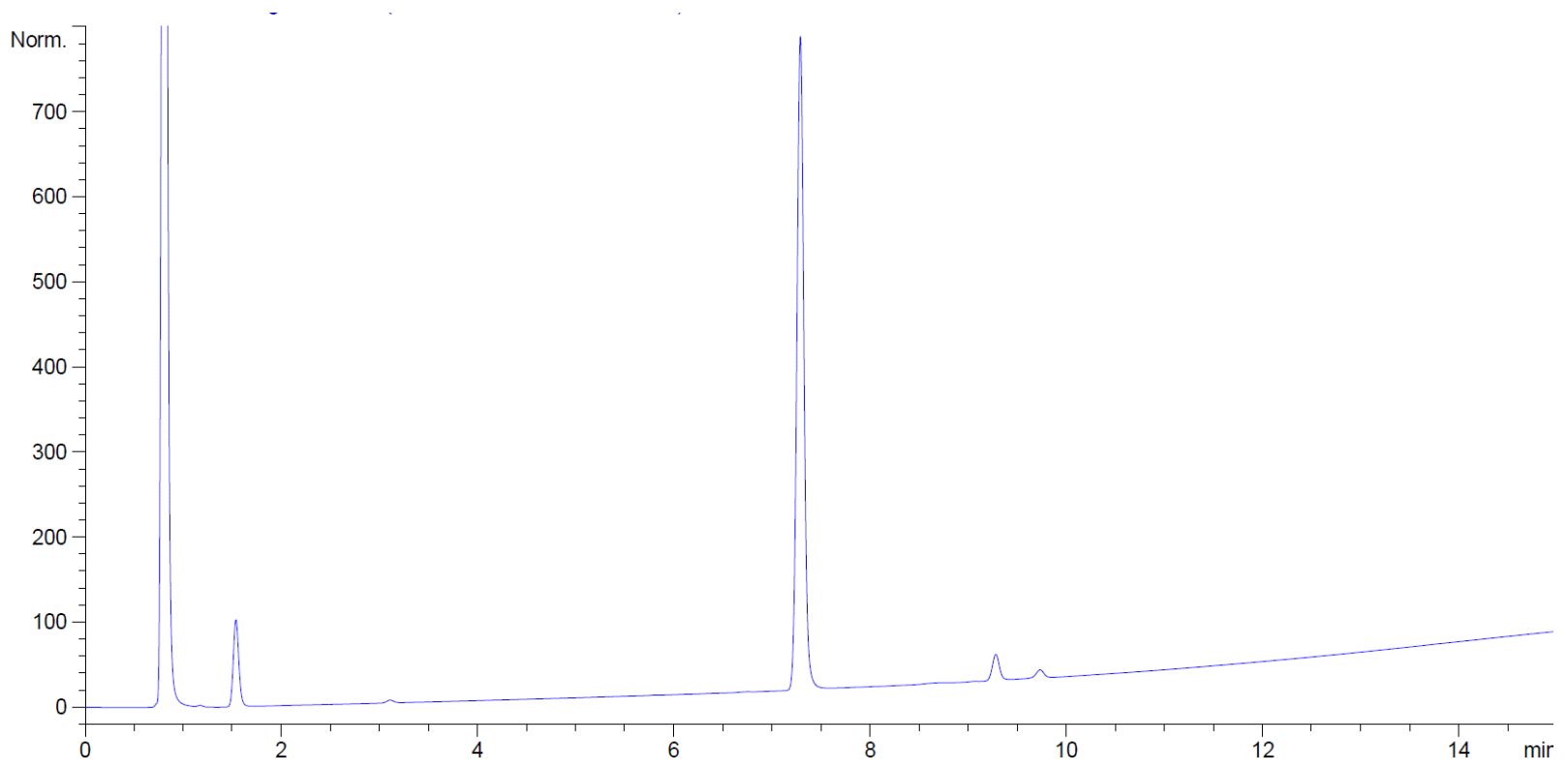

$30 \mathrm{~min}$

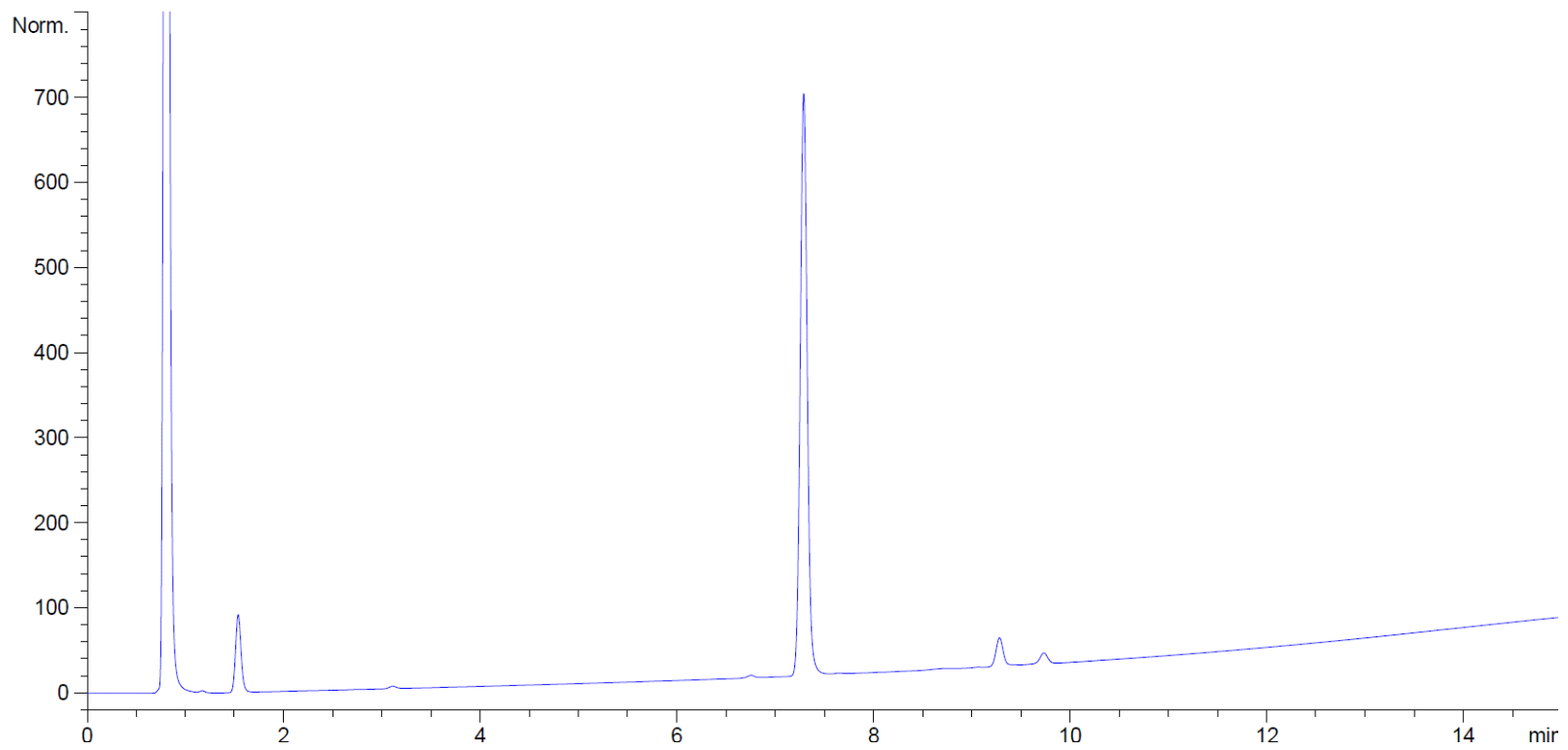

$60 \mathrm{~min}$ 


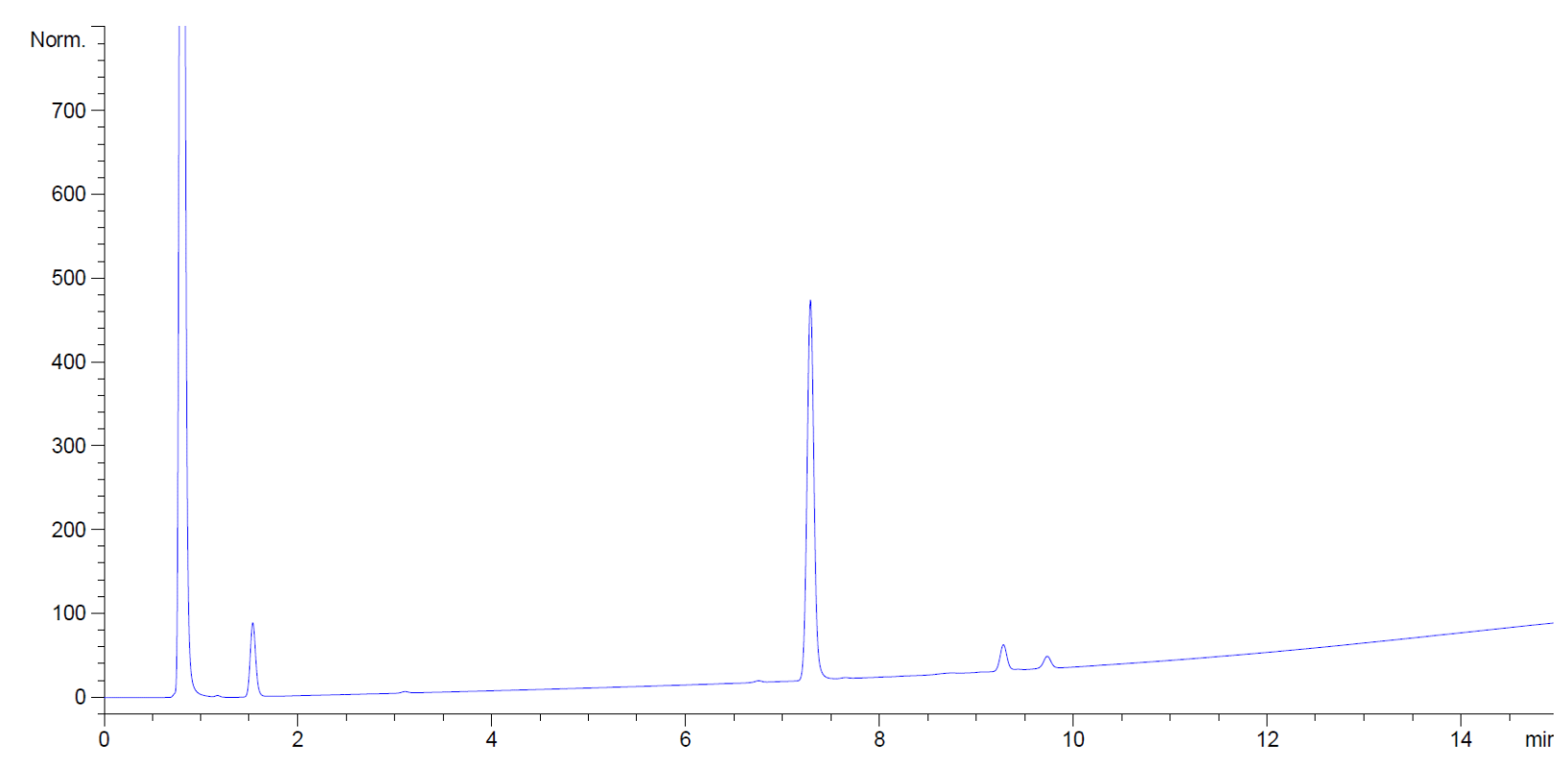

$120 \mathrm{~min}$

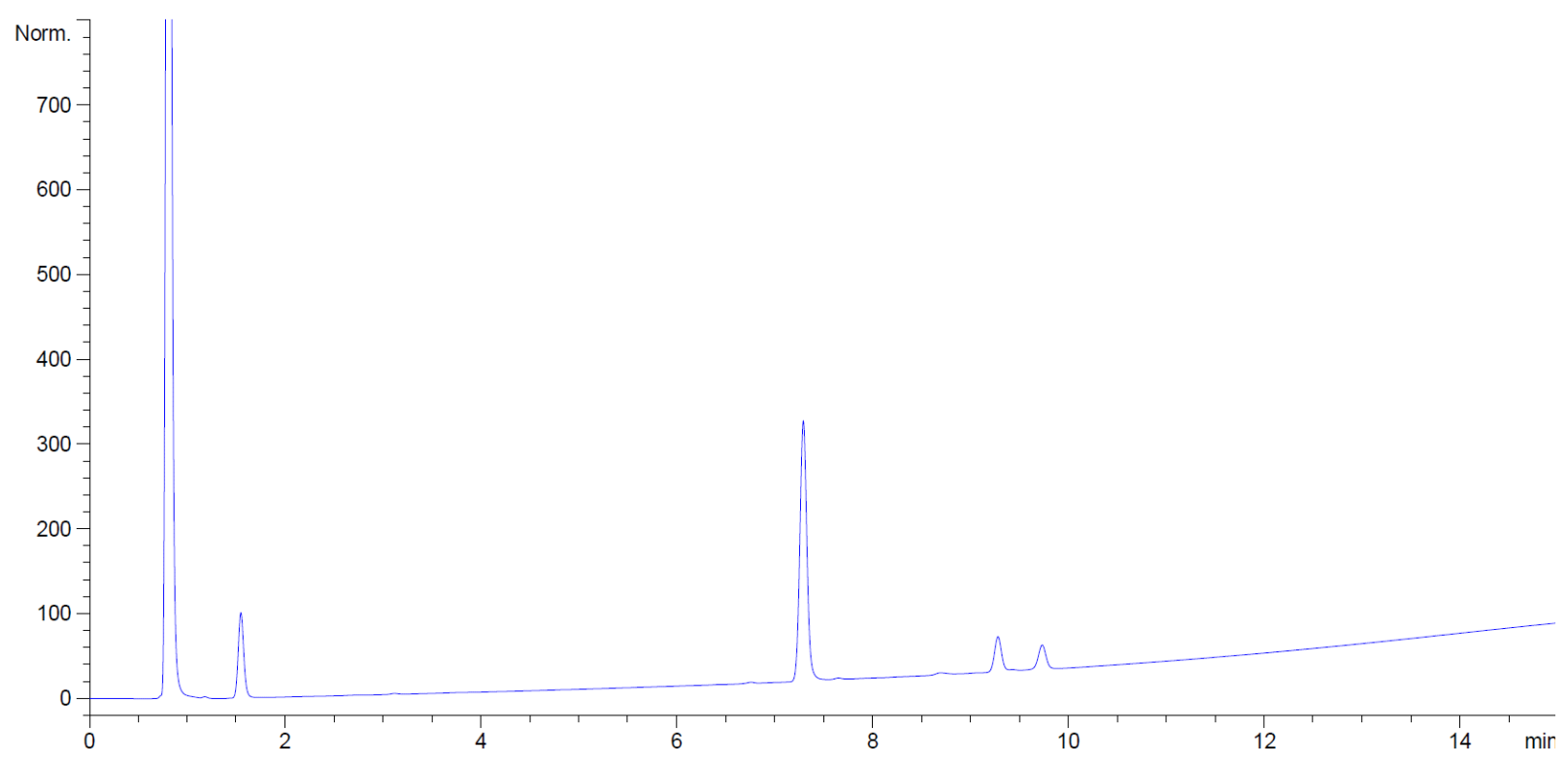

HPLC-S6. Fmoc-Arg(Pbf)-OH : DIC : OxymaPure [1:1:1], at RT, in NBP, in presence of nucleophile (tripeptidyl resin), 0.075M. 


\section{0 min}

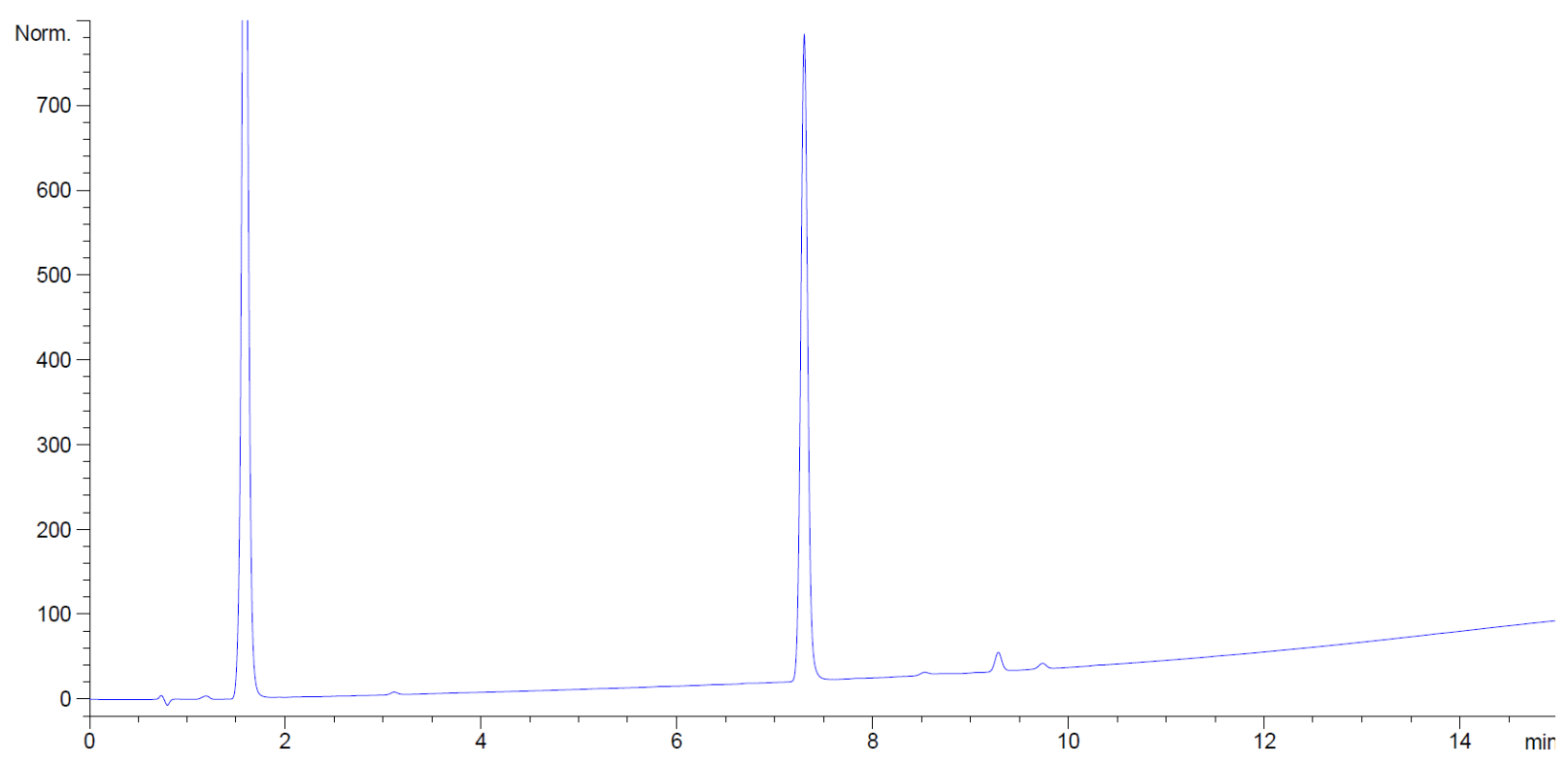

$30 \mathrm{~min}$

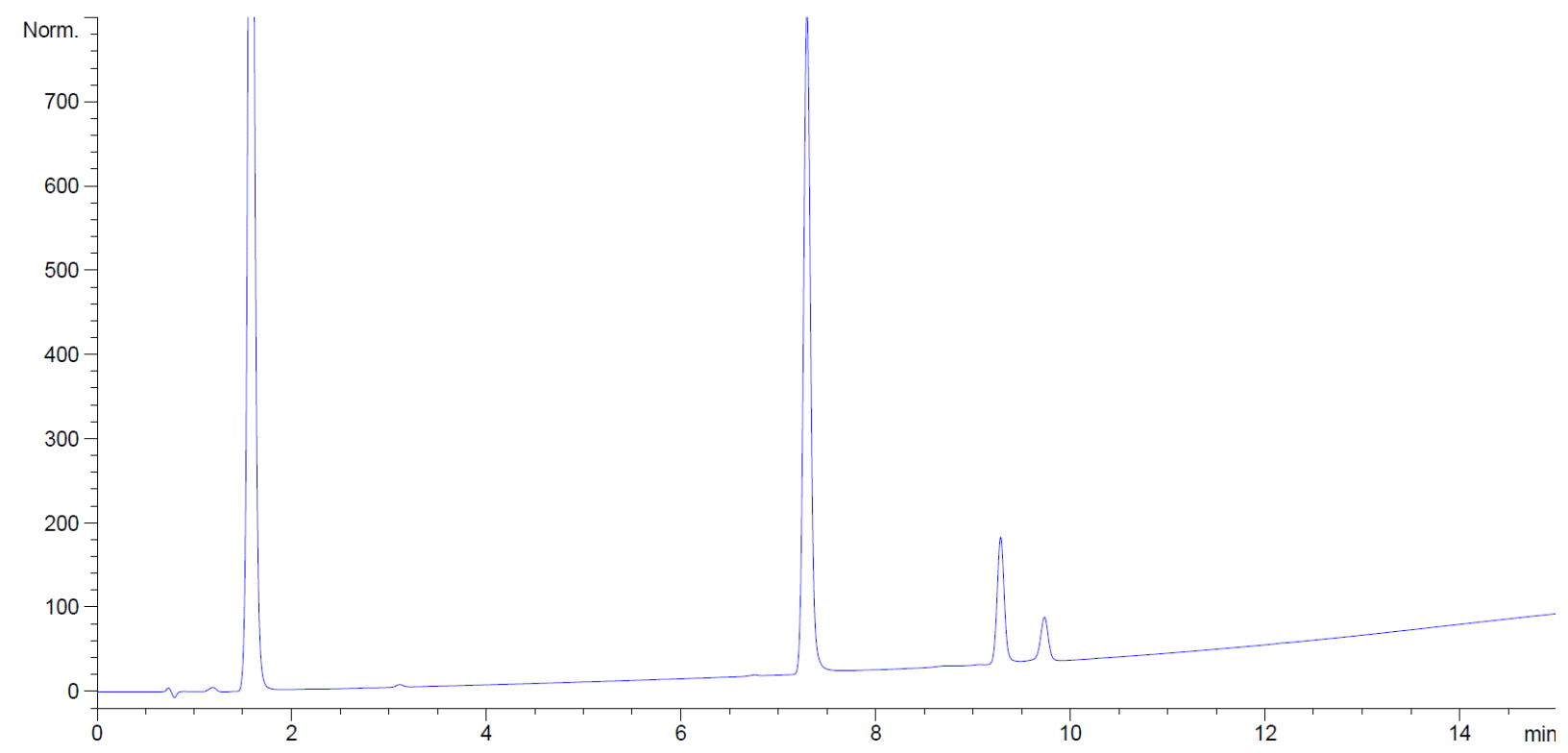

$60 \mathrm{~min}$ 


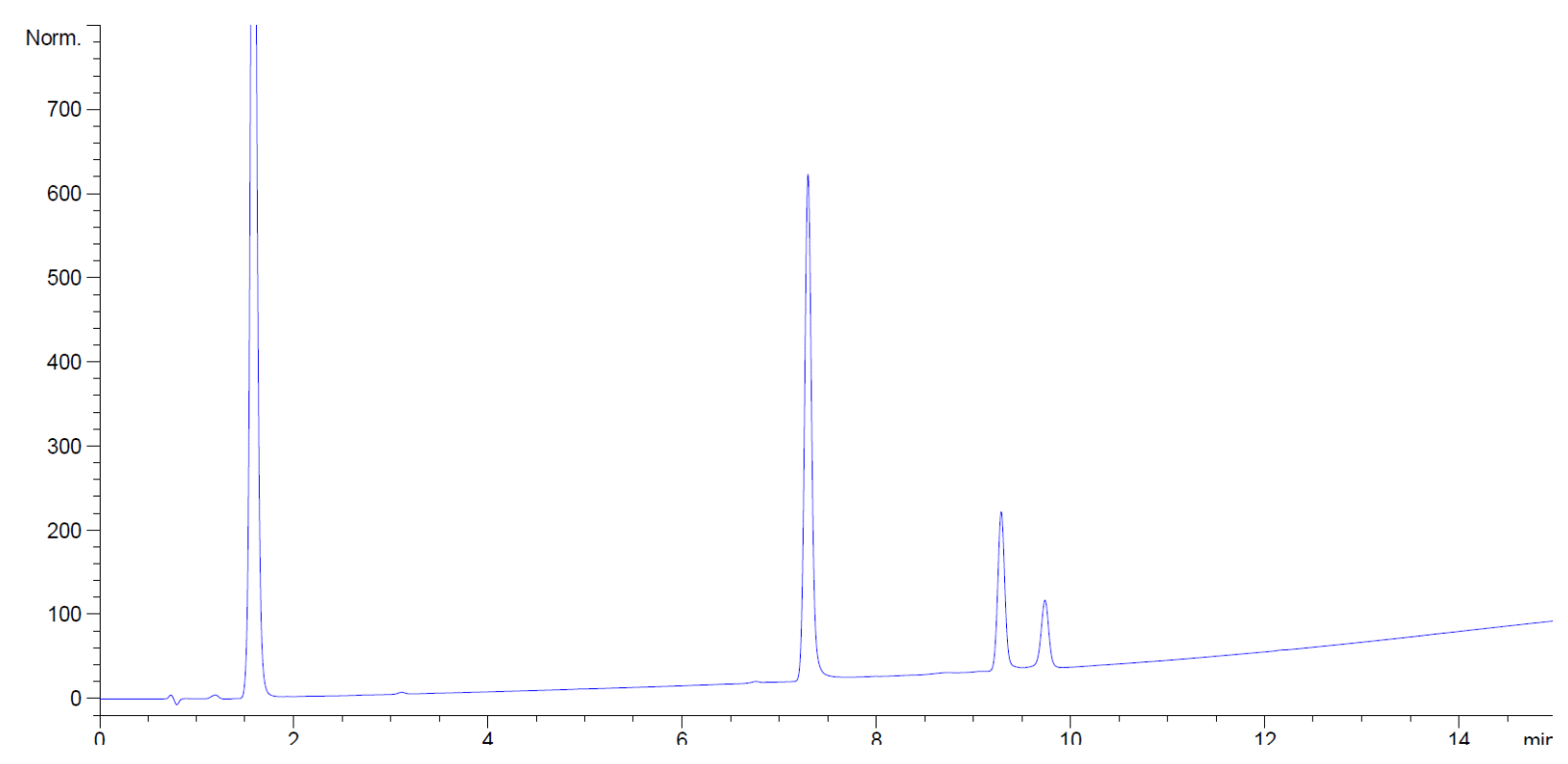

$120 \mathrm{~min}$

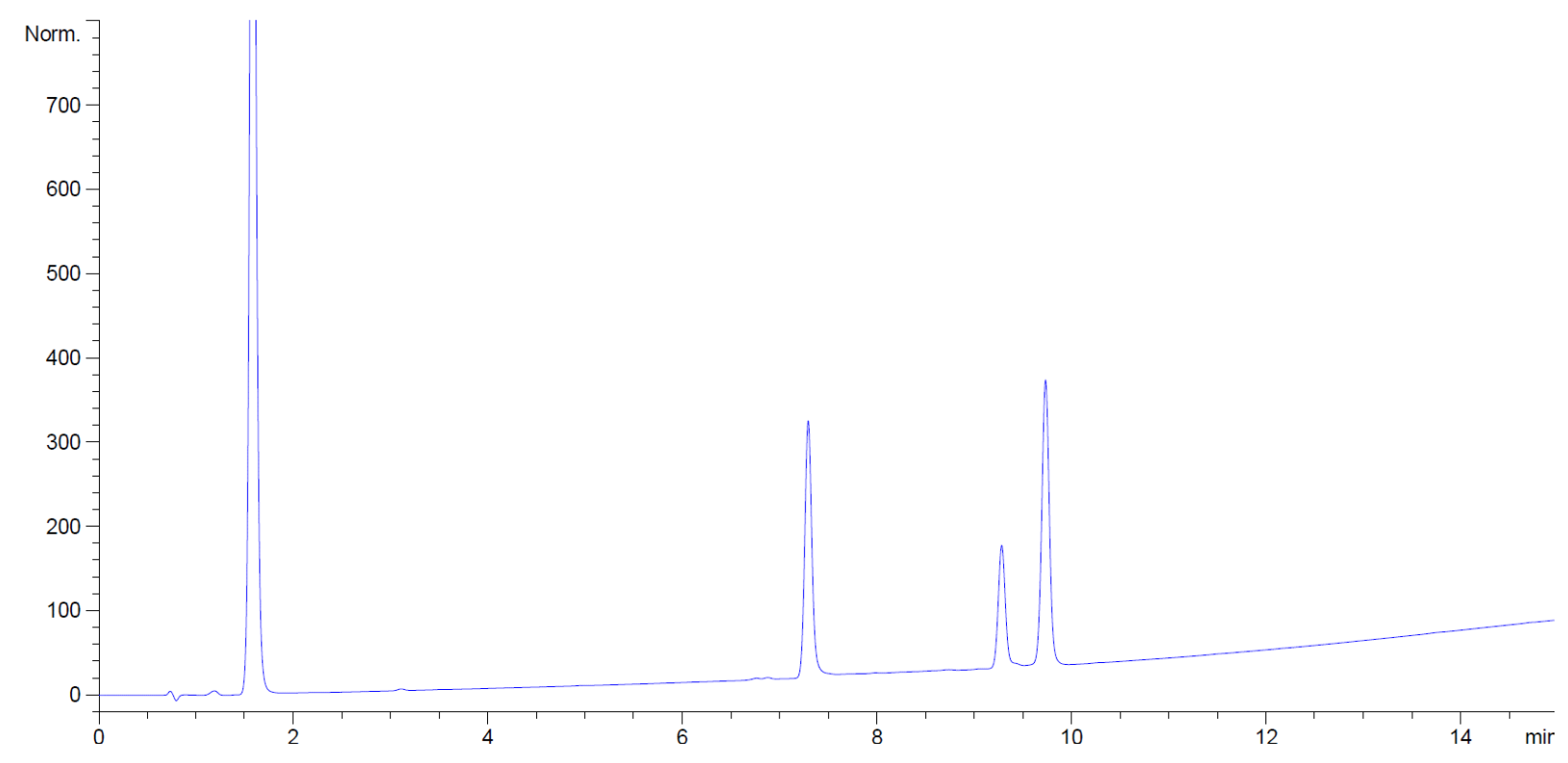

HPLC-S7. Fmoc-Arg(Pbf)-OH : DIC : OxymaPure [1:1:1], at $45^{\circ} \mathrm{C}$, in DMF, in presence of nucleophile (tripeptidyl resin), 0.075M 


\section{0 min}

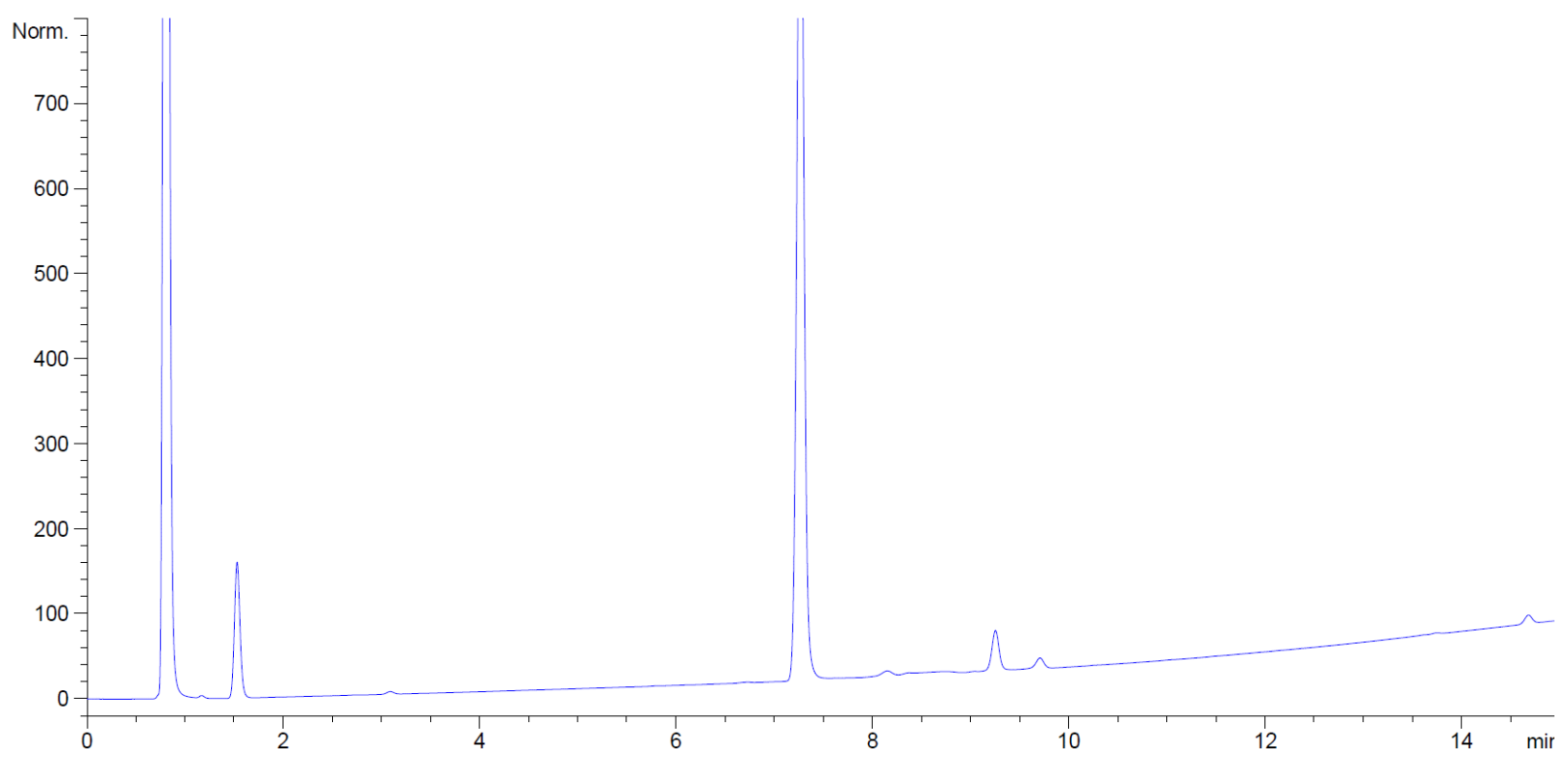

$30 \mathrm{~min}$

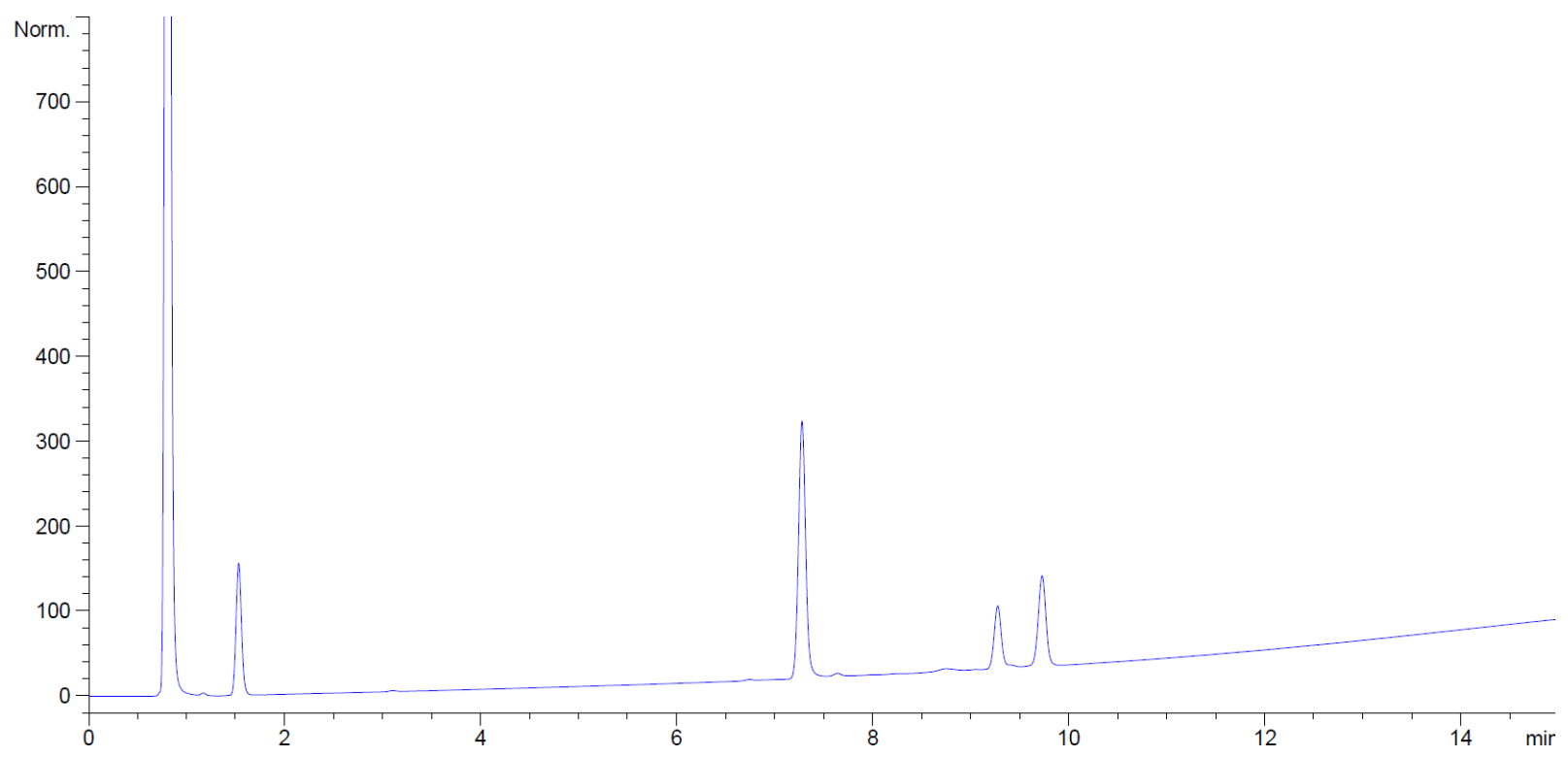

$60 \mathrm{~min}$ 


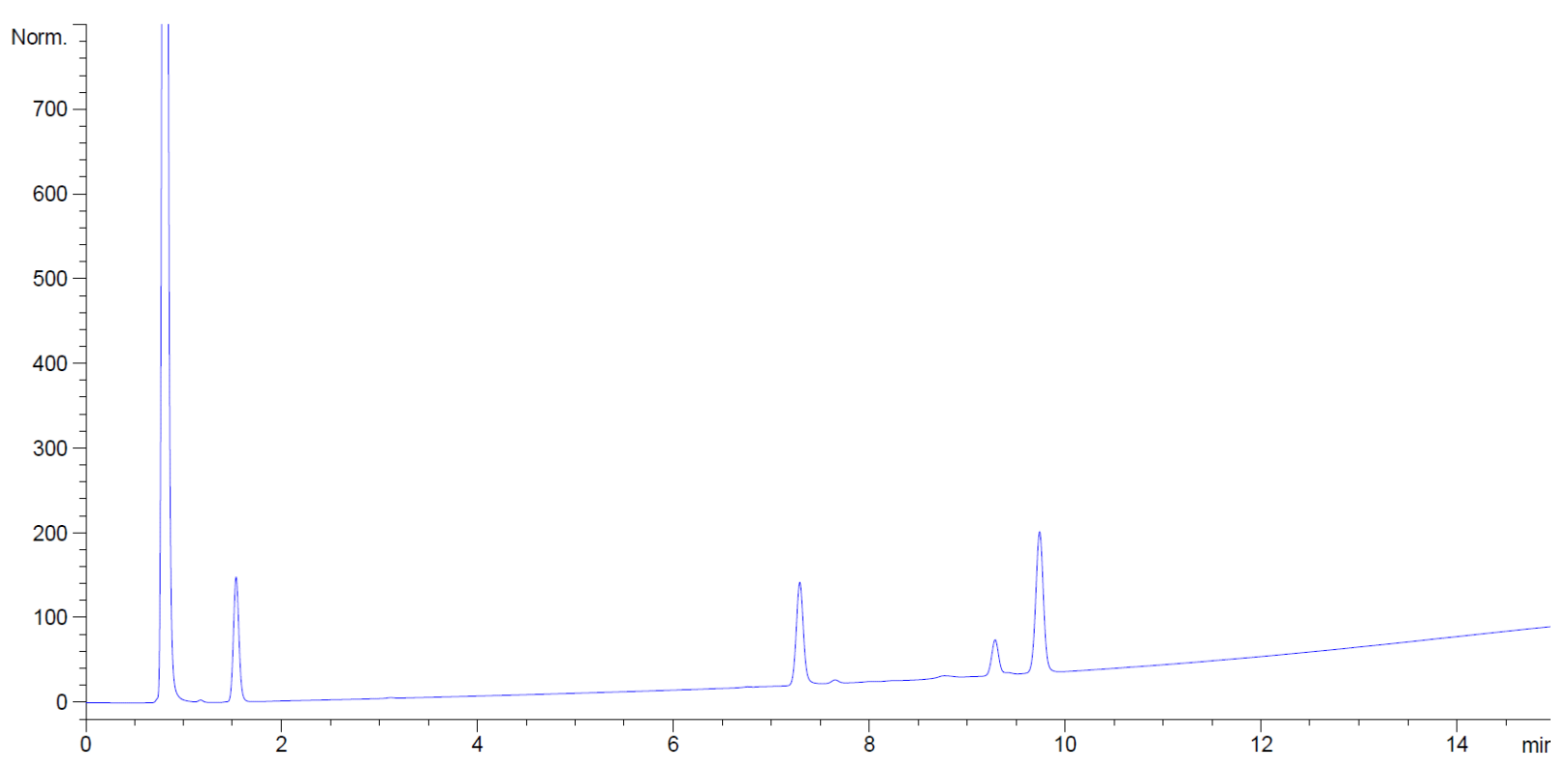

$120 \mathrm{~min}$

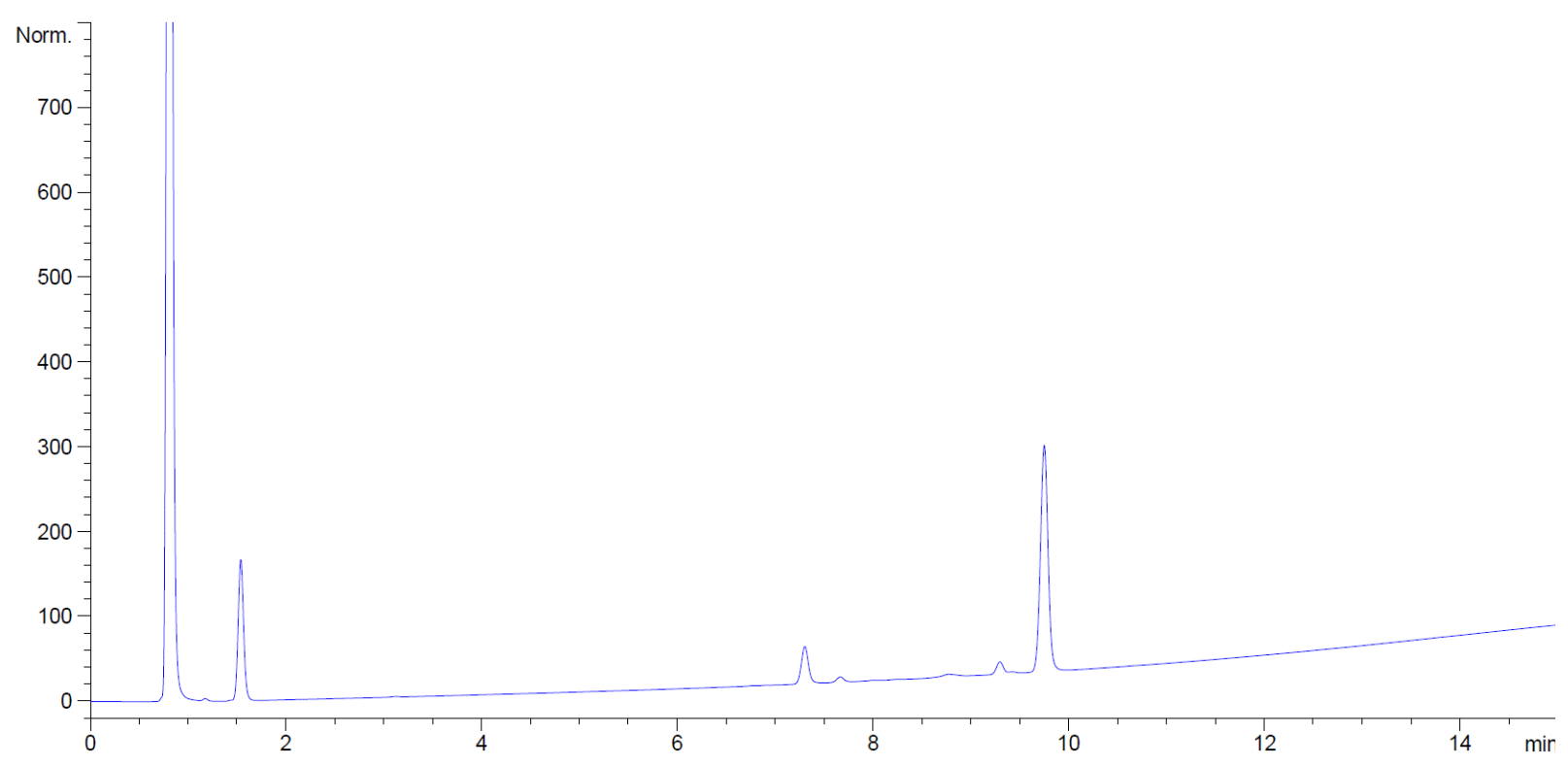

HPLC-S8. Fmoc-Arg(Pbf)-OH : DIC : OxymaPure [1:1:1], at $45^{\circ} \mathrm{C}$, in NBP, in presence of nucleophile (tripeptidyl resin), 0.075M. 


\section{0 min}

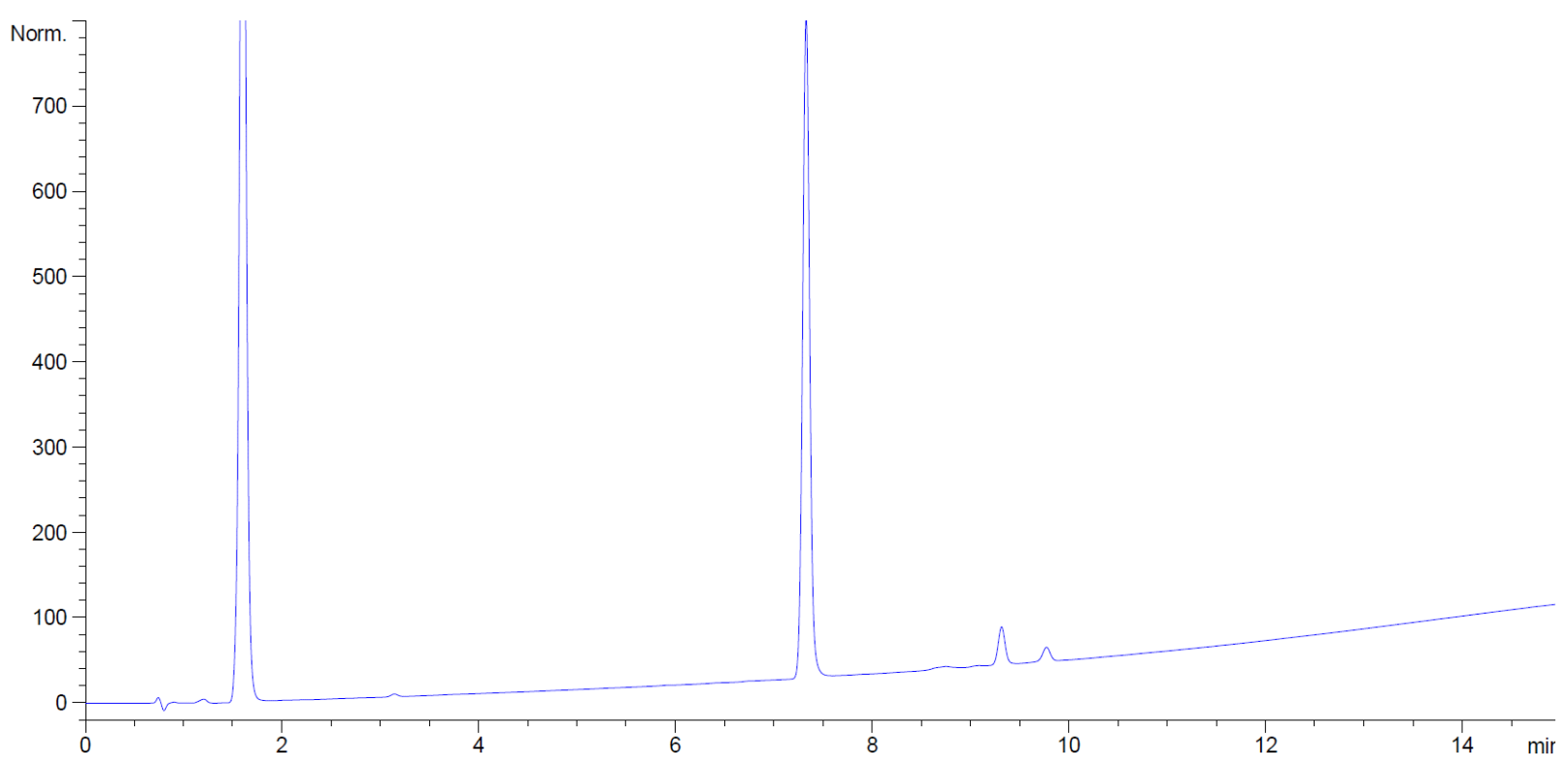

$30 \mathrm{~min}$

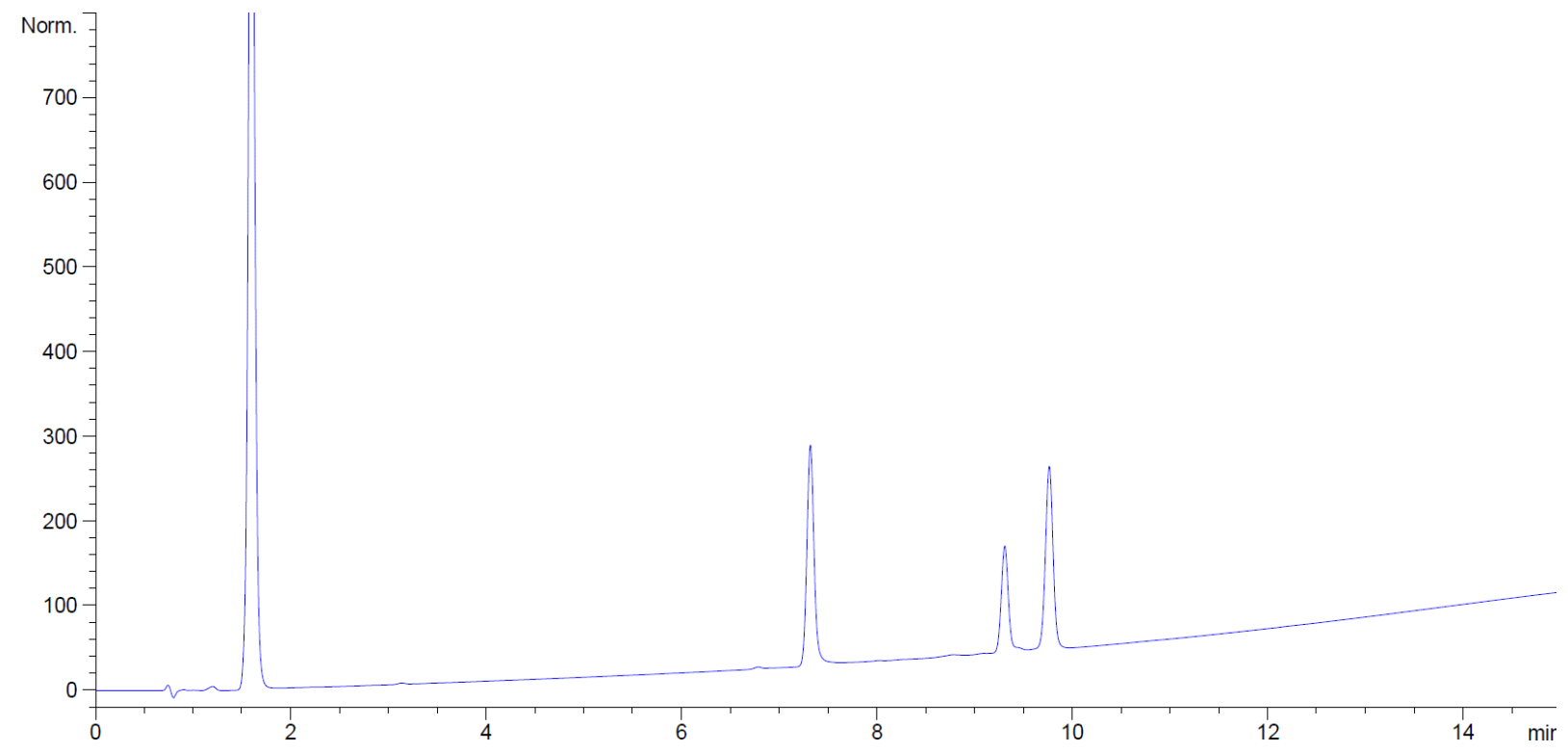

$60 \mathrm{~min}$ 


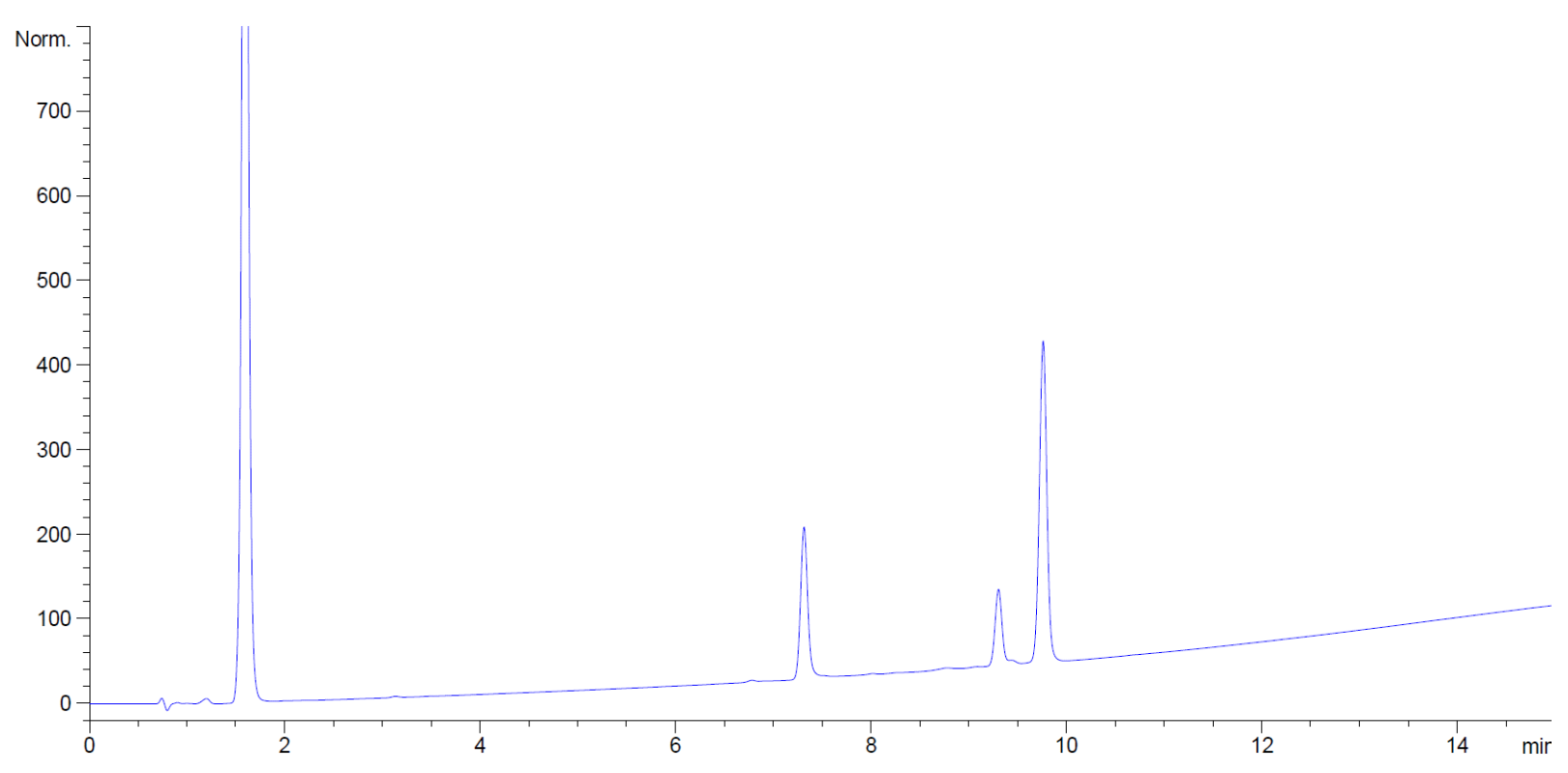

$120 \mathrm{~min}$

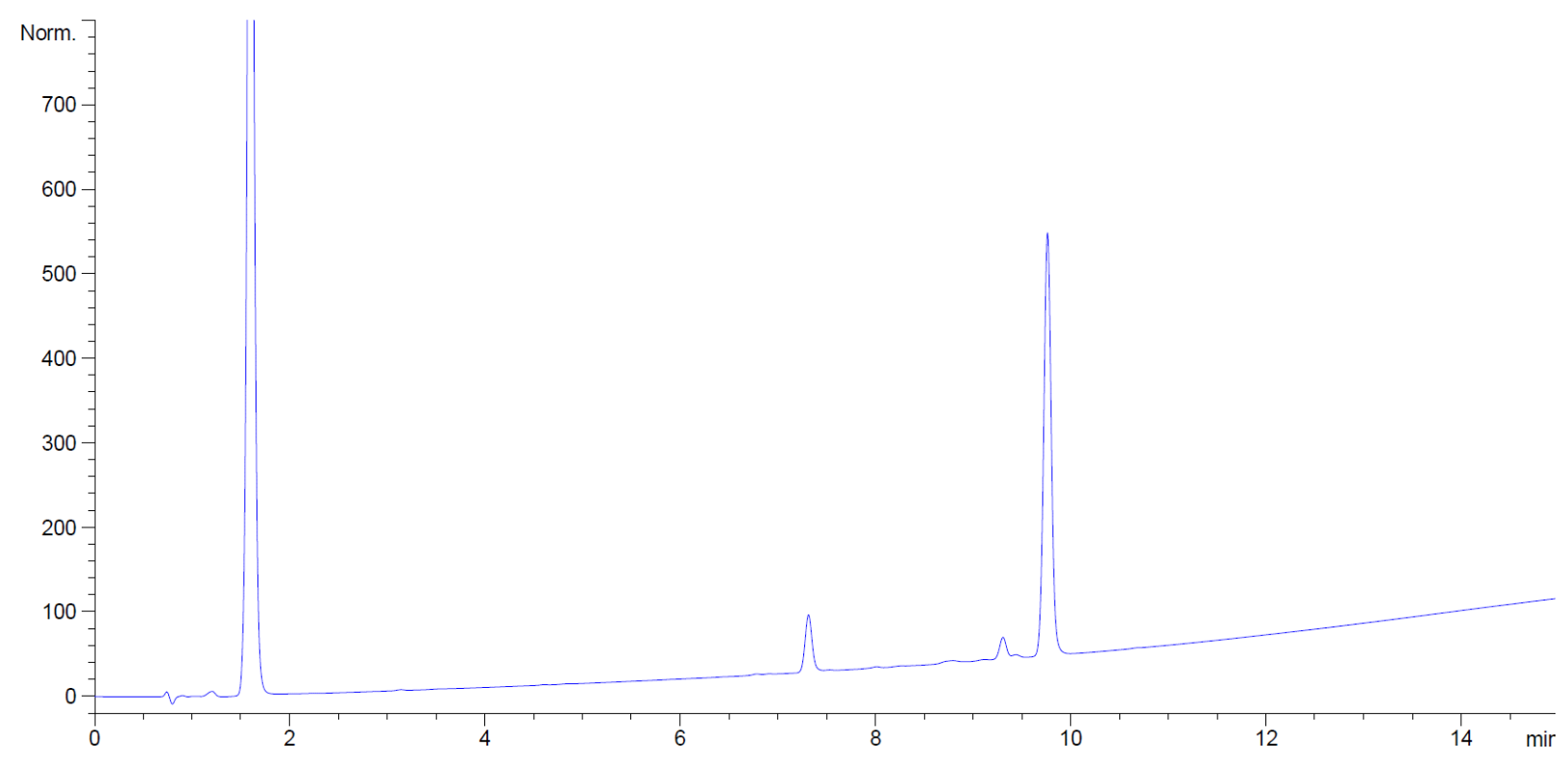

HPLC-S9. Fmoc-Arg(Pbf)-OH : DIC : OxymaPure [1:1:1], at $60^{\circ} \mathrm{C}$, in NBP, in presence of nucleophile (tripeptidyl resin), 0.075M. 


\section{0 min}

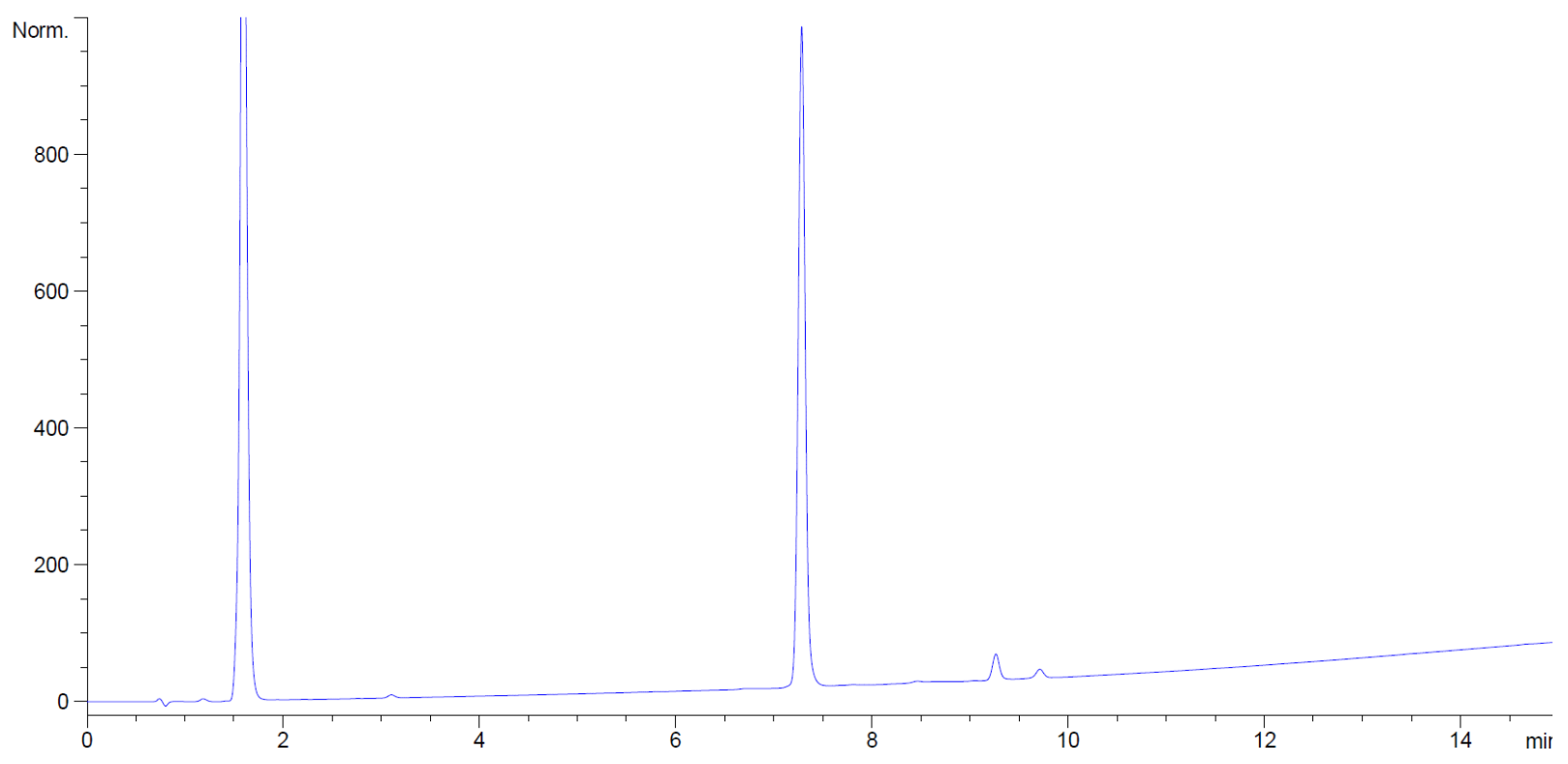

$30 \mathrm{~min}$

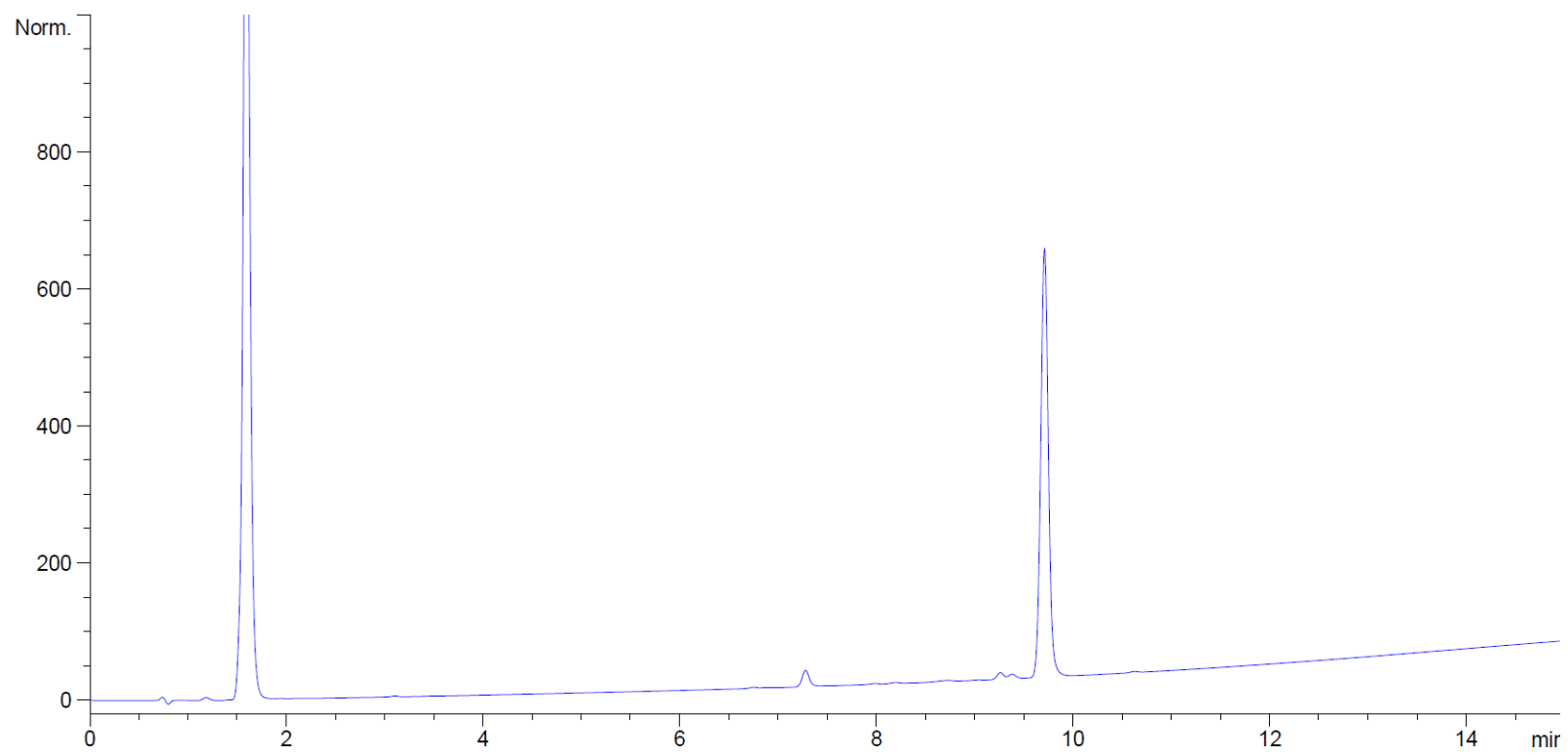

$60 \mathrm{~min}$ 


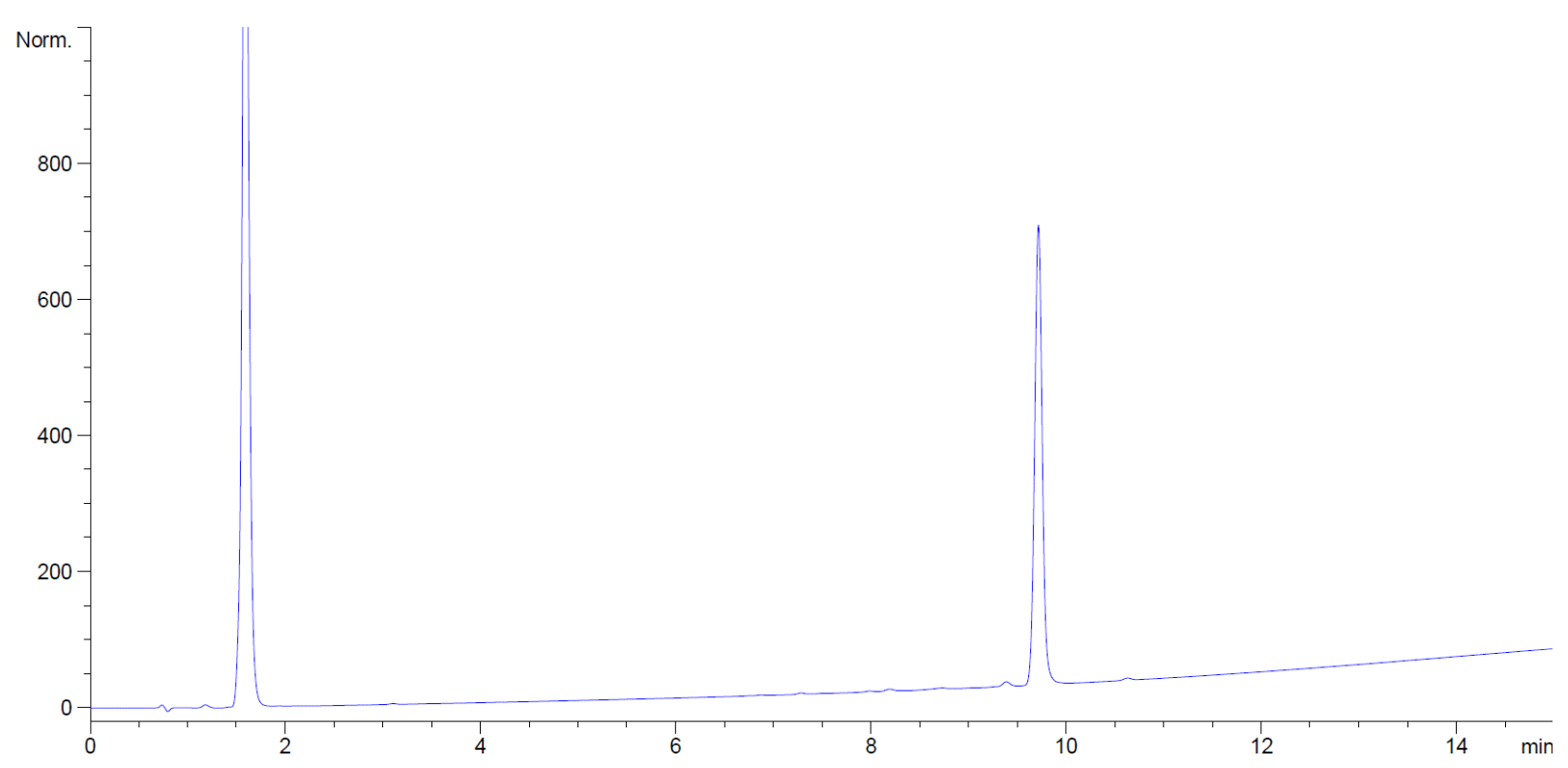

$120 \mathrm{~min}$

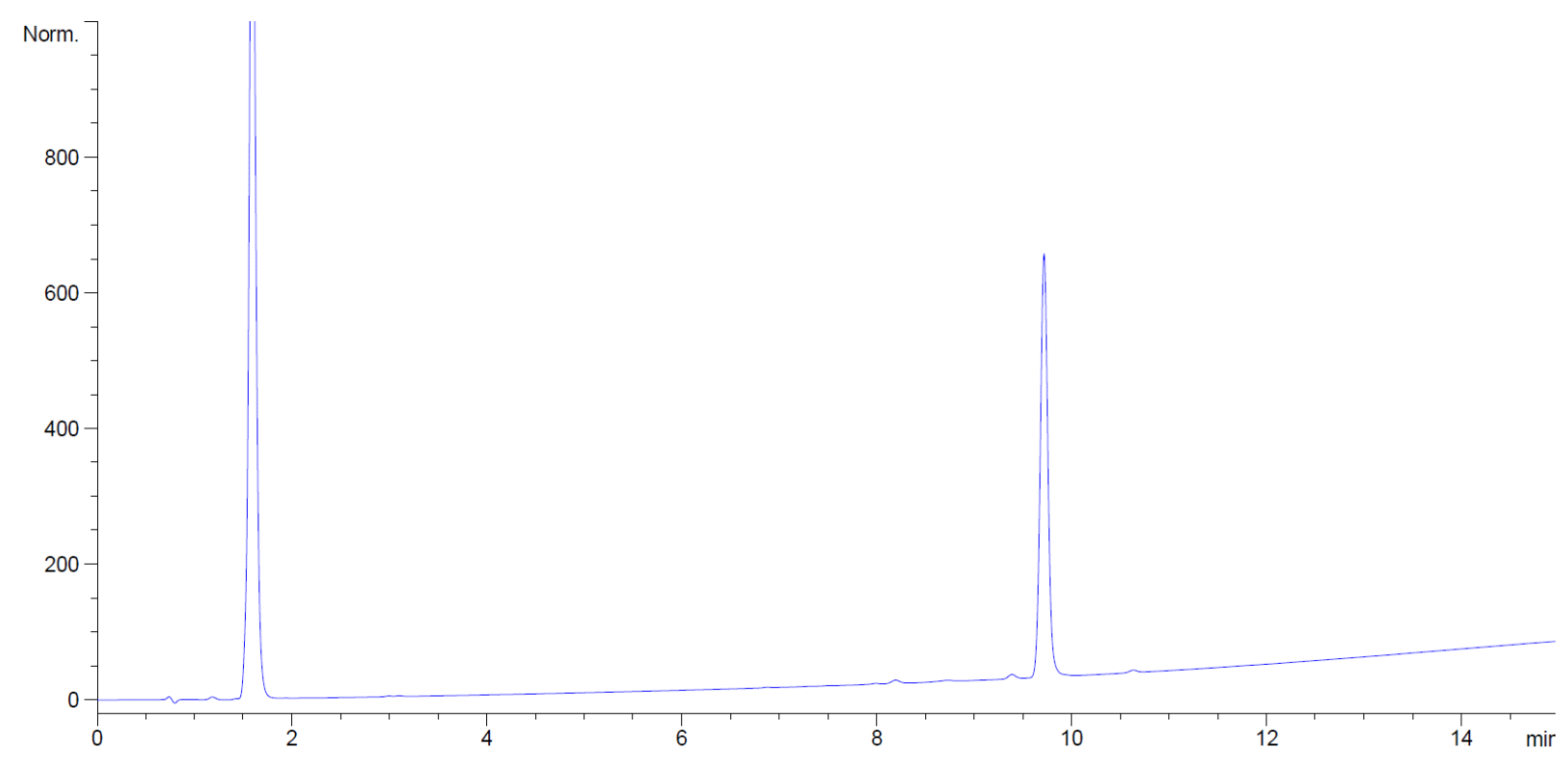

HPLC-S10. Fmoc-Arg(Pbf)-OH : DIC : OxymaPure [1:1:1], at $45^{\circ} \mathrm{C}$, in NBP, in presence of nucleophile (tripeptidyl resin), Pre-conditioned of tripeptidyl resin, $0.075 \mathrm{M}$. 
0 min

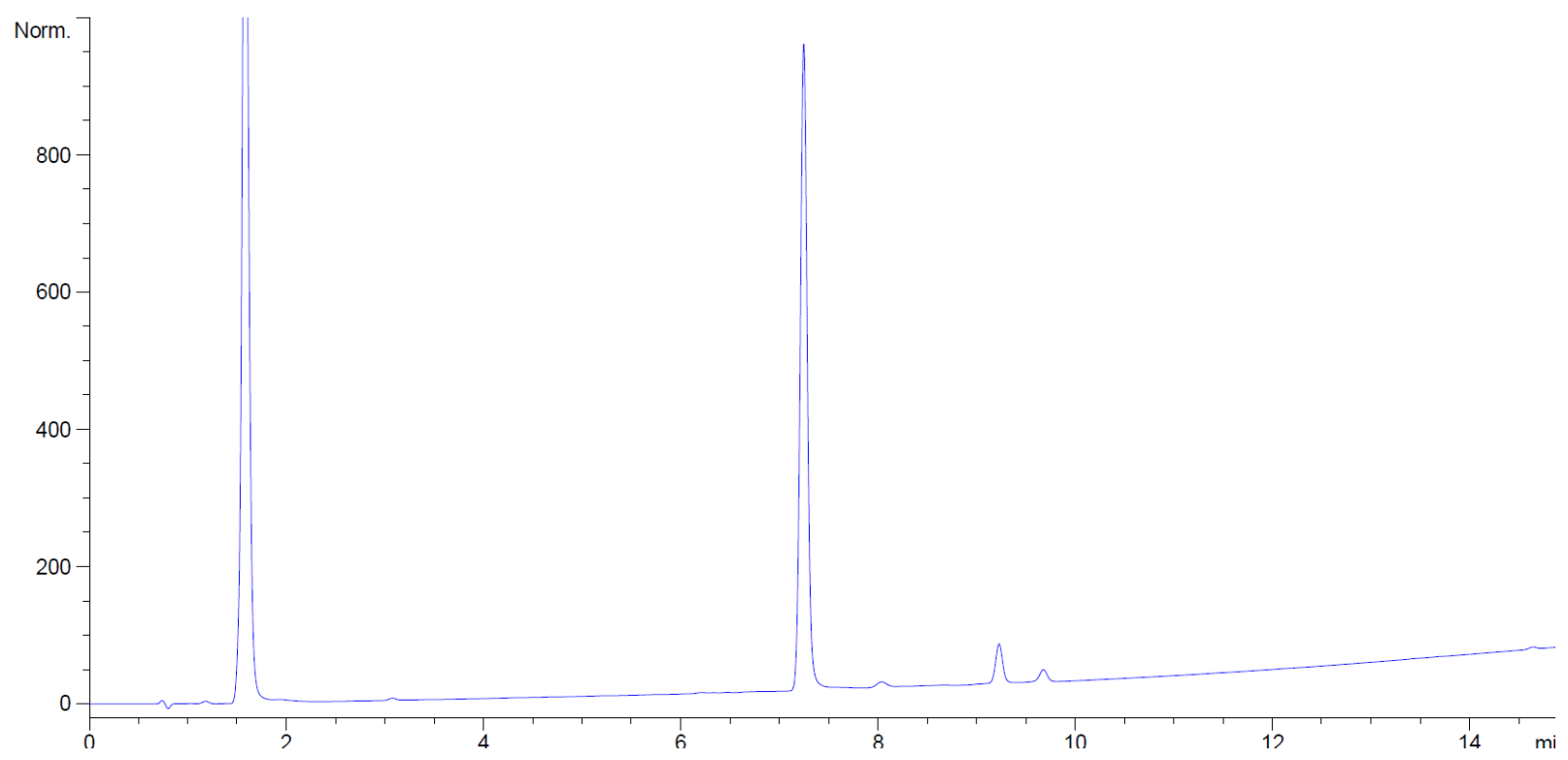

$30 \mathrm{~min}$

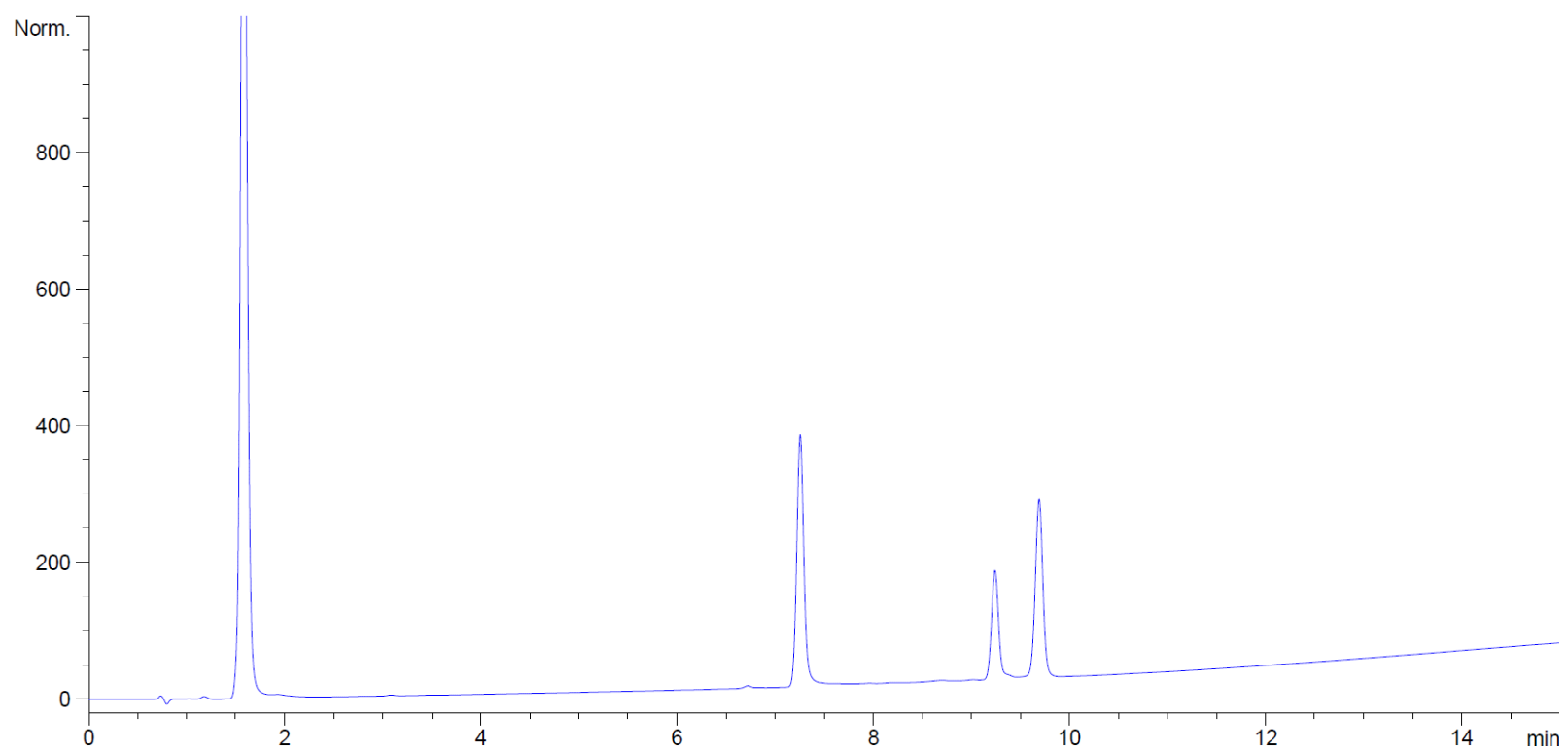

$60 \mathrm{~min}$ 


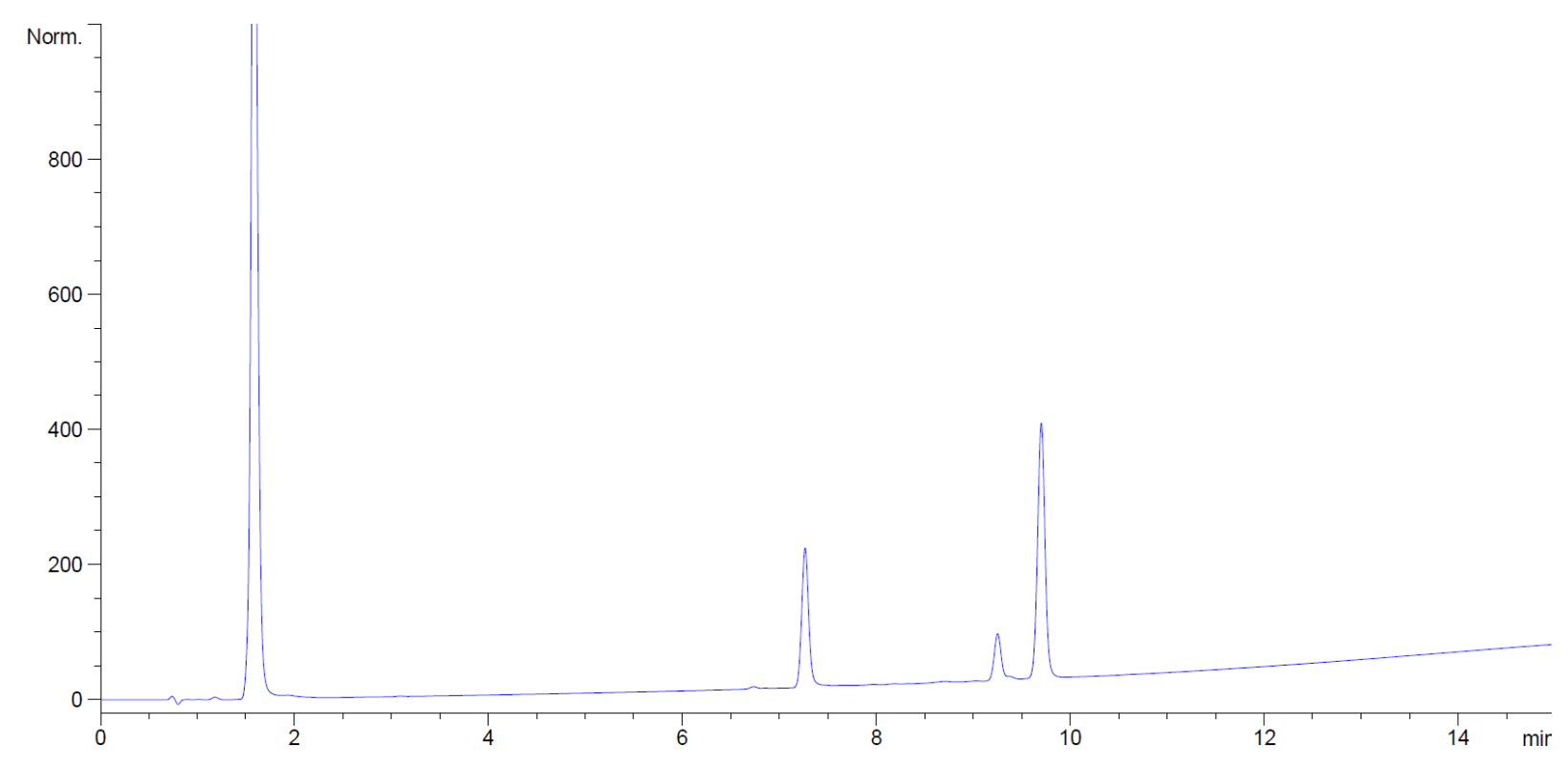

$120 \mathrm{~min}$

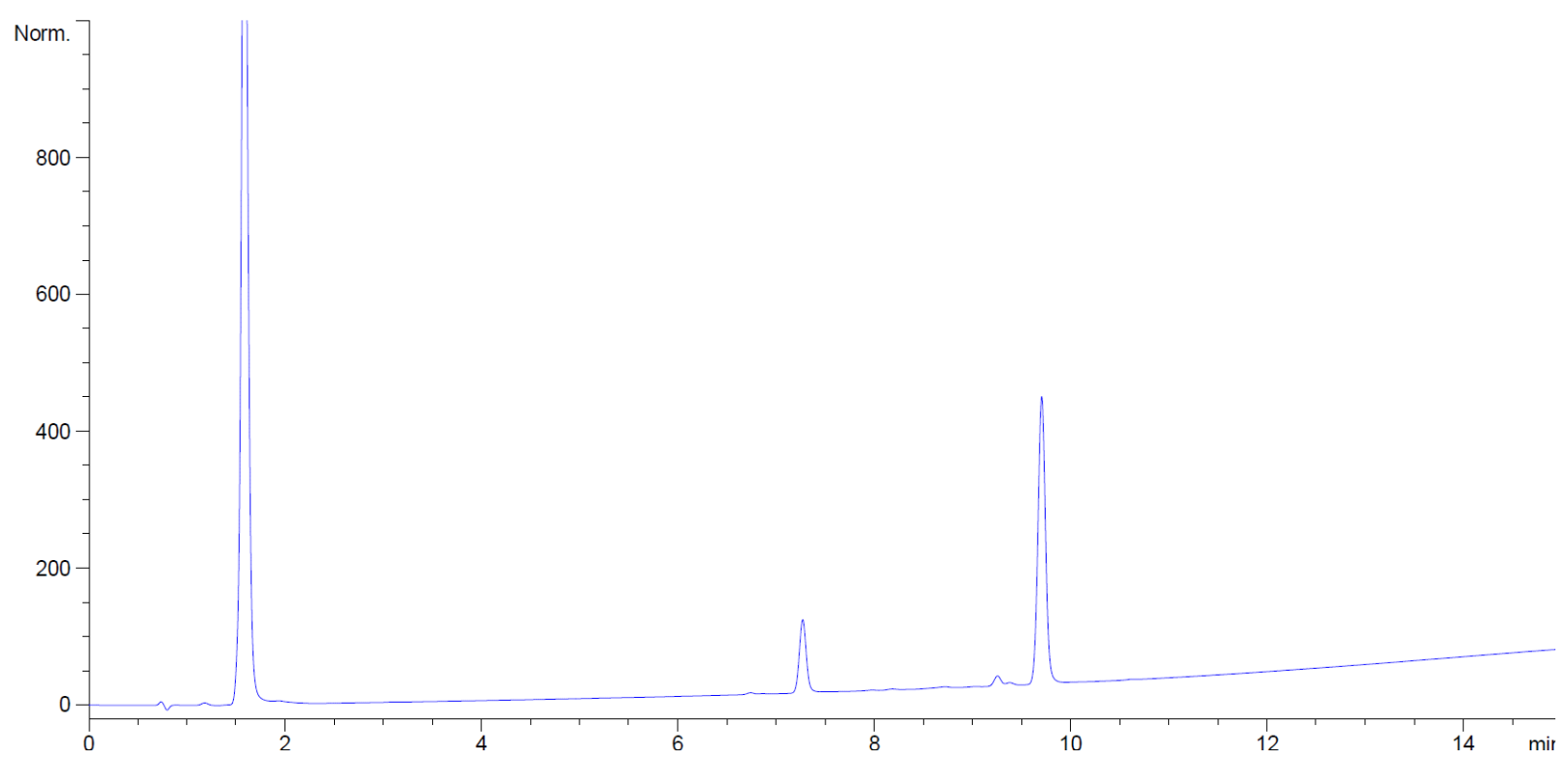

HPLC-S11. Fmoc-Arg(Pbf)-OH : DIC : OxymaPure [1:1:1], at $45^{\circ} \mathrm{C}$, in NBP, in presence of nucleophile (tripeptidyl resin), In-situ activation, $0.15 \mathrm{M}$. 
0 min

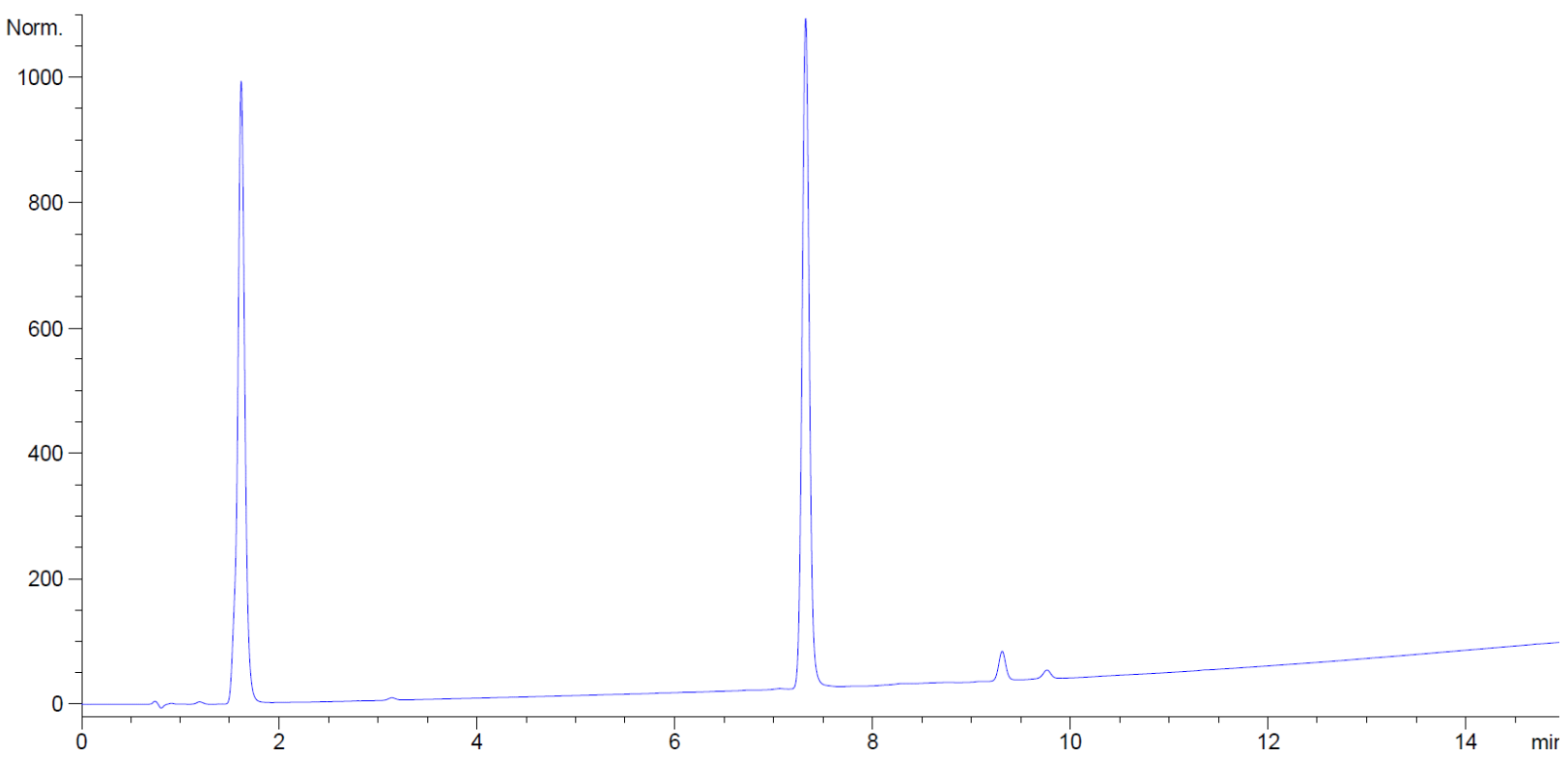

$30 \mathrm{~min}$

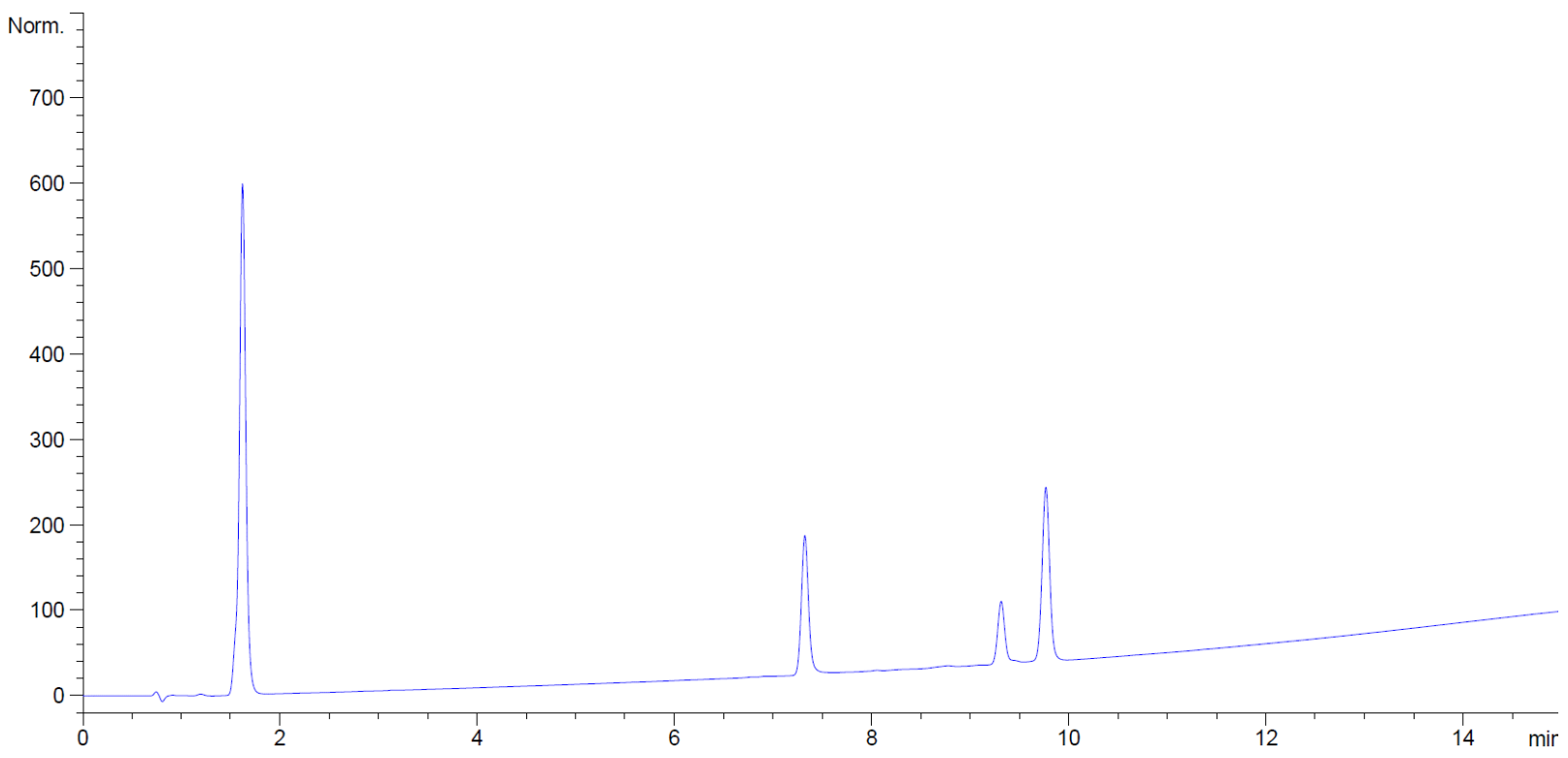

$60 \mathrm{~min}$ 


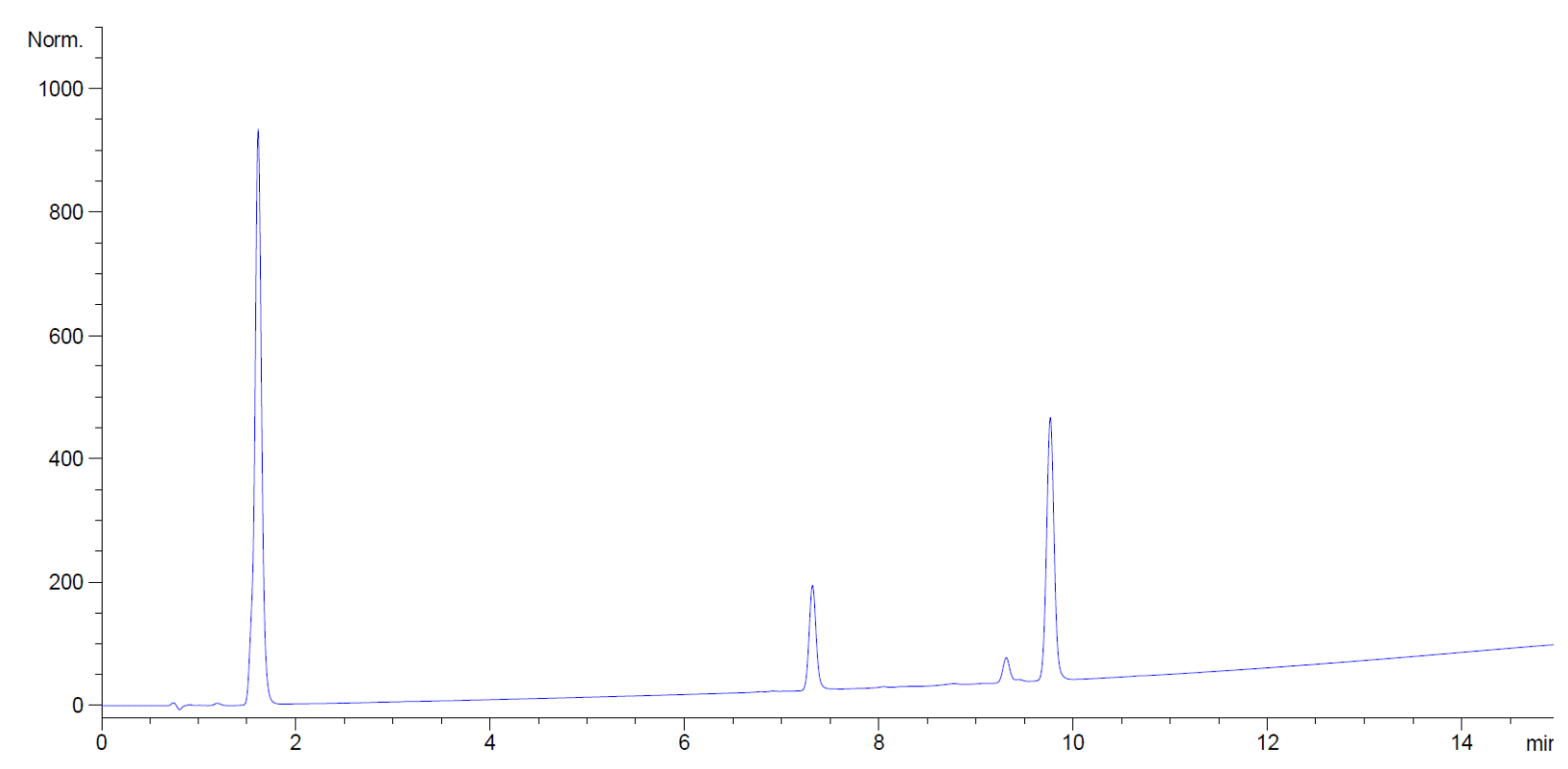

$120 \mathrm{~min}$

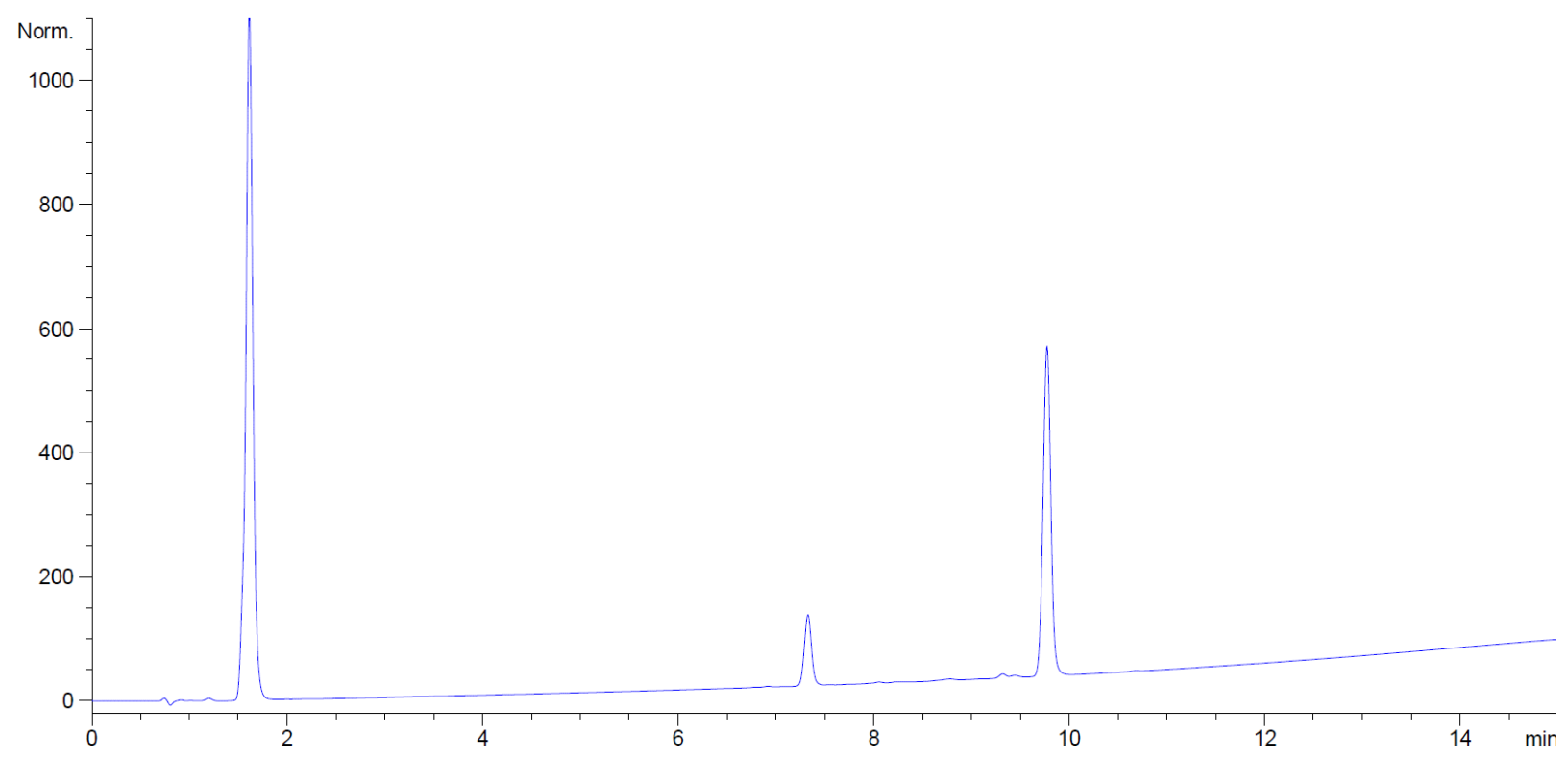

HPLC-S12. Fmoc-Arg(Pbf)-OH : DIC : OxymaPure [1:1:1], at $45^{\circ} \mathrm{C}$, in NBP, in presence of nucleophile (tripeptidyl resin), In-situ activation, $0.075 \mathrm{M}$. 
0 min

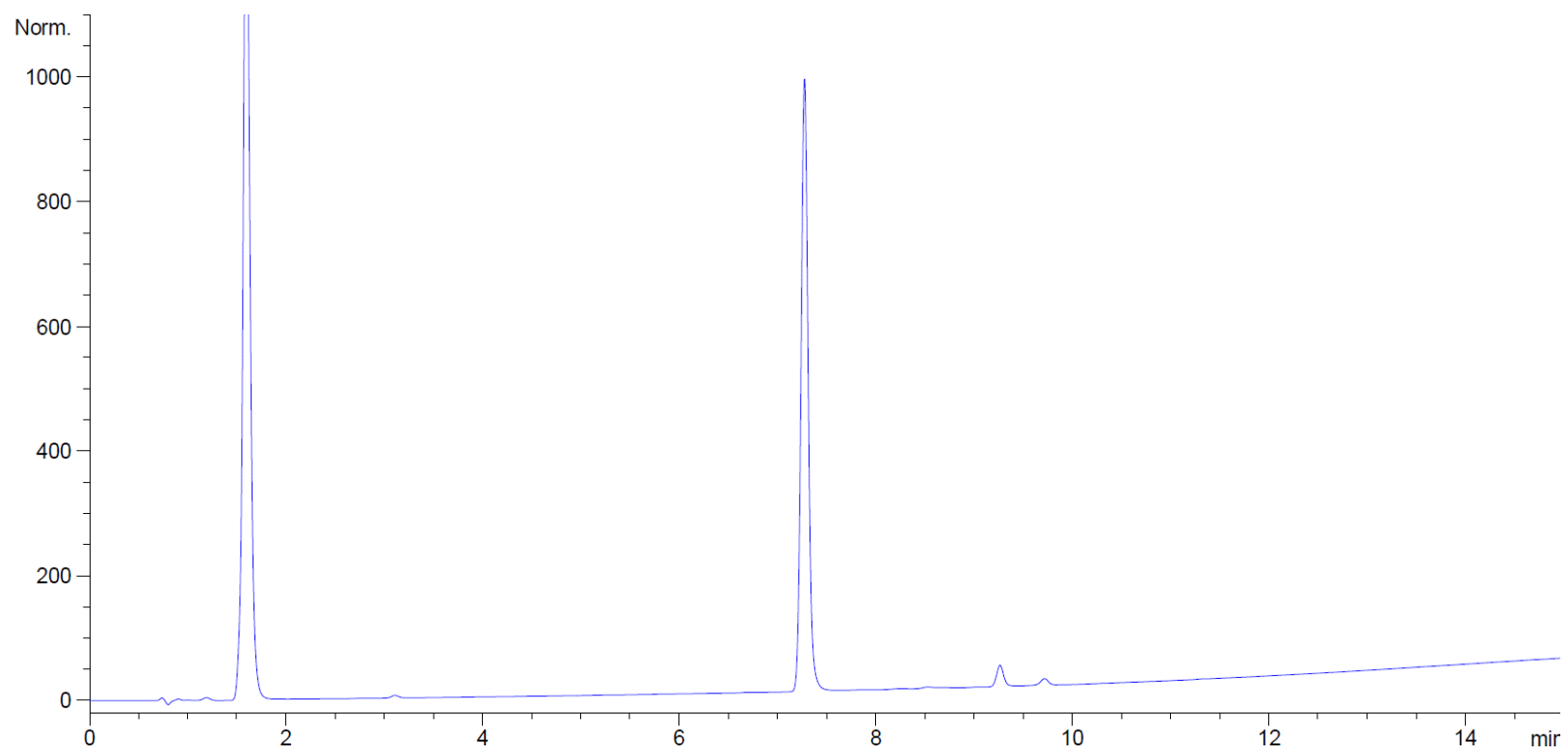

$30 \mathrm{~min}$

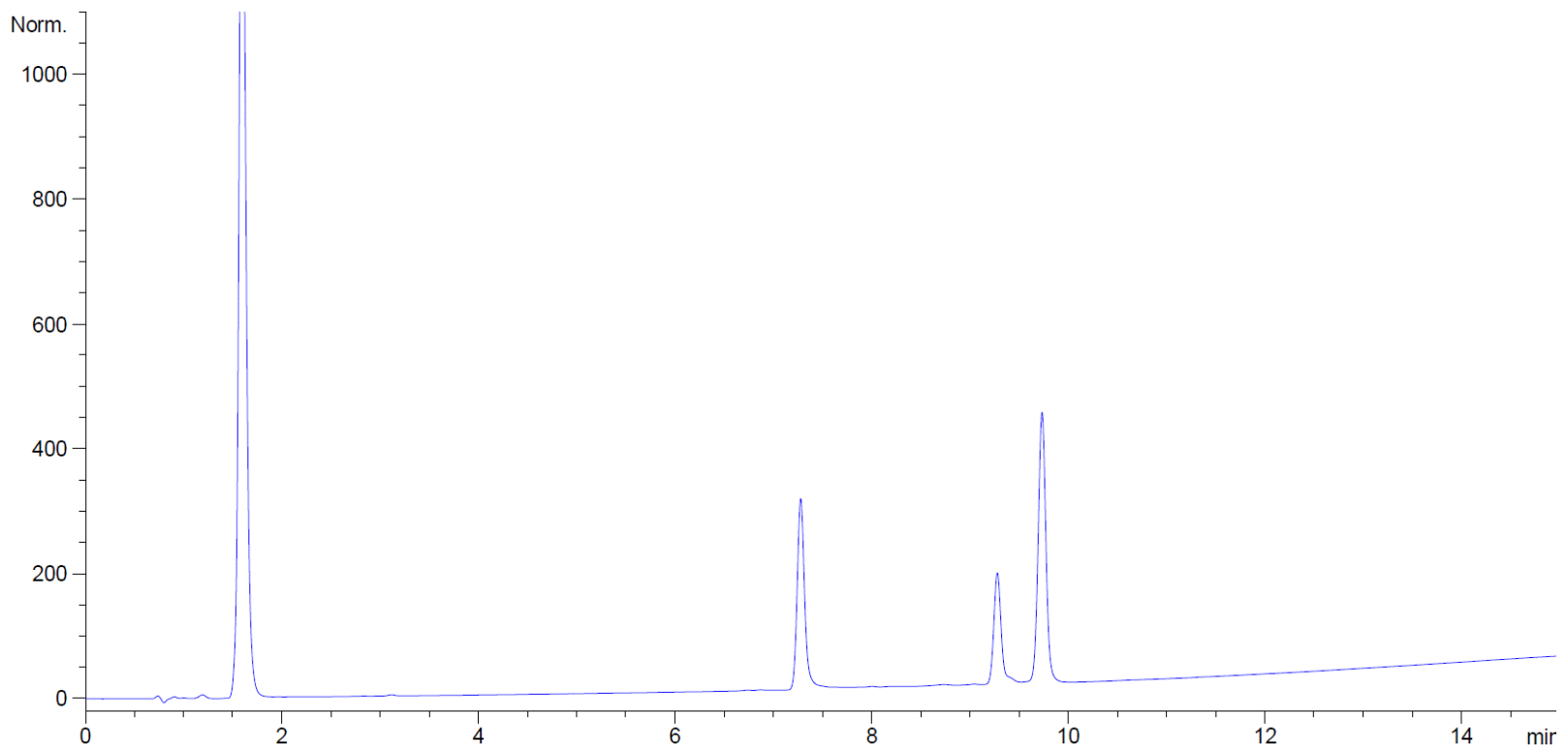

60 min 


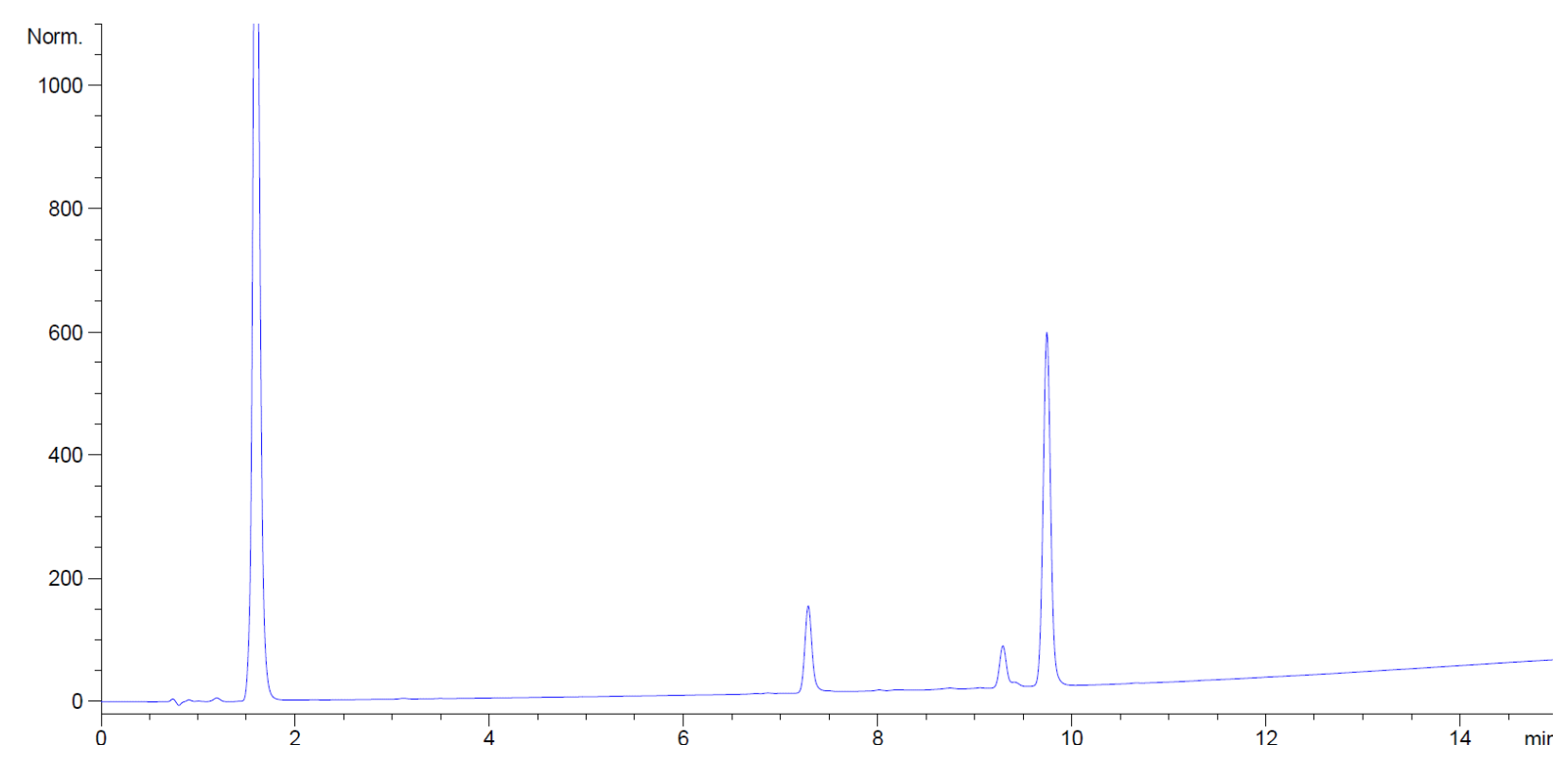

$120 \mathrm{~min}$

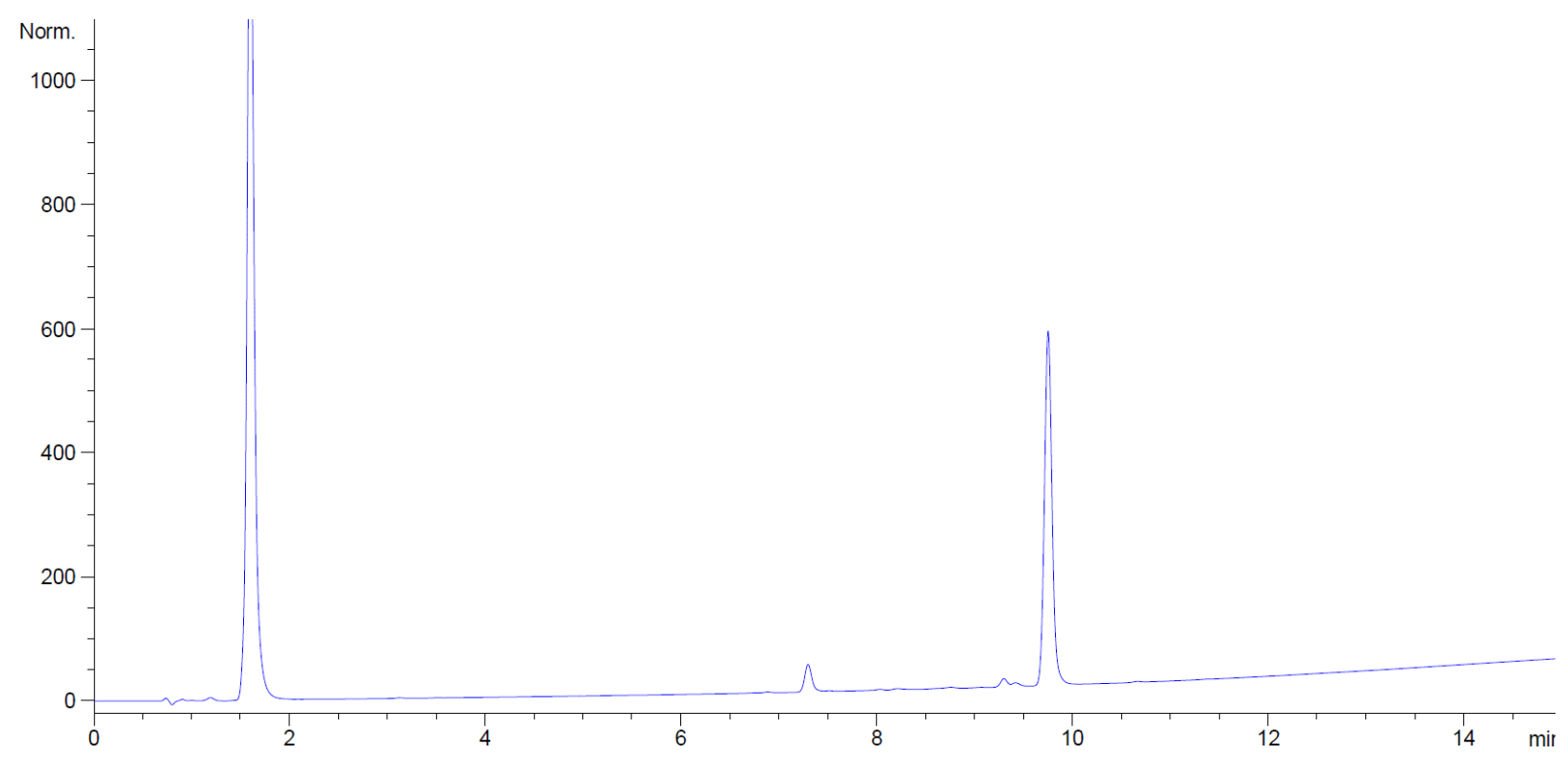

HPLC-S13. Fmoc-Arg(Pbf)-OH : DIC : OxymaPure [1:1.2:1], at $45^{\circ} \mathrm{C}$, in NBP, in presence of nucleophile (tripeptidyl resin), In-situ activation, $0.15 \mathrm{M}$. 
0 min

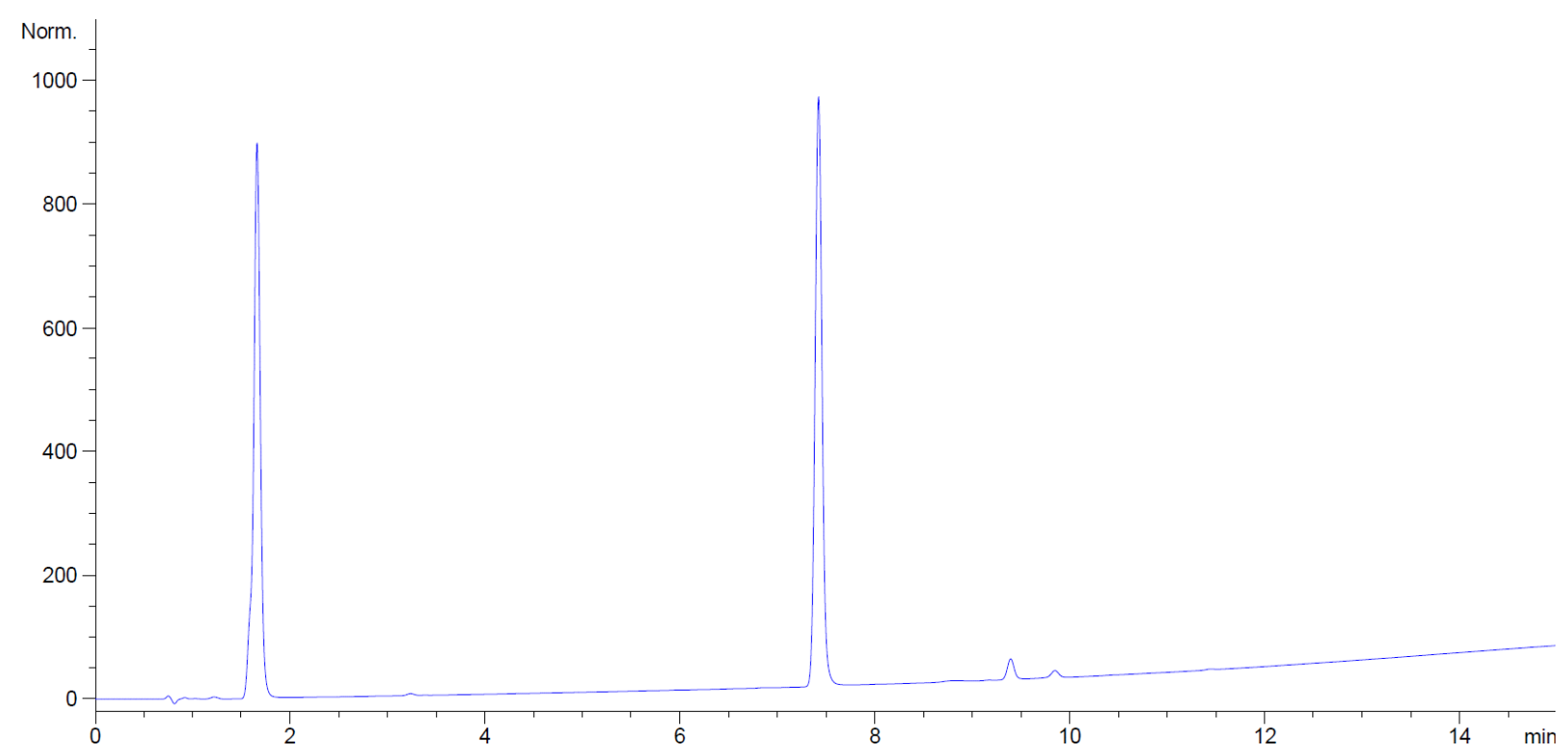

$30 \mathrm{~min}$

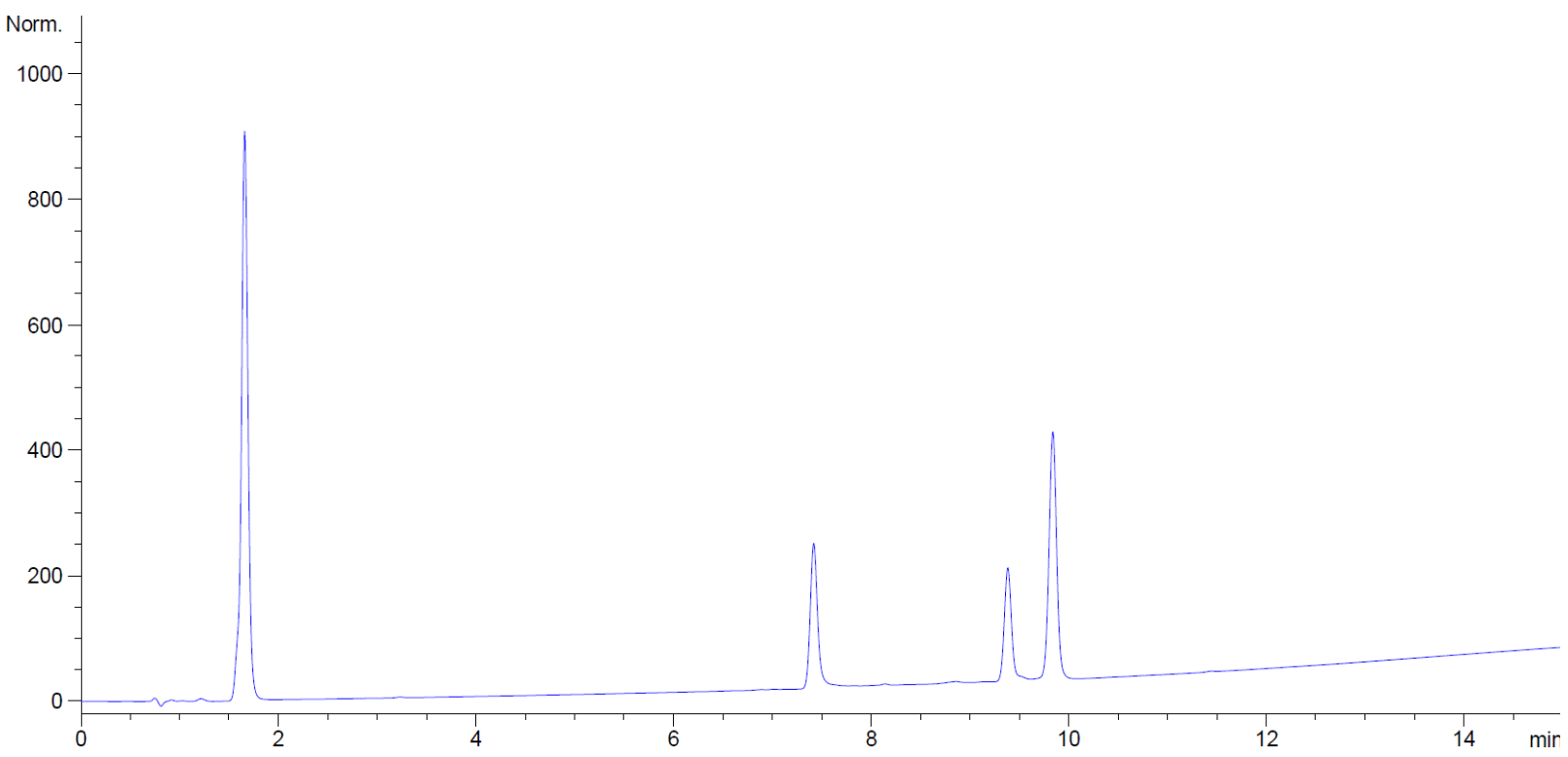

$60 \mathrm{~min}$ 


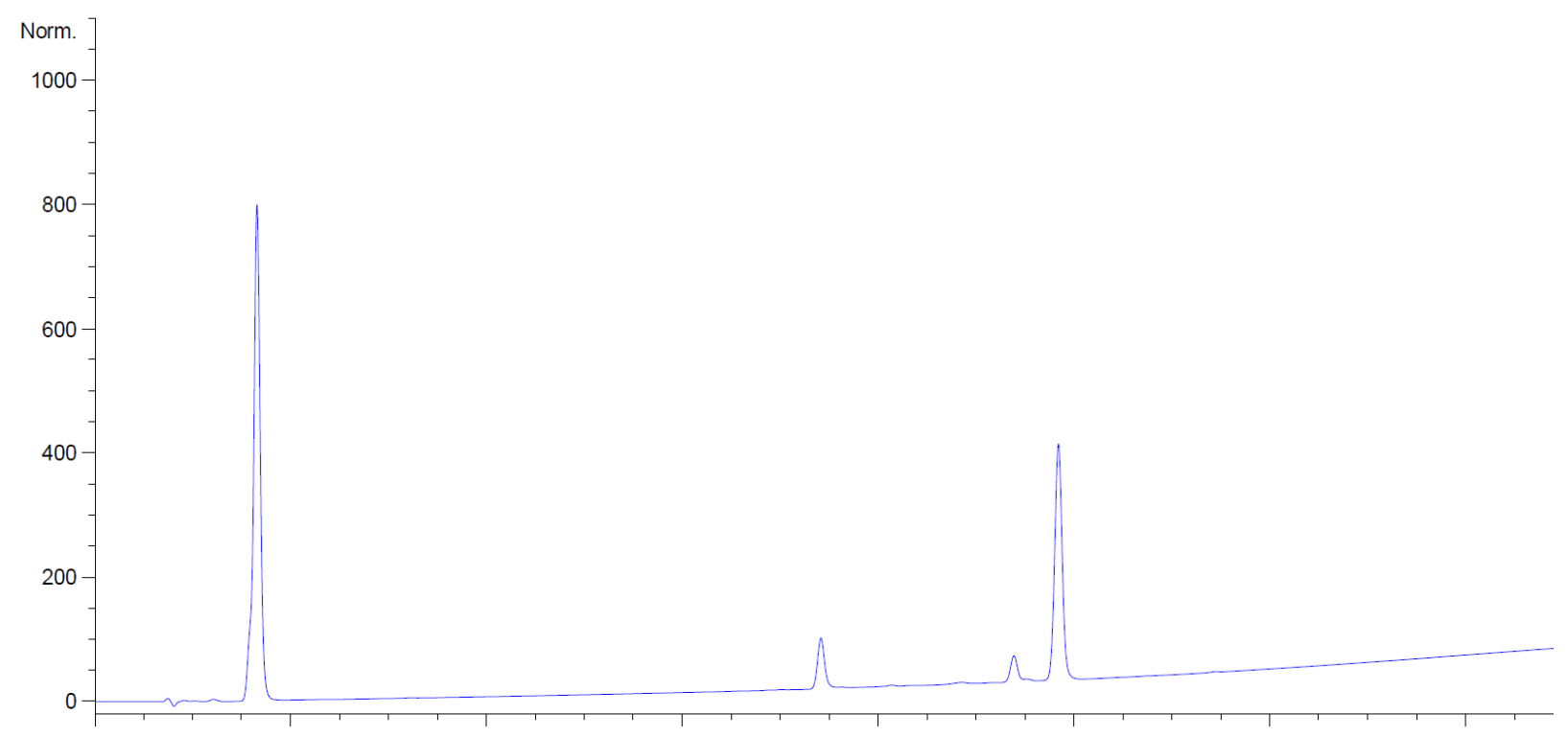

$120 \mathrm{~min}$

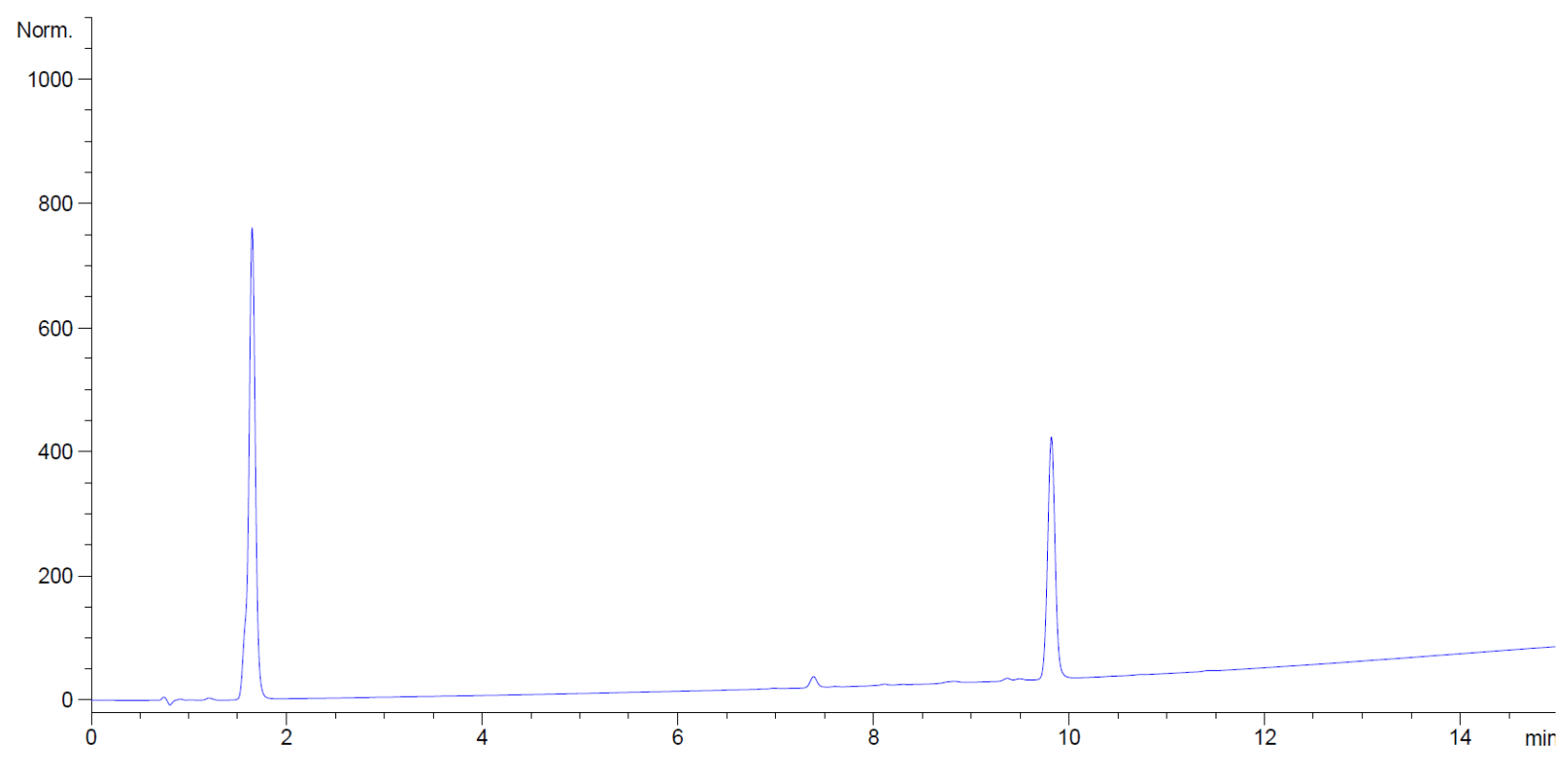

HPLC-S14. Fmoc-Arg(Pbf)-OH : DIC : OxymaPure [1:1.2:1], at $45^{\circ} \mathrm{C}$, in NBP, in presence of nucleophile (tripeptidyl resin), In-situ activation, half DIC at $0 \mathrm{~min}$ and half at $30 \mathrm{~min}, 0.15 \mathrm{M}$. 
0 min

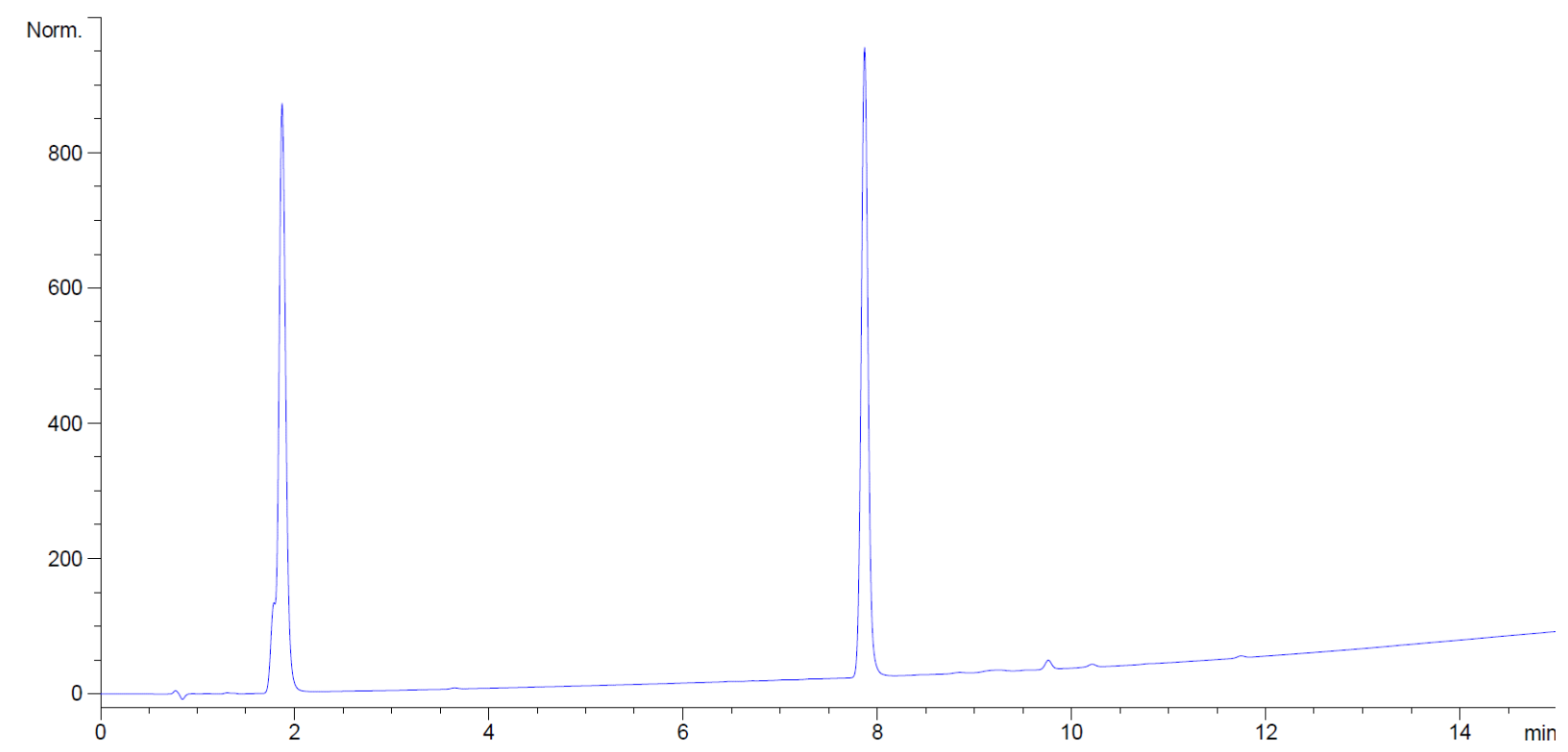

$30 \mathrm{~min}$

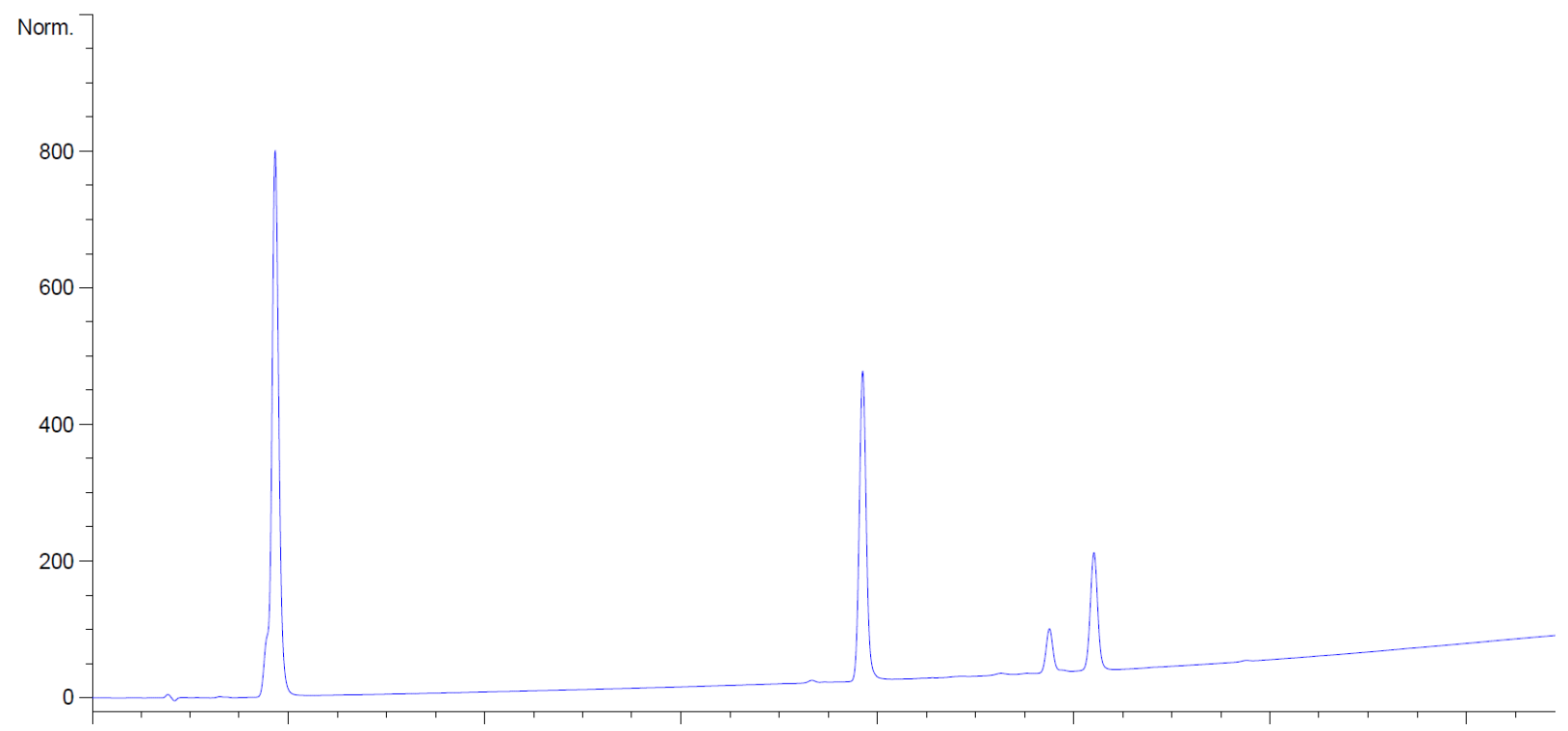

$60 \mathrm{~min}$ 


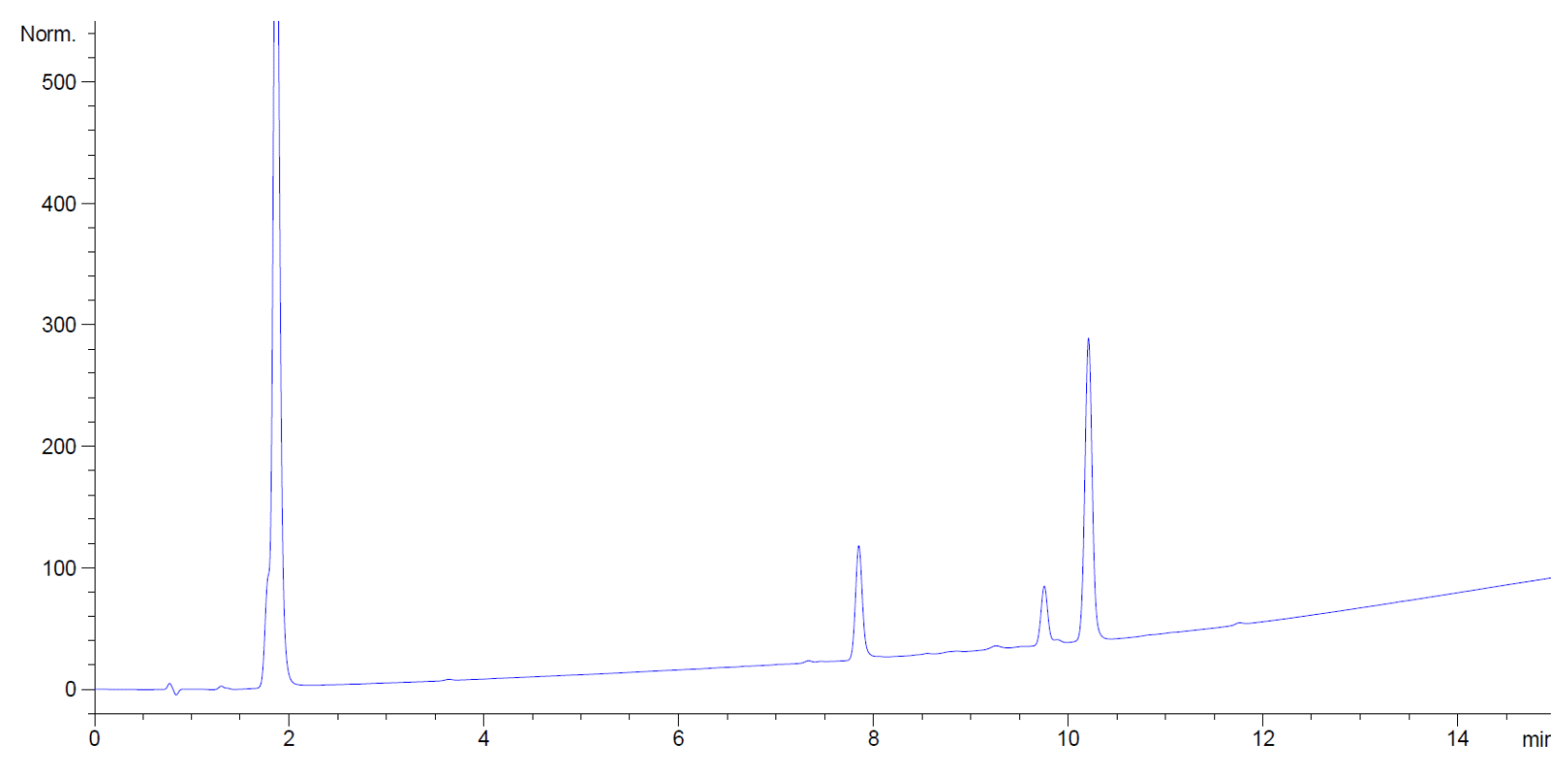

$120 \mathrm{~min}$

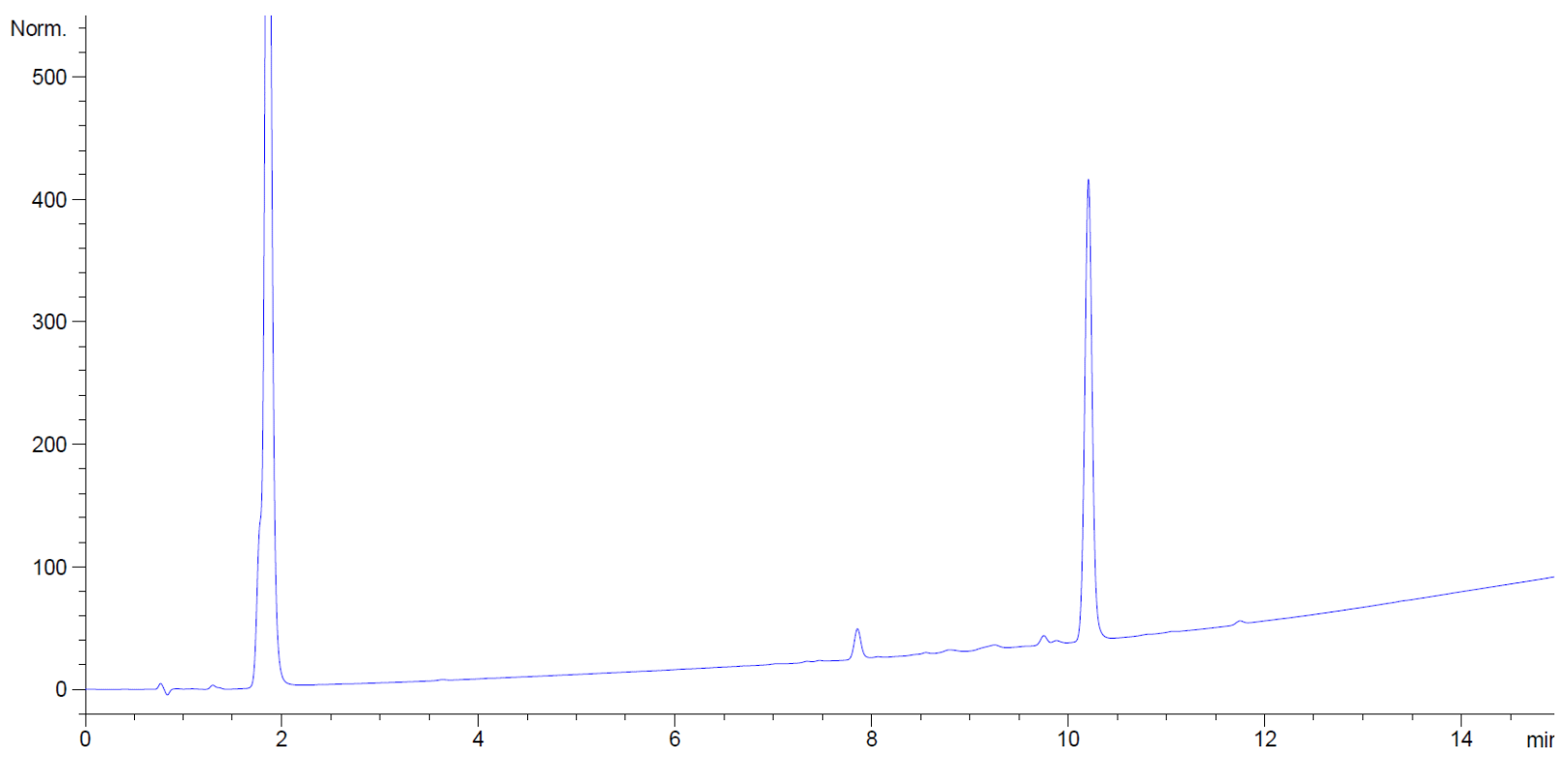

HPLC-S15. Fmoc-Arg(Pbf)-OH : DIC : OxymaPure [1:1.2:1], at $45^{\circ} \mathrm{C}$, in NBP, in presence of nucleophile (tripeptidyl resin), In-situ activation, half DIC at $0 \mathrm{~min}$ and half DIC +0.25 equiv. Fmoc-Arg(Pbf)-OH at $30 \mathrm{~min}, 0.15 \mathrm{M}$. 
0 min

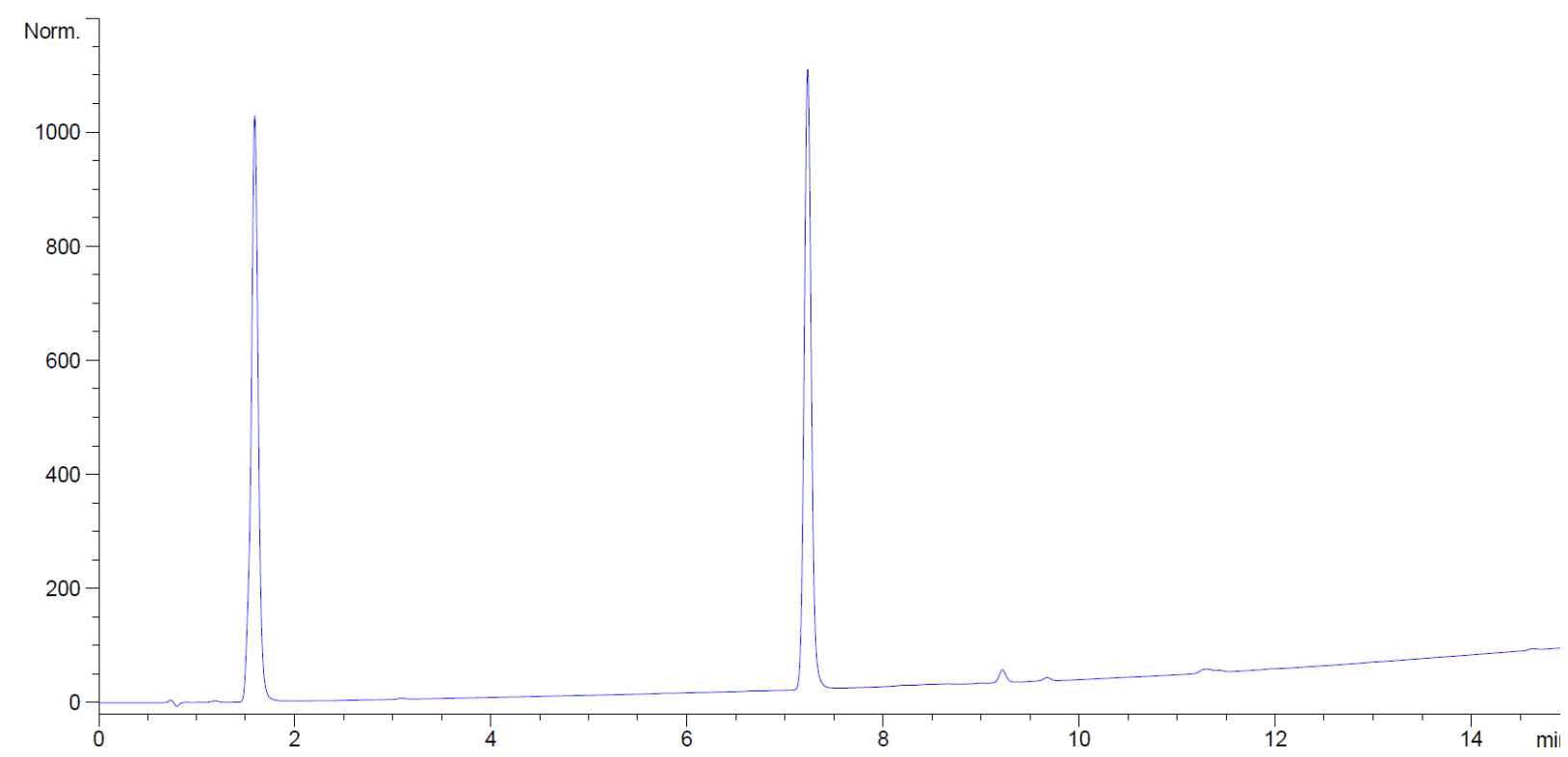

$30 \mathrm{~min}$

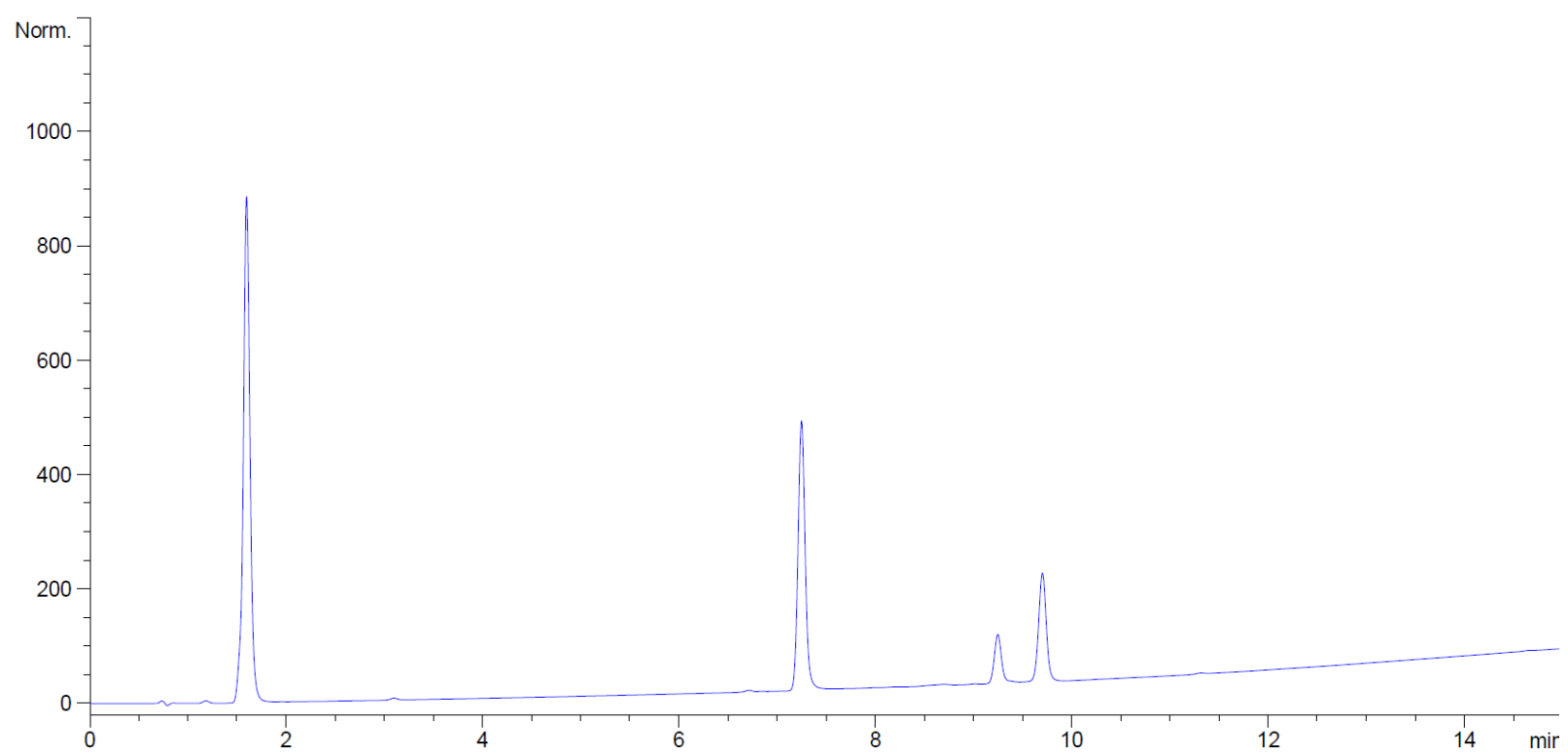

$60 \mathrm{~min}$ 


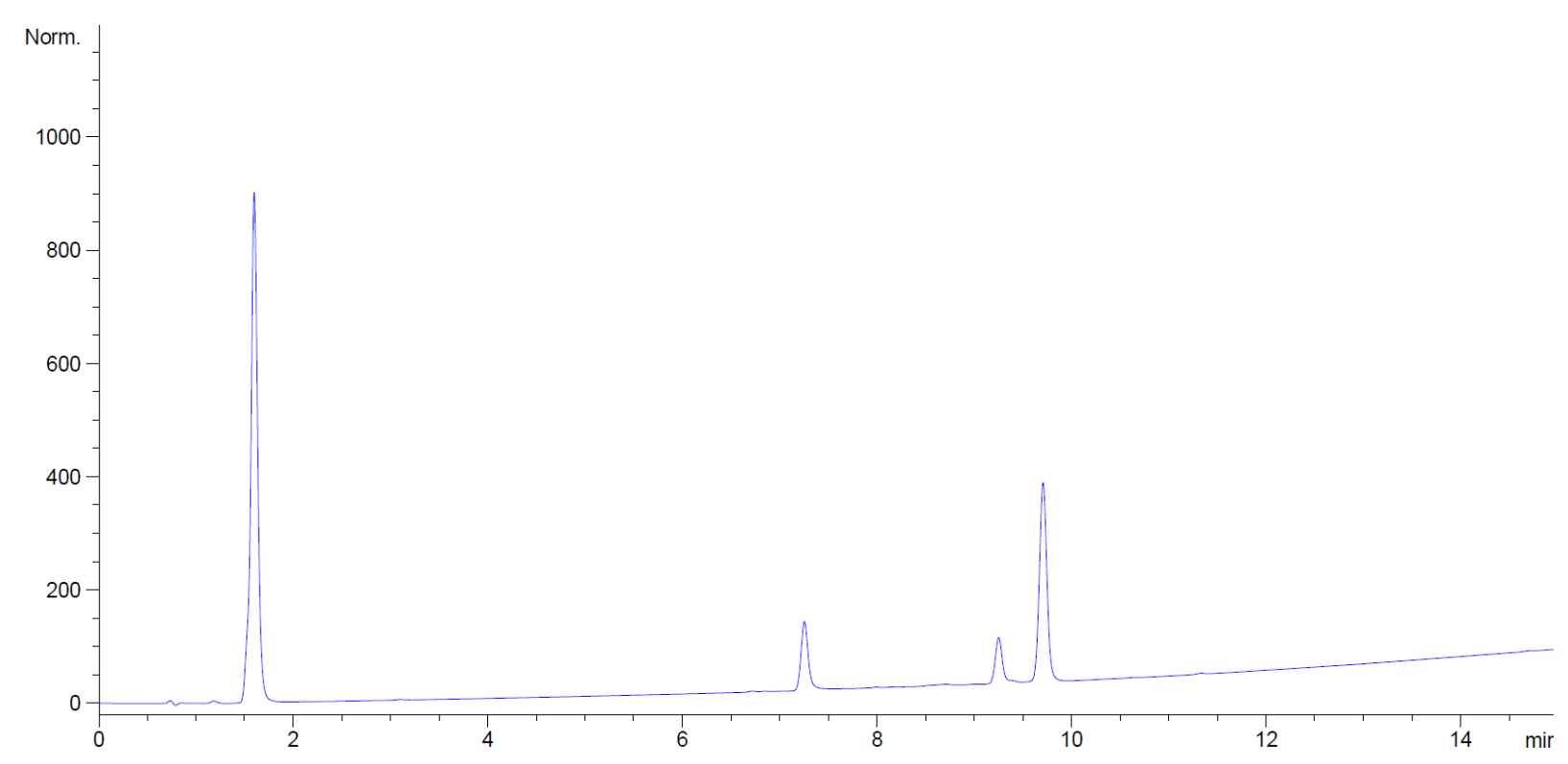

$120 \mathrm{~min}$

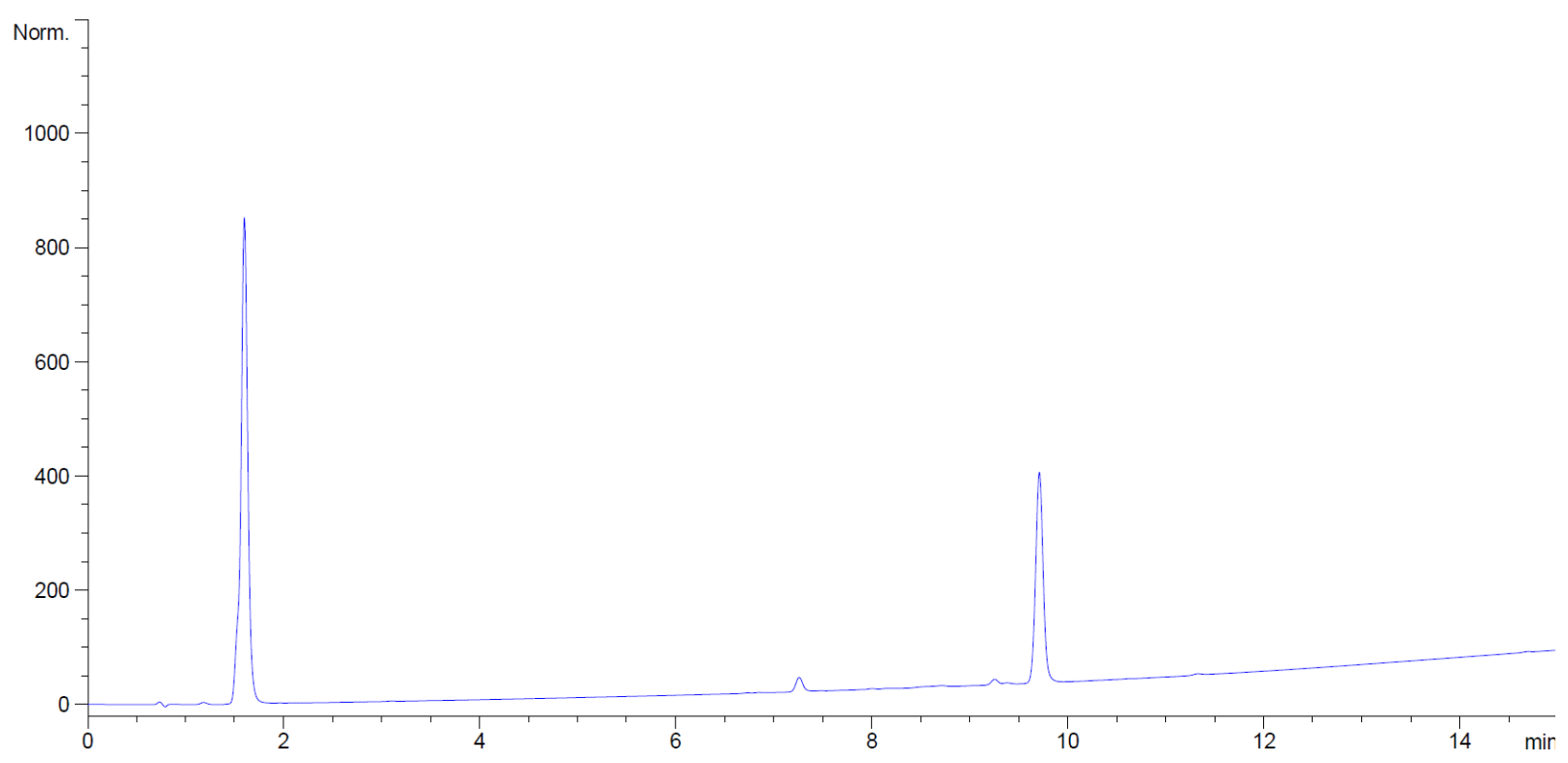

\section{Coupling quantification}

HPLC-S16. Coupling quantification of Tripeptide (H-GFL-NH $\left.\mathrm{N}_{2}\right)$ vs Tetrapeptide (H-RGFL$\mathrm{NH}_{2}$ ), Fmoc-Arg(Pbf)-OH : DIC : OxymaPure 1.5 eq. x [1:1:1], at RT, DMF. 


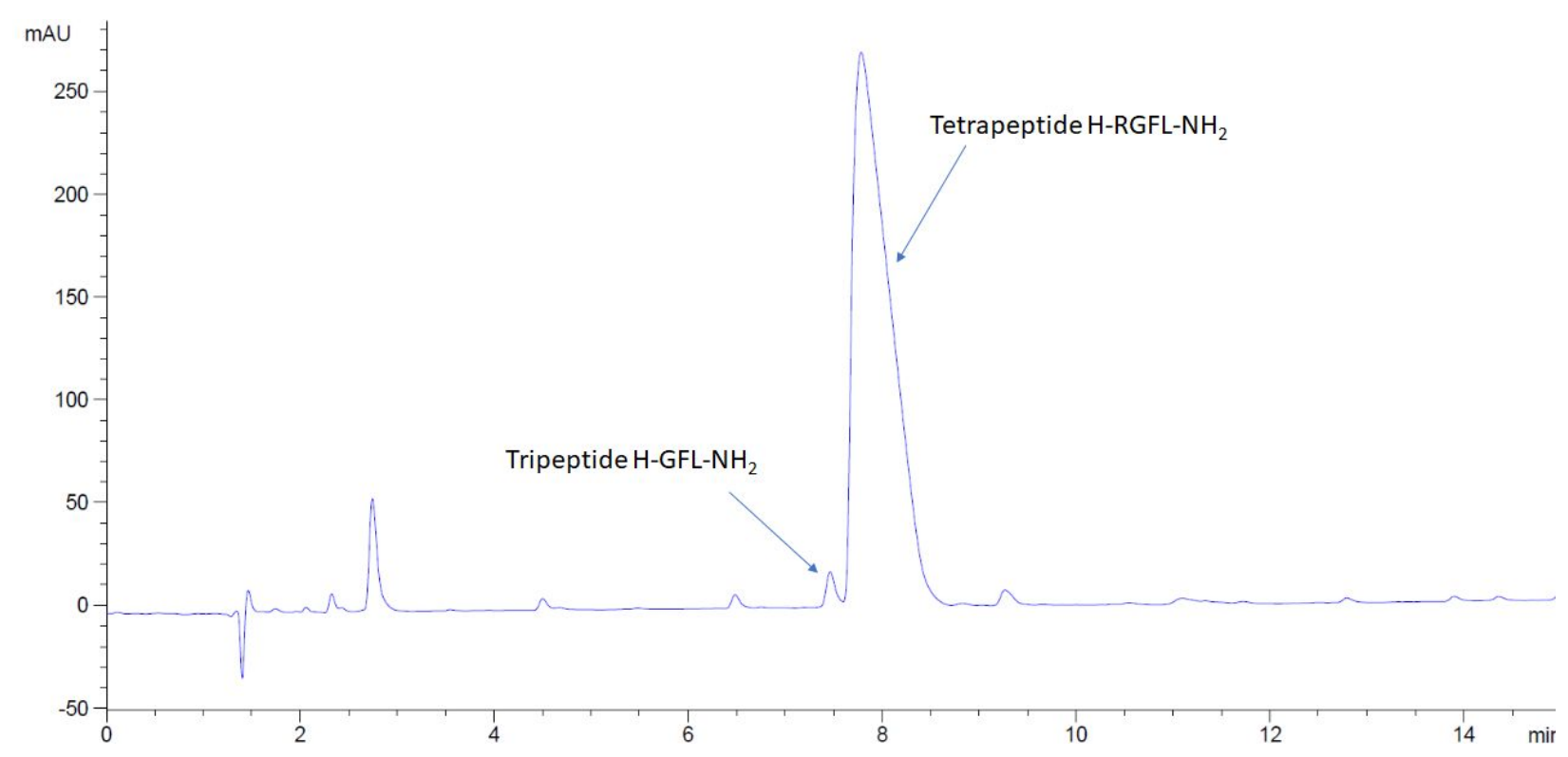

HPLC-S17. Coupling quantification of Tripeptide $\left(\mathrm{H}-\mathrm{GFL}-\mathrm{NH}_{2}\right)$ vs Tetrapeptide (H-RGFL$\mathrm{NH}_{2}$ ), Fmoc-Arg(Pbf)-OH : DIC : OxymaPure 1.5 eq. $\mathrm{x}[1: 1: 1], 45^{\circ} \mathrm{C}$, DMF.

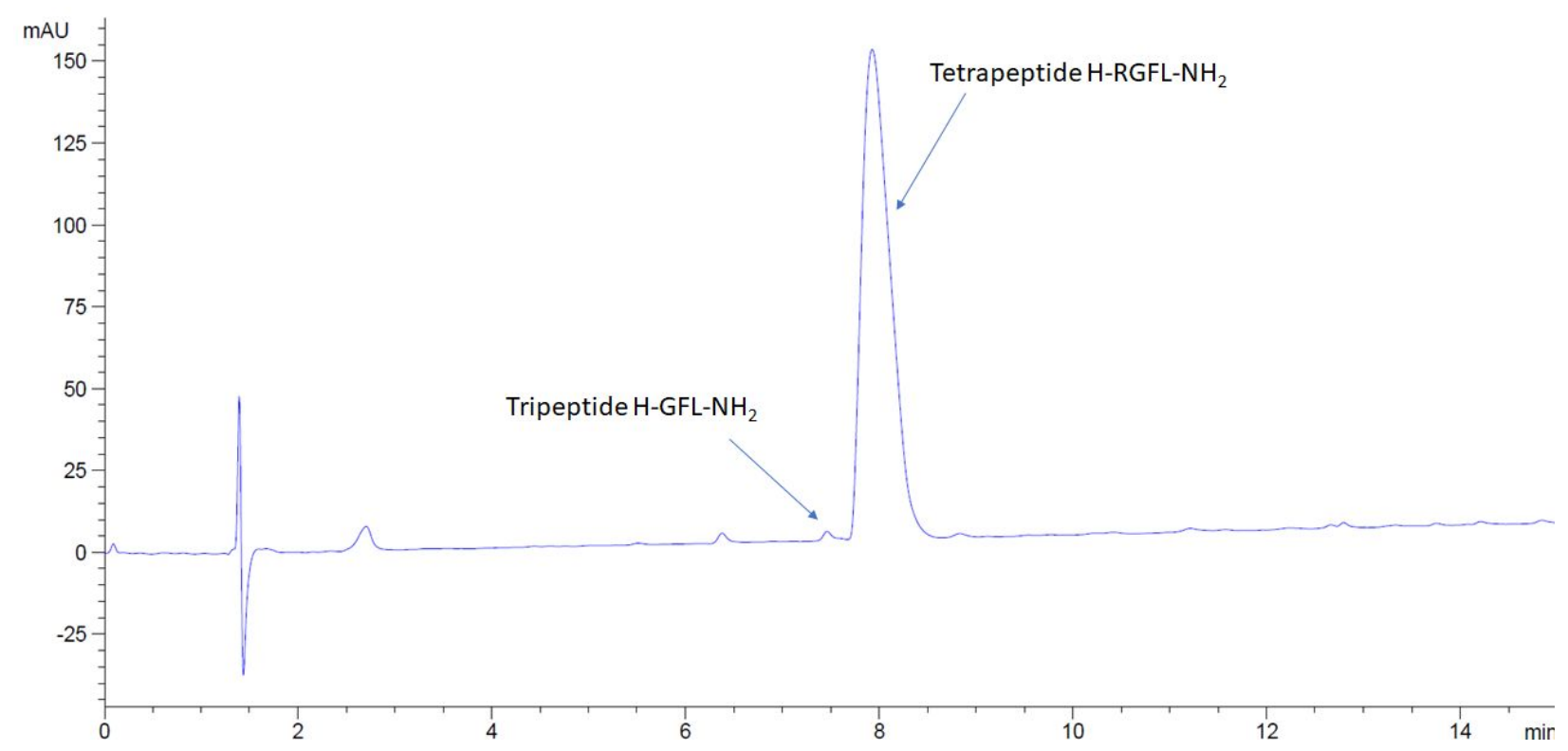

HPLC-S18. Coupling quantification of Tripeptide $\left(\mathrm{H}-\mathrm{GFL}-\mathrm{NH}_{2}\right)$ vs Tetrapeptide (H-RGFL$\mathrm{NH}_{2}$ ), Fmoc-Arg(Pbf)-OH : DIC : OxymaPure 1.5 eq. x [1:1:1], RT, NBP. 


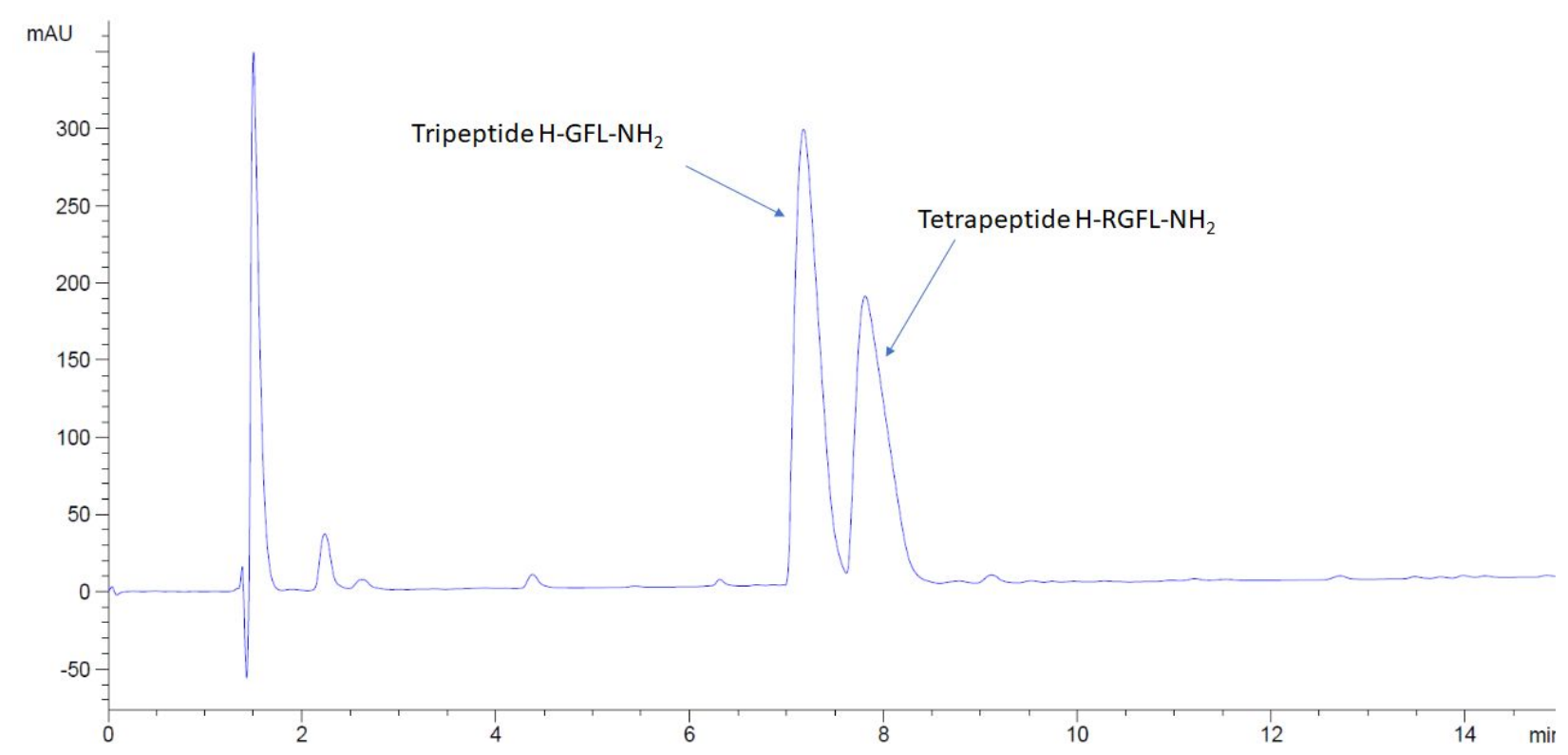

S19. Coupling quantification of Tripeptide $\left(\mathrm{H}-\mathrm{GFL}-\mathrm{NH}_{2}\right)$ vs Tetrapeptide $\left(\mathrm{H}-\mathrm{RGFL}-\mathrm{NH}_{2}\right)$, Fmoc-Arg(Pbf)-OH : DIC : OxymaPure 1.5 eq. $\mathrm{x}[1: 1: 1], 45^{\circ} \mathrm{C}, \mathrm{NBP}$.

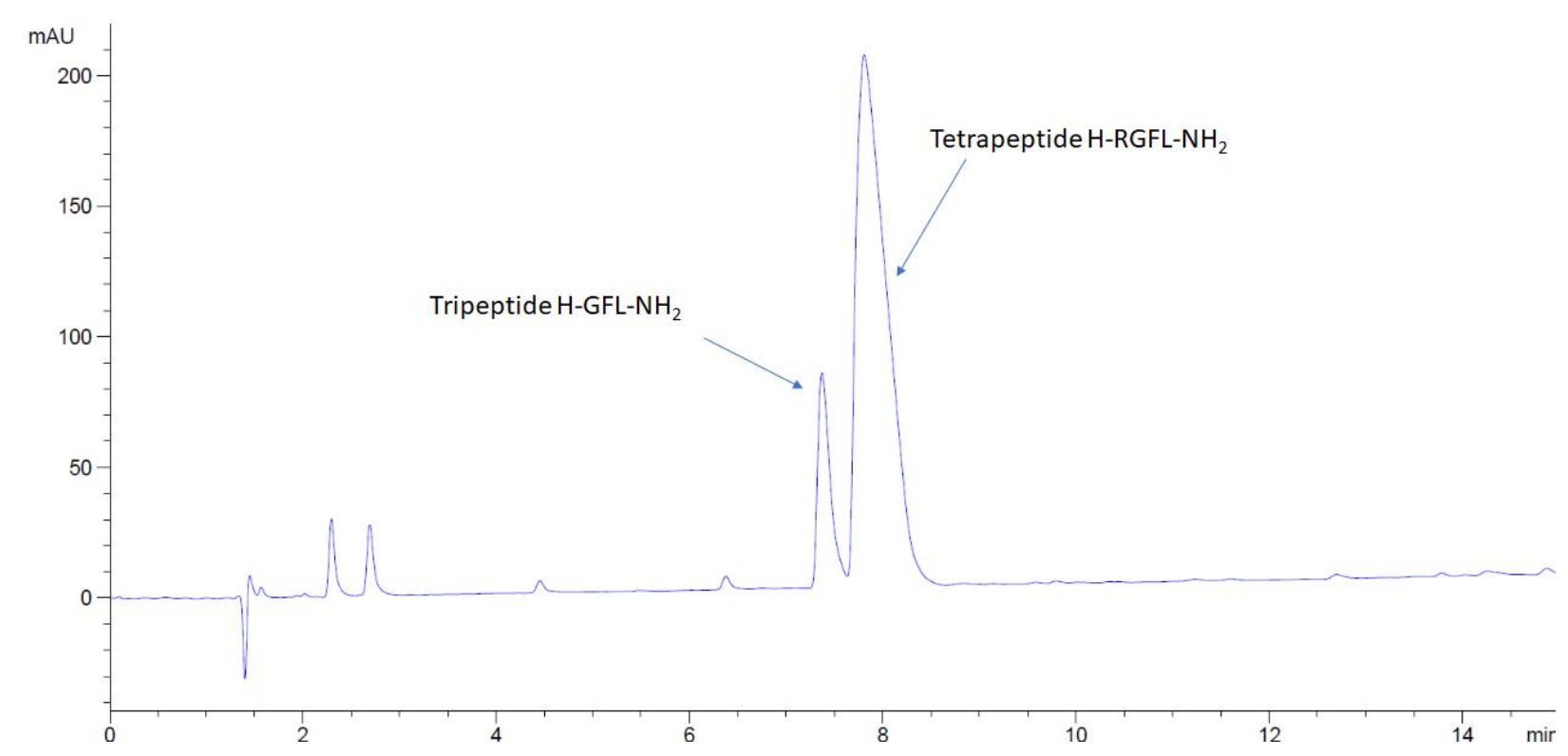

HPLC-S20. Coupling quantification of Tripeptide $\left(\mathrm{H}-\mathrm{GFL}-\mathrm{NH}_{2}\right)$ vs Tetrapeptide (H-RGFL$\mathrm{NH}_{2}$ ), Fmoc-Arg(Pbf)-OH : DIC : OxymaPure 1.5 eq. x [1:1:1], $60^{\circ} \mathrm{C}, \mathrm{NBP}$. 


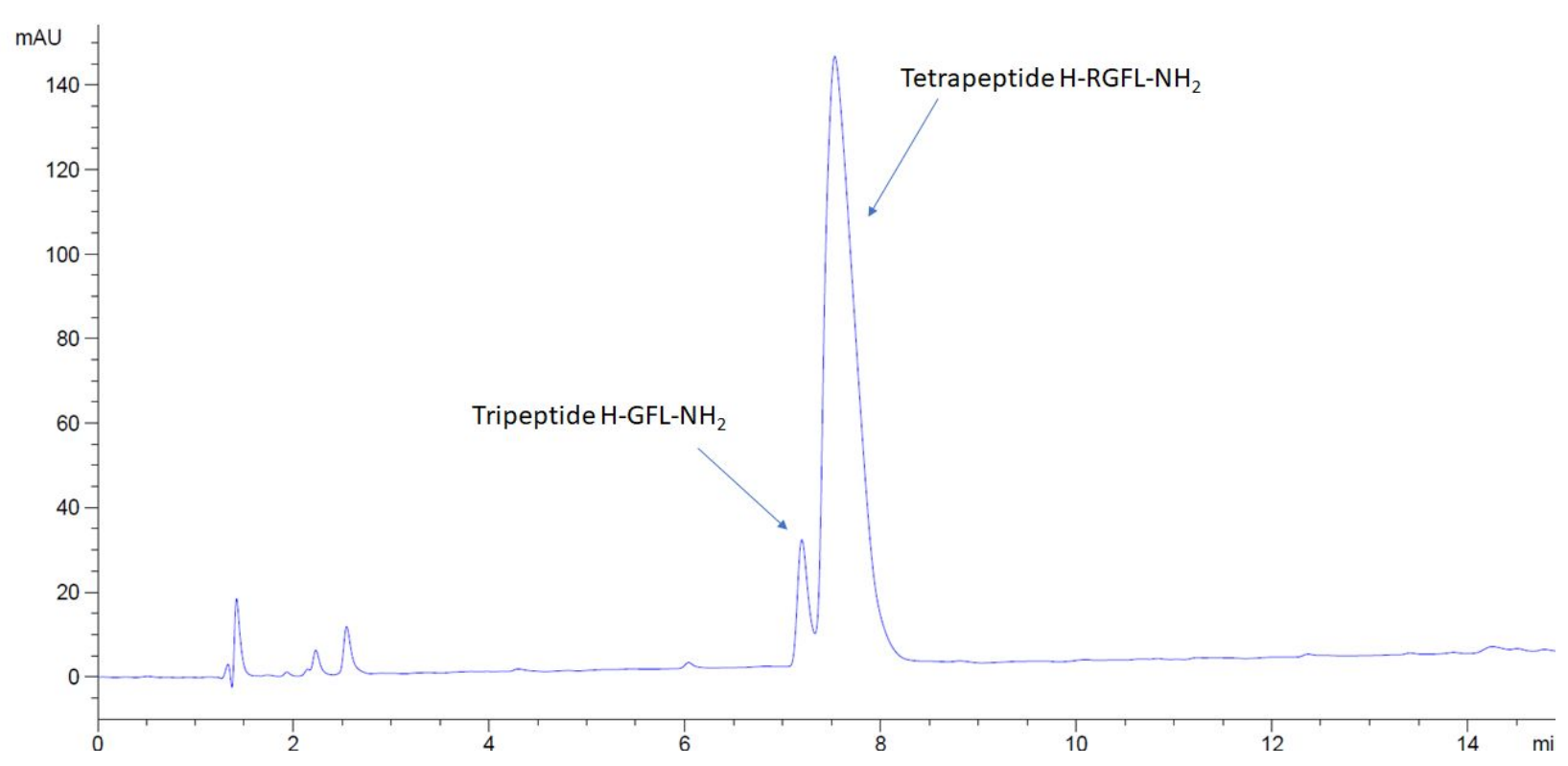

HPLC-S21. Coupling quantification of Tripeptide (H-GFL-NH $\left.\mathrm{N}_{2}\right)$ vs Tetrapeptide (H-RGFL$\mathrm{NH}_{2}$ ), Fmoc-Arg(Pbf)-OH : DIC : OxymaPure 1.5 eq. x [1:1:1], $45^{\circ} \mathrm{C}$, NBP, Pre-conditioning strategy.

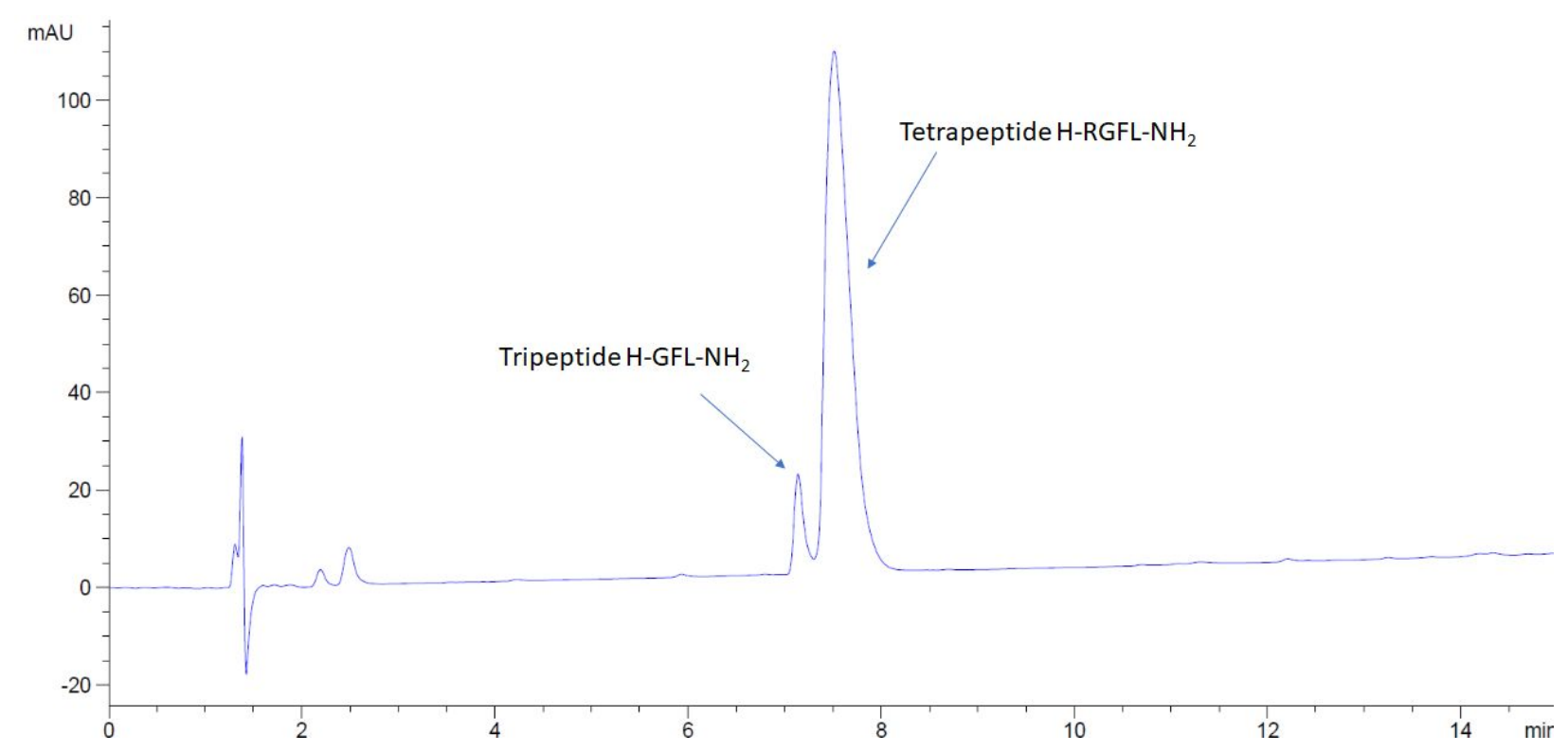

HPLC-S22. Coupling quantification of Tripeptide (H-GFL-NH $\mathrm{N}_{2}$ ) $s$ Tetrapeptide (H-RGFL$\mathrm{NH}_{2}$ ), Fmoc-Arg(Pbf)-OH : DIC : OxymaPure 1.5 eq. x [1:1:1], $45^{\circ} \mathrm{C}, \mathrm{NBP}$, In situ Activation, $0.15 \mathrm{M}$. 


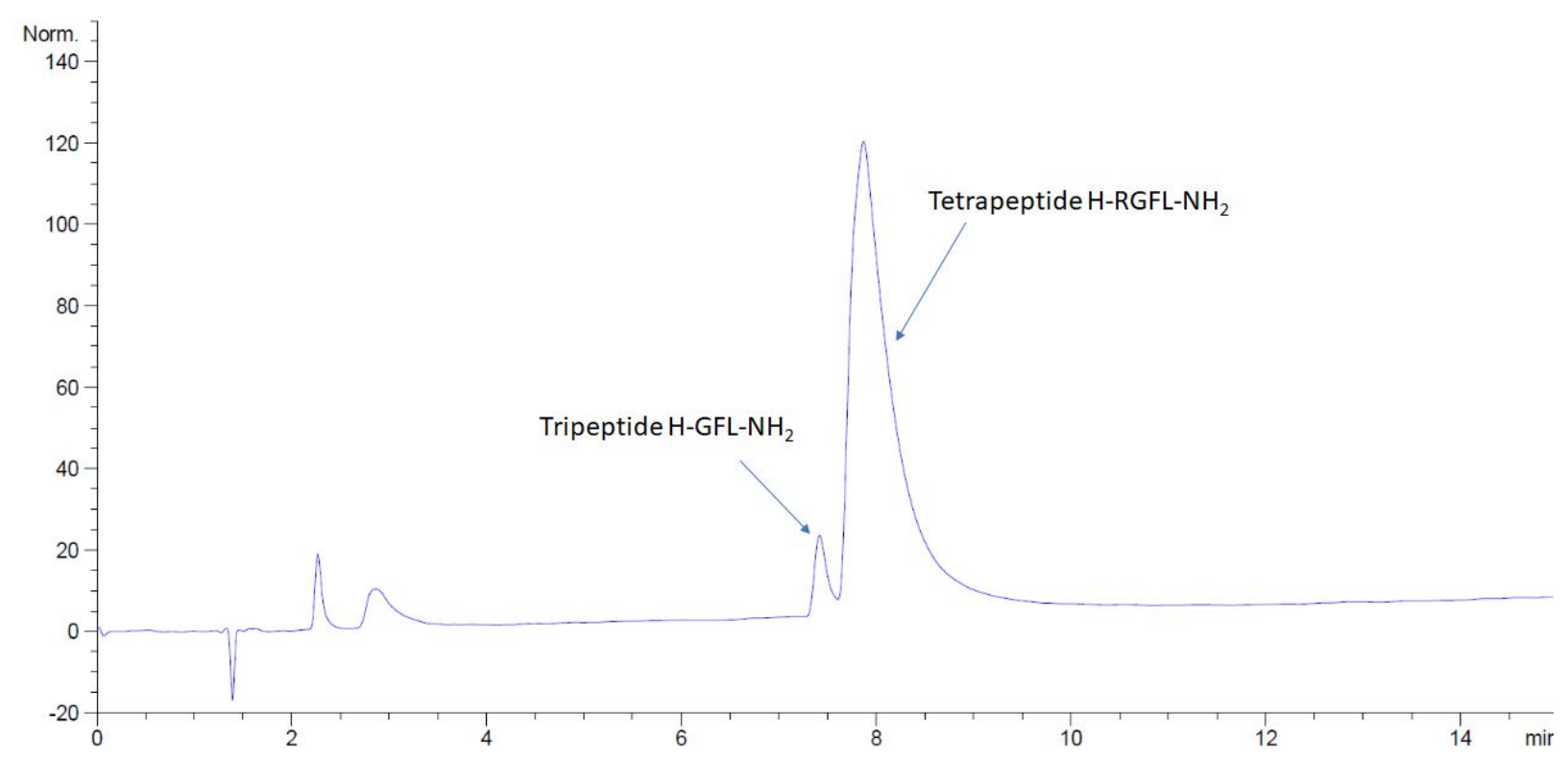

HPLC-S23. Coupling quantification of Tripeptide $\left(\mathrm{H}-\mathrm{GFL}-\mathrm{NH}_{2}\right)$ vs Tetrapeptide (H-RGFL$\mathrm{NH}_{2}$ ), Fmoc-Arg(Pbf)-OH : DIC : OxymaPure 1.5 eq. $\mathrm{x}[1: 1: 1], 45^{\circ} \mathrm{C}, \mathrm{NBP}$, In situ Activation, $0.075 \mathrm{M}$.

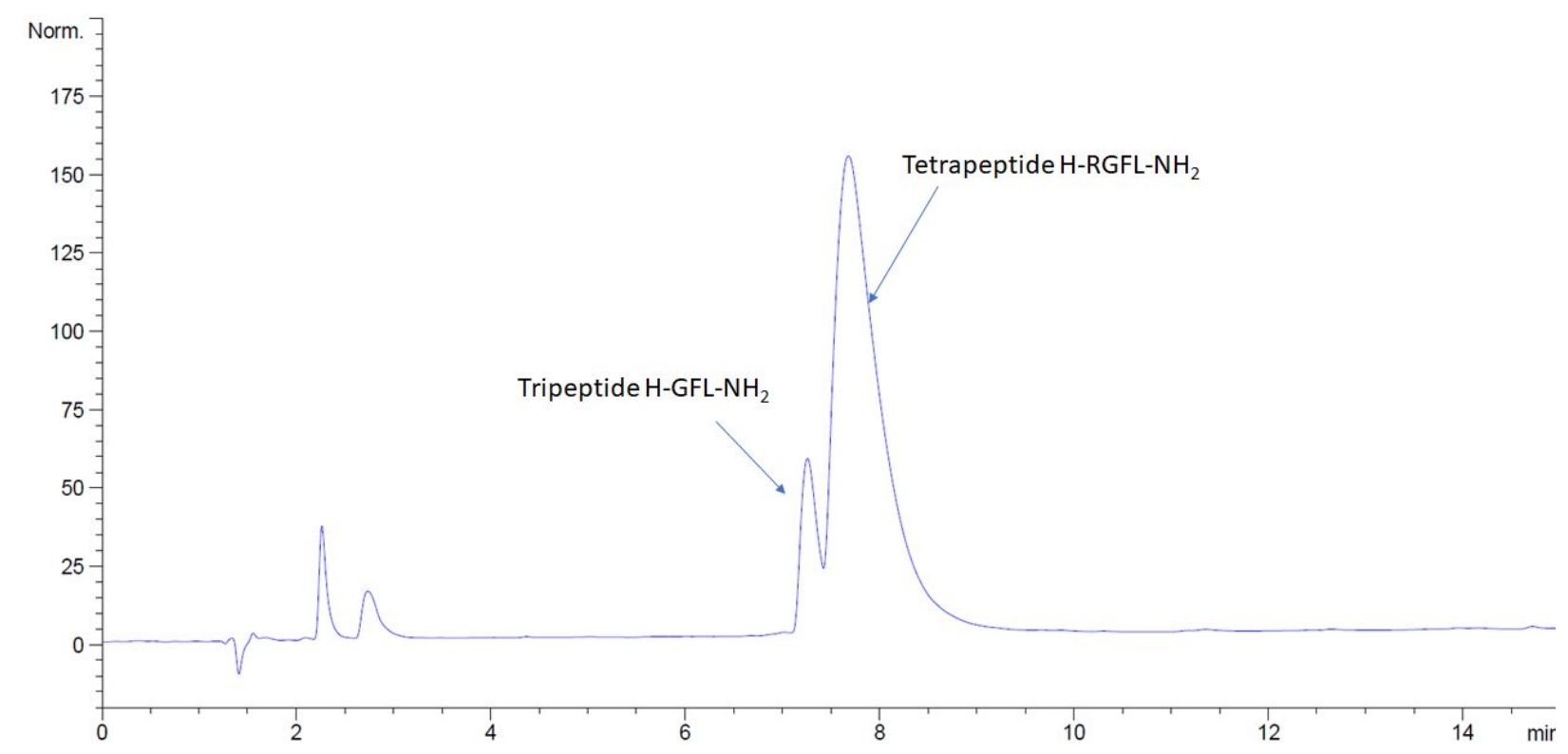

HPLC-S24. Coupling quantification of Tripeptide $\left(\mathrm{H}-\mathrm{GFL}-\mathrm{NH}_{2}\right)$ vs Tetrapeptide (H-RGFL$\mathrm{NH}_{2}$ ), Fmoc-Arg(Pbf)-OH : DIC : OxymaPure 1.5 eq. x [1:1.2:1], $45^{\circ} \mathrm{C}, \mathrm{NBP}$, In situ Activation, $0.075 \mathrm{M}$, half DIC at $0 \mathrm{~min}+$ half DIC and 0.25 eq Fmoc-Arf(Pbf)-OH at $30 \mathrm{~min}$. 


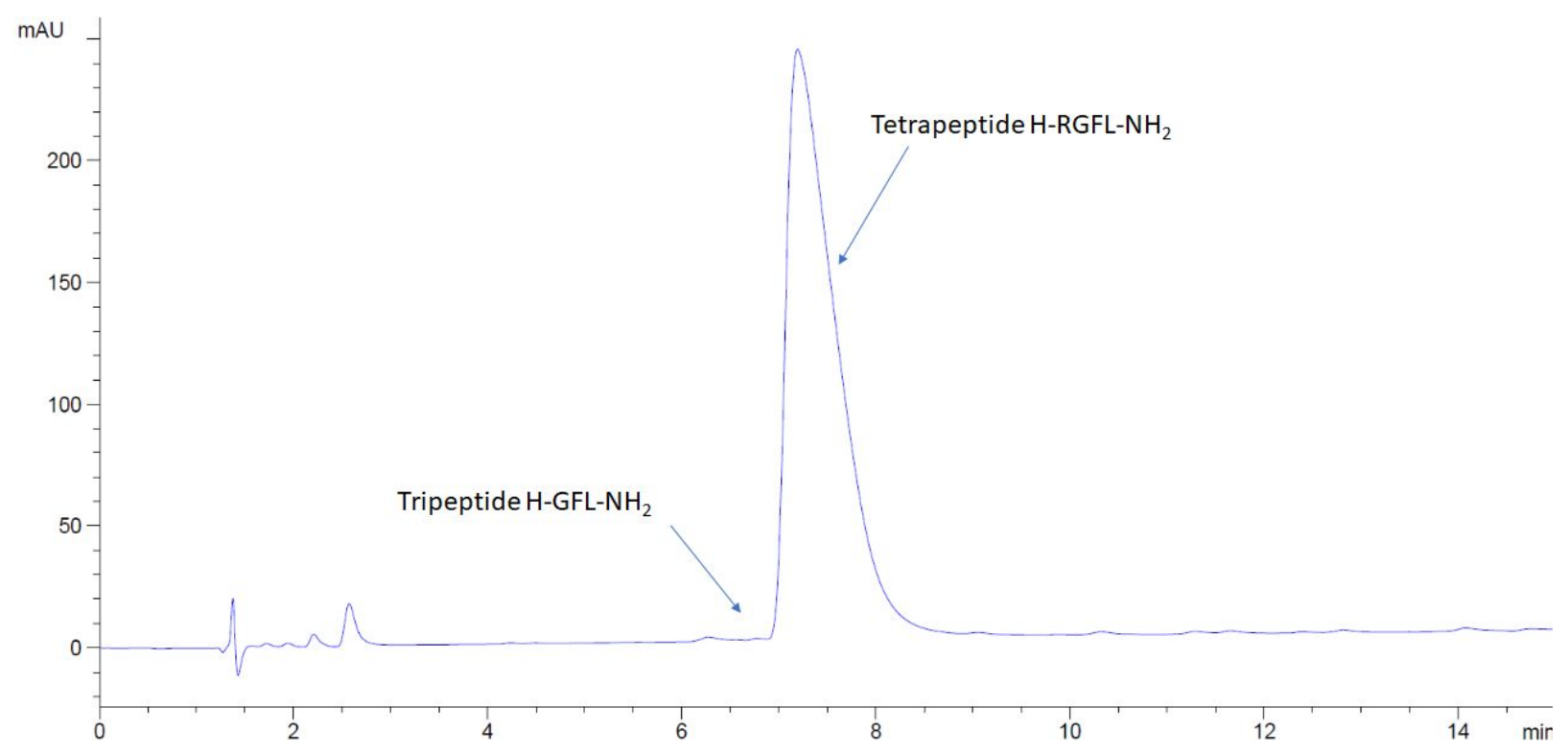

HPLC-S25. SPPS of Linear precursor of RGD Peptide (H-DfKRG-OH) in DMF

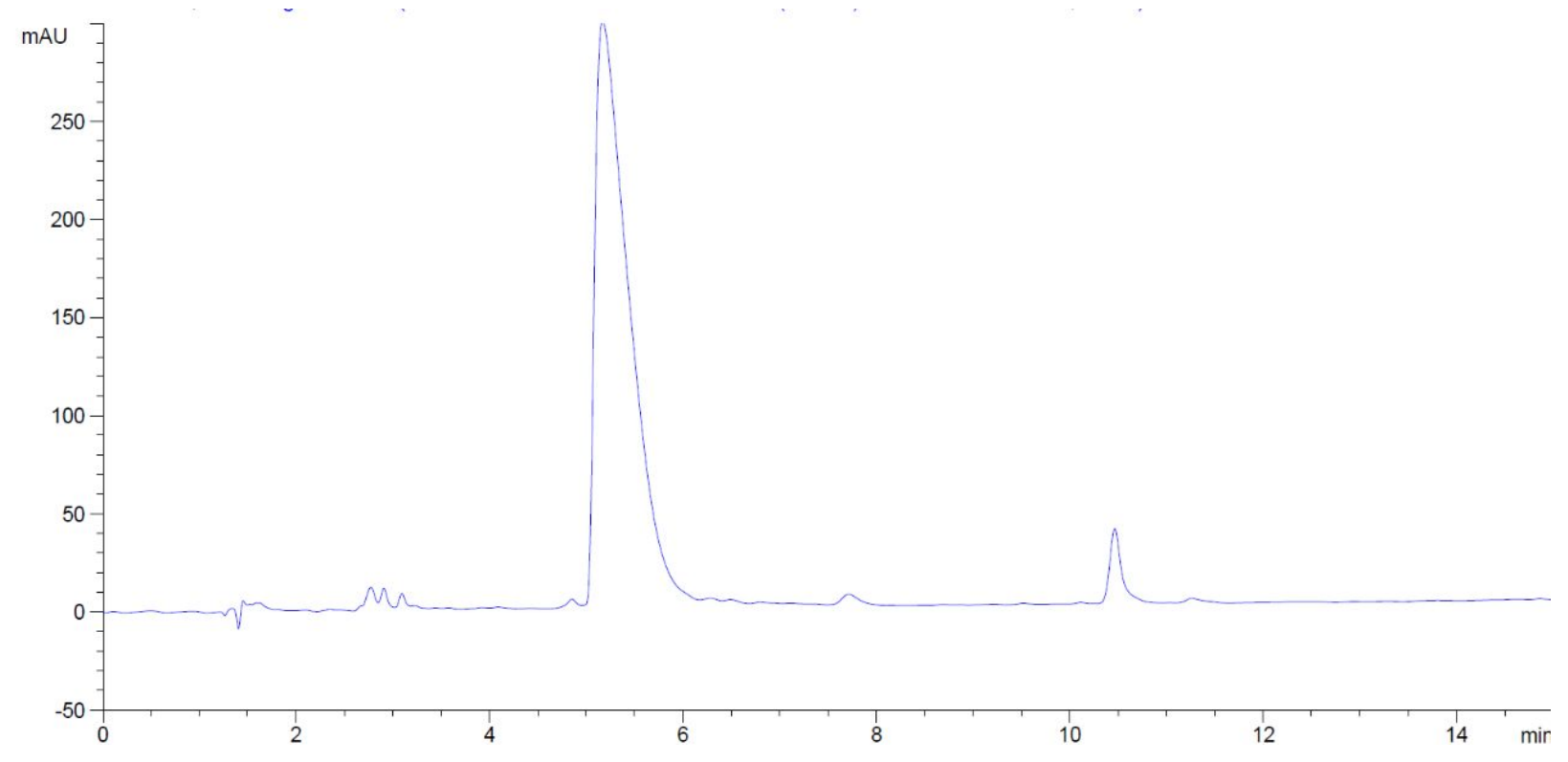

HPLC-S26. SPPS of Linear precursor of RGD Peptide (H-DfKRG-OH) in NBP 


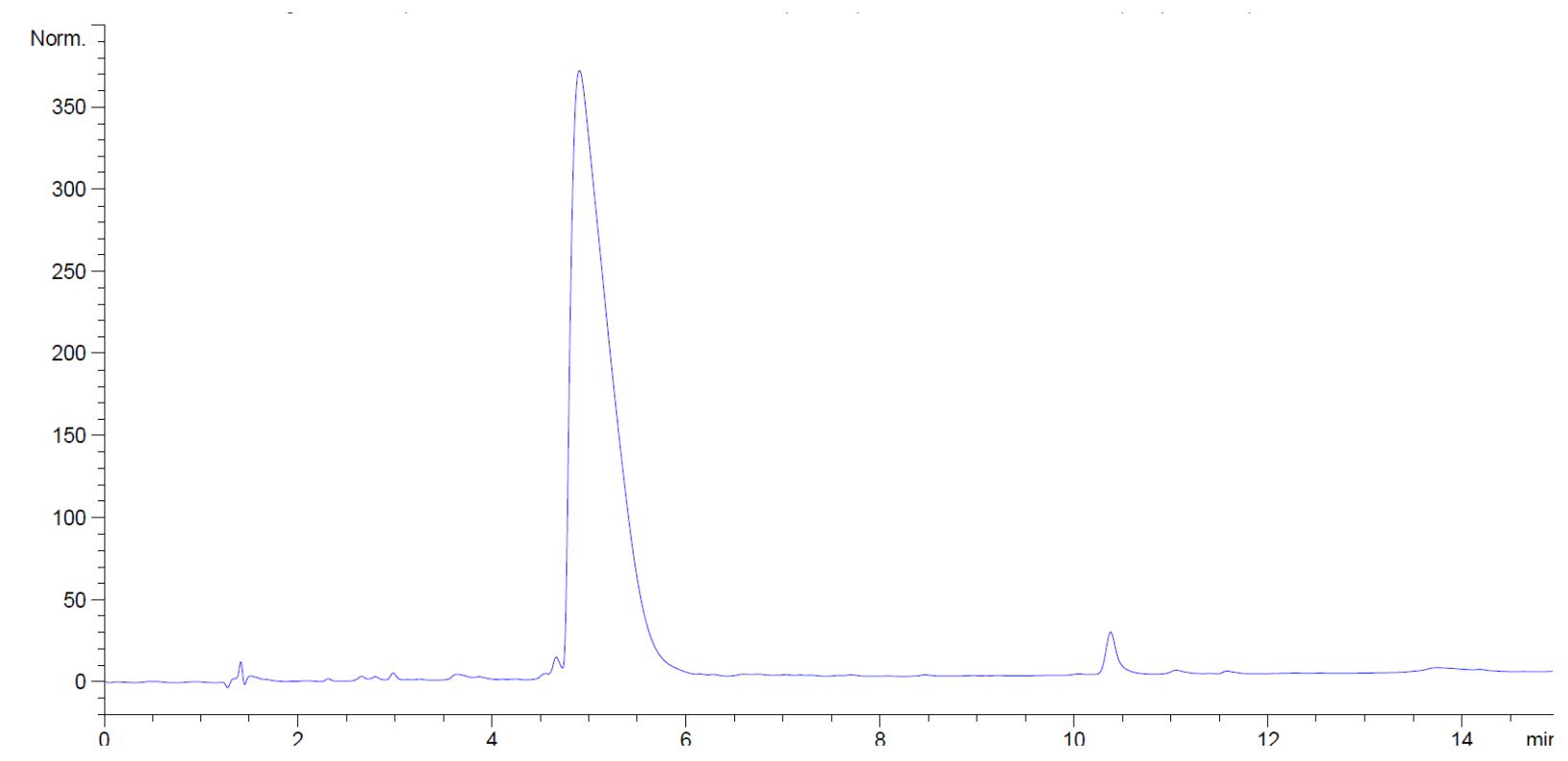

HPLC-S27. Des-Arg Peptide (H-DfKG-OH) in DMF

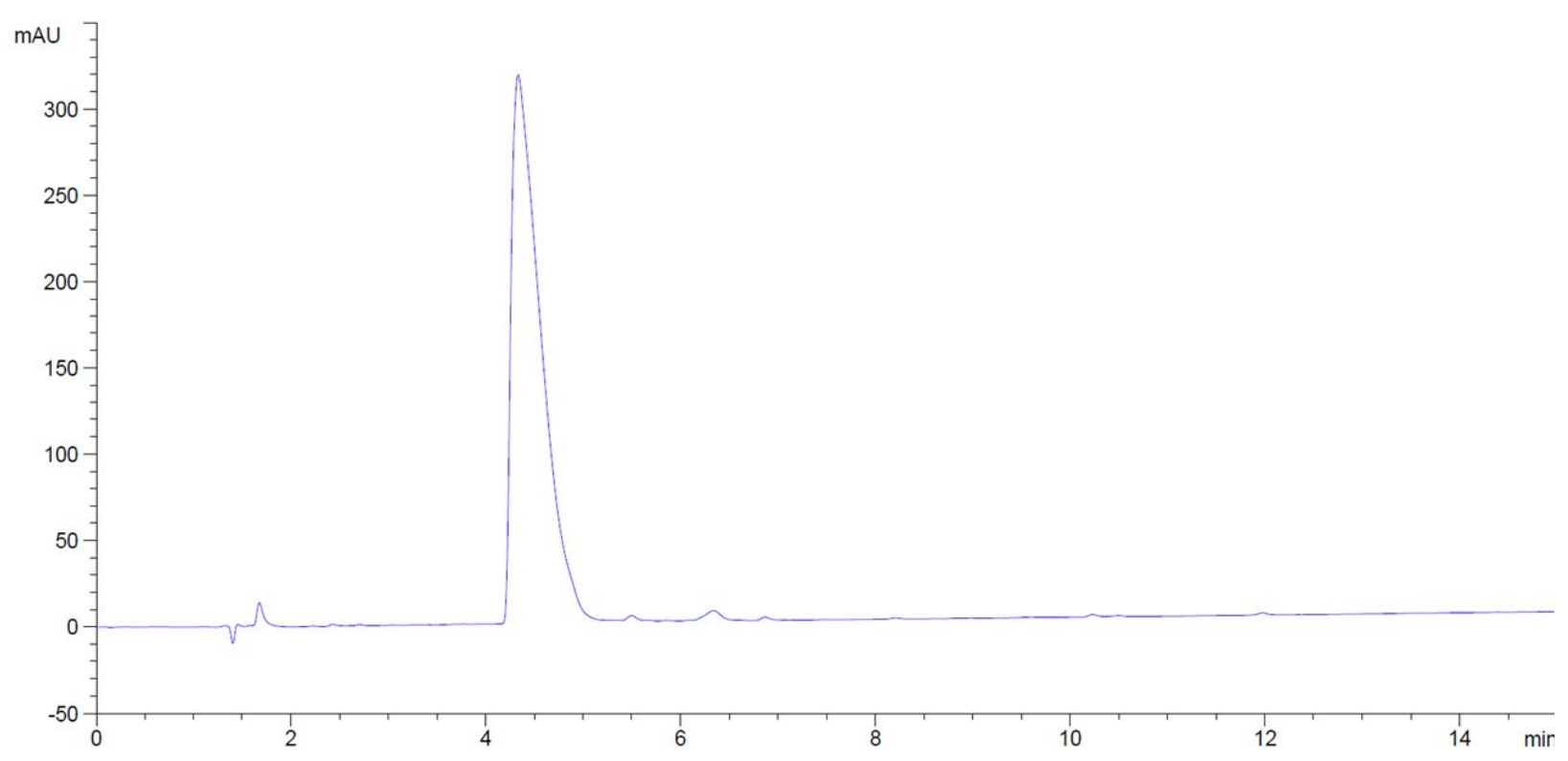

